\title{
Dynamic cerebral autoregulation : from methodology towards clinical application
}

Citation for published version (APA):

Gommer, E. D. (2013). Dynamic cerebral autoregulation : from methodology towards clinical application. [Doctoral Thesis, Maastricht University]. Maastricht University. https://doi.org/10.26481/dis.20130405eg

Document status and date:

Published: 01/01/2013

DOI:

10.26481/dis.20130405eg

Document Version:

Publisher's PDF, also known as Version of record

\section{Please check the document version of this publication:}

- A submitted manuscript is the version of the article upon submission and before peer-review. There can be important differences between the submitted version and the official published version of record.

People interested in the research are advised to contact the author for the final version of the publication, or visit the DOI to the publisher's website.

- The final author version and the galley proof are versions of the publication after peer review.

- The final published version features the final layout of the paper including the volume, issue and page numbers.

Link to publication

\footnotetext{
General rights rights.

- You may freely distribute the URL identifying the publication in the public portal. please follow below link for the End User Agreement:

www.umlib.nl/taverne-license

Take down policy

If you believe that this document breaches copyright please contact us at:

repository@maastrichtuniversity.nl

providing details and we will investigate your claim.
}

Copyright and moral rights for the publications made accessible in the public portal are retained by the authors and/or other copyright owners and it is a condition of accessing publications that users recognise and abide by the legal requirements associated with these

- Users may download and print one copy of any publication from the public portal for the purpose of private study or research.

- You may not further distribute the material or use it for any profit-making activity or commercial gain

If the publication is distributed under the terms of Article $25 \mathrm{fa}$ of the Dutch Copyright Act, indicated by the "Taverne" license above, 


\section{Dynamic Cerebral Autoregulation:}

from methodology towards clinical application

\section{PROEFSCHRIFT}

ter verkrijging van de graad van doctor

aan de Universiteit Maastricht, op gezag van de Rector Magnificus,

Prof. dr. L.L.G. Soete

volgens het besluit van het College van Decanen,

in het openbaar te verdedigen

op vrijdag 5 april 2013 om 14.00 uur

door

Erik Dirk Gommer

geboren op 10 februari 1968 te Emmen 
Promotores:

Prof. dr. W.H. Mess

Prof. dr. R.B. Panerai (University of Leicester, United Kingdom)

Copromotor:

Dr. ir. J.P.H. Reulen

Beoordelingscommissie:

Prof. dr. ir. F.N. van de Vosse (voorzitter)

Dr. J.A.H.R. Claassen (UMC St. Radboud Nijmegen)

Prof. dr. J.G. van Dijk (Leids UMC)

Prof. dr. H. Kingma

Prof. dr. M.J. Post 


\section{Contents}

$\begin{array}{lll}\text { Chapter } 1 & \text { Introduction } & 7\end{array}$

Chapter $2 \quad$ Dynamic cerebral autoregulation: different signal

19 processing methods without influence on results and reproducibility

Chapter 3 Dynamic cerebral autoregulation and cerebrovascular reactivity: a comparative study in lacunar infarct patients

Chapter 4 Dynamic Cerebral Autoregulation in Subjects with Alzheimer's Disease, Mild Cognitive Impairment, and Controls: Evidence for Increased Peripheral Vascular Resistance with Possible Predictive Value

Chapter 5

Visually evoked blood flow responses and interaction with dynamic cerebral autoregulation: correction for blood pressure variation

Chapter 6

Dynamic assessment of cerebral autoregulation and cerebral carbon dioxide reactivity during cardiopulmonary bypass

Chapter $7 \quad$ General Discussion

Summary

Samenvatting

Dankwoord

Publications 

Chapter 1

Introduction

7 


\section{Cerebral blood flow regulation}

Maintenance of adequate cerebral blood flow is essential for normal brain function and survival. The brain receives approximately $15 \%$ of cardiac output and is responsible for approximately $20 \%$ of total body oxygen consumption [28]. Apart from oxygen delivery cerebral blood flow facilitates disposal of excess $\mathrm{CO}_{2}$ and transport of vasoactive substances. Because of the brain's limited ability to store energy cerebral blood flow needs to be controlled effectively.

To adjust cerebral blood flow to an adequate level the diameter of cerebral vessels is changed. The variable resistance to flow is encountered in the cerebral arteriolar bed mainly; the major cerebral conductance arteries are in principle noncompliant and act merely as a conduit for the pulsatile arterial flow from the aorta to the brain [18]. Cerebral blood flow (CBF) is dynamically adjusted to changes in the perfusion pressure, the metabolic activity of the brain, humoral factors and autonomic nerve activity [9].

Control of cerebral blood flow to adjust for changes in cerebral perfusion pressure is called cerebral autoregulation. In 1959, Lassen [15] first described cerebral autoregulation as the tendency of cerebral blood flow to remain approximately constant when cerebral perfusion pressure changes over a wide range, typically from 60 to $150 \mathrm{mmHg}$. In his review, Lassen showed that there is a lower blood pressure limit of cerebral blood flow autoregulation. When perfusion pressure decreases below this limit a reduction in cerebral blood flow occurs. Hypertensive forced vasodilatation had been implied in the literature for many years [24], but it was as late as the early 70's that an upper blood pressure limit of autoregulation was demonstrated [26]. Increase of perfusion pressure above this upper limit of autoregulation leads to increase of cerebral blood flow. However, unlike what would be expected, below the lower limit vasodilation is still not maximal. Häggendal et al [14] demonstrated that hypercapnia may increase cerebral blood flow even if the blood pressure is below the lower limit of autoregulation. Similarly, MacKenzie et al [16] observed, in the anesthetized cat during controlled hypotension, pial arteriolar dilation at pressures below the lower limit of autoregulation. Thus, at pressures moderately below the lower limit of autoregulation, drug-induced vasodilatation might normalize cerebral blood flow.

Disease states of the brain may impair or abolish cerebral autoregulation [22]. Autoregulation is lost in severe head injury or acute ischemic stroke, leaving surviving brain tissue unprotected against the potentially harmful effect of blood pressure changes. Likewise, autoregulation may be lost in the surroundings of a space-occupying brain lesion, be it a tumor or a hematoma. In many such disease states, autoregulation may be regained by passive hyperventilatory hypocapnia 
[25]. Autoregulation may also be impaired in neonatal brain asphyxia and infections of the central nervous system, but appears to be intact in spreading depression and migraine, despite impairment of chemical and metabolic control of cerebral blood flow. In chronic hypertension, the limits of autoregulation are shifted toward high blood pressure. Acute hypertensive encephalopathy, on the other hand, is thought to be due to autoregulatory failure at very high pressure. In long-term diabetes mellitus there may be chronic impairment of cerebral autoregulation, probably due to diabetic microangiopathy.

The exact function of cerebral autoregulation still remains unclear. Three different mechanisms are thought to play a role contributing to cerebral autoregulation [1]: metabolic, myogenic and neurogenic regulation. Metabolic regulation adjusts blood flow to both metabolic demand and oxygen supply. Myogenic regulation is the effect of transmural blood pressure changes on vascular smooth muscle tension to keep blood flow constant. Neurogenic regulation is the control of the vascular smooth muscle tension through the autonomic nervous system.

\section{Techniques used for investigating CBF regulation}

In the past, measurements performed to investigate cerebral blood flow regulation involved arterial blood pressure changes that were achieved by pharmacological means and cerebral blood flow measuring mainly depended on indicatordilution techniques, such as ${ }^{133}$ Xenon or nitrous oxide. In this situation blood pressure and cerebral blood flow data could only be recorded under steady-state conditions where data were collected over several minutes. This resulted in the well-known classical cerebral autoregulation curve (Figure 1), showing a plateau in cerebral blood flow in the case of normal autoregulation, also known as the "static" autoregulation curve [27]. If the blood flow changes significantly with either an increase or a decrease in arterial blood pressure, cerebral autoregulation is said to be impaired. If blood flow is maintained at or near the baseline level, despite a change in arterial blood pressure, cerebral autoregulation is said to be intact. 


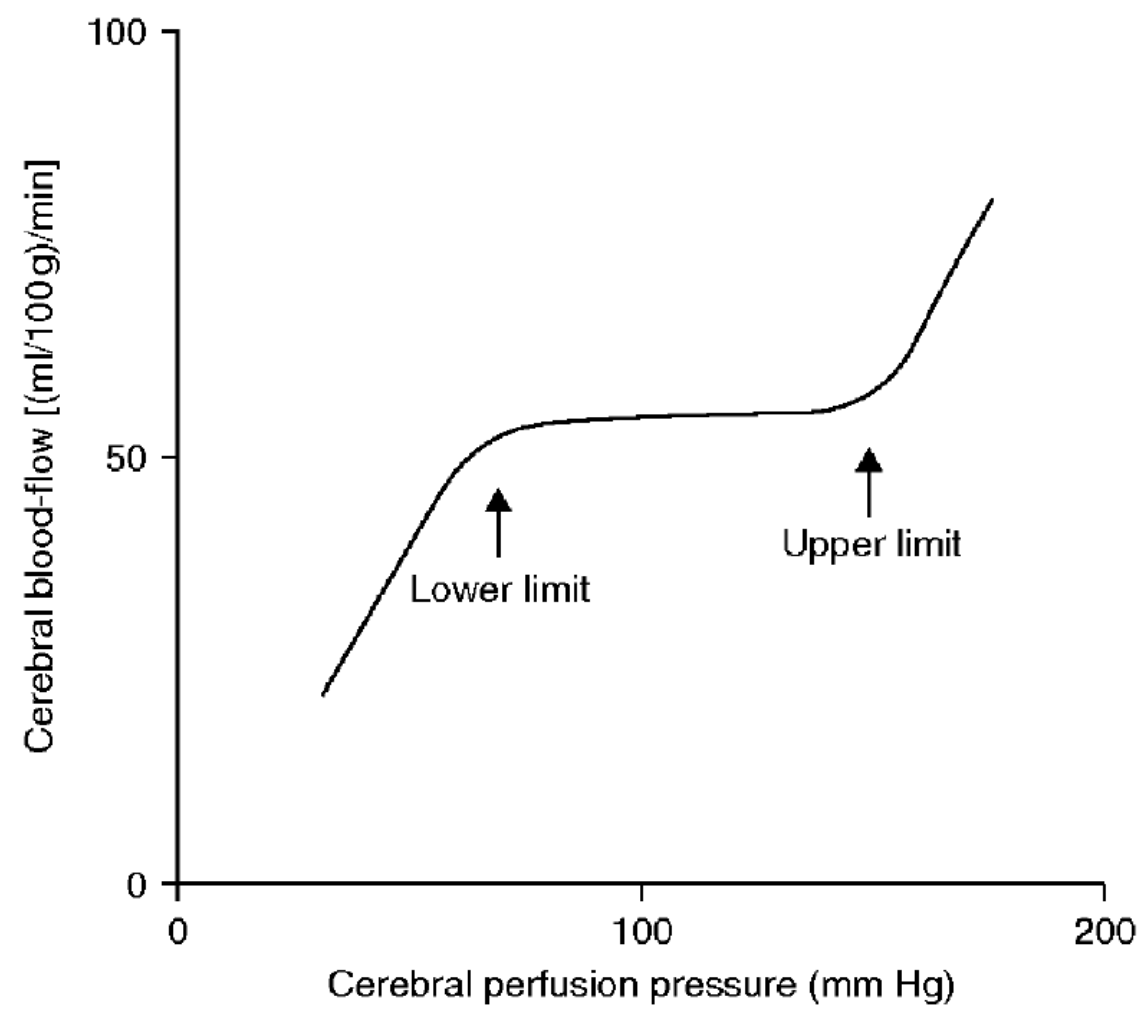

Figure 1 The relationship of cerebral blood flow versus cerebral perfusion pressure as regulated by an intact cerebral autoregulation mechanism (from Aaslid, 2006).

Only after the introduction of transcranial Doppler sonography (TCD) [5] cerebral blood flow waveform and its beat-to-beat changes could be acquired due to the high temporal resolution of TCD. However, by means of the Doppler effect only blood velocity can be measured and since the diameter of the insonated vessel is unknown cerebral blood flow can not be quantified. Under the assumption of a constant diameter in the insonated supplying vessel, cerebral blood flow velocity can be used as parameter for assessing cerebral blood flow changes. Due to the high time resolution of TCD cerebral blood flow velocity (CBFV) can now be recorded noninvasively as continuous waveform. This enables recording of transient changes in cerebral blood flow. Aaslid et al [4] were the first to study dynamic cerebral autoregulation in humans. They measured transient behavior of cerebral blood flow in response to a sudden drop in arterial blood pressure evoked by deflating leg cuffs that were inflated above systolic blood pressure for 
two minutes. To record instantaneous values of arterial blood pressure they used a servo-controlled cuff method [3].

Averaged recordings with deflating leg cuffs are shown in figure 2. Experiments have been performed at normocapnia, hypocapnia through voluntary hyperventilation and hypercapnia through breathing of $5 \% \mathrm{CO}_{2}$ in air. At each of three levels of $\mathrm{p}_{\mathrm{a}} \mathrm{CO}_{2}$ a same drop of approximately $20 \%$ in ABP has been achieved through leg cuff deflation. Approximately 10 seconds after the drop ABP has recovered to normal levels. Dynamic cerebral autoregulation has caused CBF to increase before ABP recovery. This increase is caused by adaptation of vessel resistance. According to Ohm's law cerebrovascular resistance (CVR) can be defined as ABP/CBF. During the ABP drop clearly CVR adapts to achieve fast recovery of $\mathrm{CBF}$. With hypocapnia $\mathrm{CBF}$ recovery is faster and with hypercapnia slowed compared to normocapnic conditions.
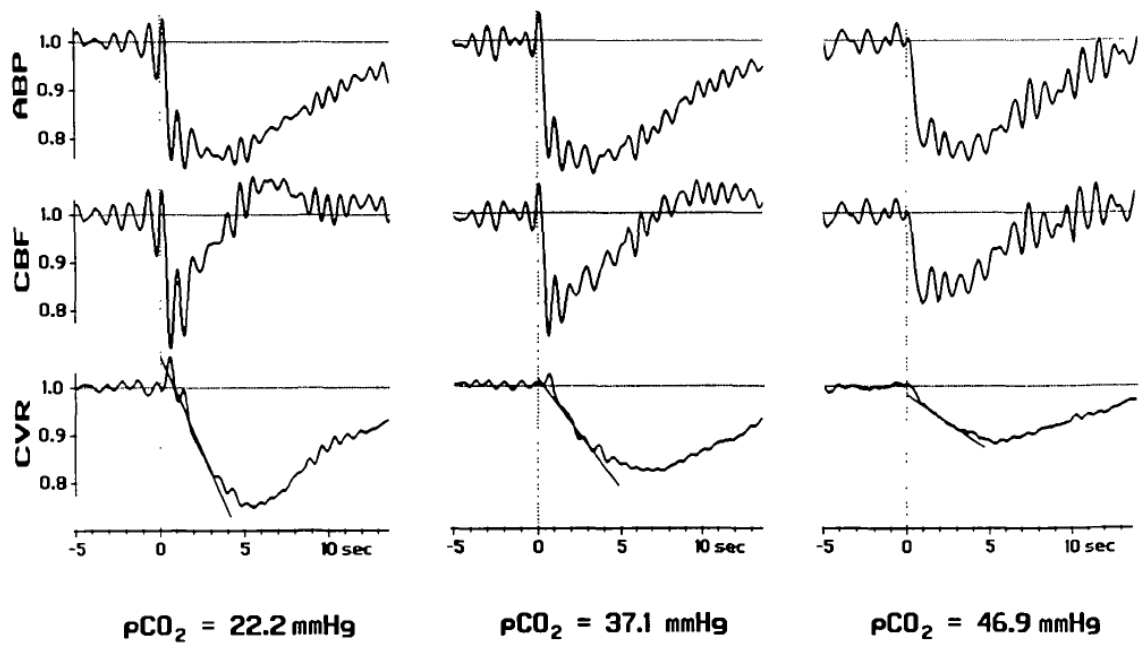

$\mathrm{PCO}_{2}=46.9 \mathrm{mmHg}$

Figure 2 Averaged recordings from leg cuff tests at three different levels of $\mathrm{p}_{\mathrm{a}} \mathrm{CO}_{2}$ (from Aaslid, 1989).

Tiecks et al [27] described a model to quantify this autoregulatory response to an ABP transient. Based on a set of model parameters ten responses to an ABP step decrease were constructed as model output. The number of each of these ten responses ranging from 0 to 9 indicate the cerebral autoregulatory index (ARI) and so quantify dynamic cerebral autoregulation (dCA). An autoregulatory index of 0 represents no cerebral autoregulation, i.e. CBFV passively follows ABP. An ARI of 9 represents fast CBFV recovery after a step change in ABP. Normal autoregulation is represented by an ARI of $5 \pm 1$. 
Different from evoking transient changes in ABP, periodic changes were induced either through controlled breathing at a frequency of $6 / \mathrm{min}$ [11] or through squat-stand maneuvers alternating 10 seconds of standing with 10 seconds of squatting [7]. These periodic changes allowed quantification of phase difference between $\mathrm{ABP}$ oscillations and oscillations in $\mathrm{CBFV}$ even in the time domain. But in general Fourier analysis is used for quantifying this phase shift. Giller [13] was the first to use Fourier analysis for the quantification of dynamic cerebral autoregulation. However, he only evaluated coherence and gain and did not address phase. Apart from evoked changes in ABP also spontaneous variation in ABP can be used to evaluate dynamic cerebral autoregulation through transfer function analysis using the Fourier transform $[8,20,29]$. This method allows in particular monitoring purposes, since no patient cooperation is needed.

\section{Fourier analysis}

Fourier analysis is a method for mathematically describing time series, e.g. an ABP recording, in terms of amplitude and frequency instead of amplitude and time. Any time series can be expressed as a sum of sine waves of specific frequencies [6]. Each sine wave of specific frequency is expressed by amplitude and phase or phase angle, expressed as part of a 360 degrees circle. A full circle of 360 degrees can also be expressed as $2 \pi$ radians. Comparing the Fourier analysis of two time series, e.g. CBFV and ABP, for each frequency the amplitude ratio is called gain and the phase difference is called phase shift. Graphs of gain and phase plotted against frequency are called gain and phase spectra. The correlation of two time series at a specific frequency can be calculated by the statistical correlation and is called coherence. A coherence of 1 means perfect correlation, whereas a coherence of zero indicates no correlation at that frequency. The graph of coherence against frequency is called coherence spectrum.

\section{Measurement means for recording cerebral autoregulation}

To assess cerebral autoregulation at least recording of blood pressure and a measure for $\mathrm{CBF}$ are required. Blood pressure can be recorded invasively via an arterial line, but also reliable noninvasive means are available nowadays. For continuous recording of CBF with possibility to use bedside calculation for monitoring purposes TCD is the best choice [28]. 


\section{Noninvasive blood pressure measurement}

In the early 1970's Peñaz [23] described a new approach to continuous noninvasive recording of blood pressure at the finger level, based on a volume-clamp method. The device was improved in its technical aspects resulting in an instrument called Finapres [17], which stands for Finger arterial pressure. Comparison with invasive recording demonstrated that finger blood pressure recording by Finapres provides an accurate estimate of the average radial blood pressure and allows a reasonably good estimate of intra-arterial blood pressure [21]. Recently, a comparative study of simultaneous Finapres recording from different fingers in the same subject showed that although differences might exist in systolic, mean and diastolic blood pressure there is excellent agreement between dynamic indices [10]. This makes noninvasive blood pressure recording of finger arterial pressure suitable for use in dynamic cerebral autoregulation evaluation.

\section{Transcranial Doppler sonography}

Transcranial Doppler sonography follows the same principles and assumptions of other applications of the Doppler effect to measure blood flow velocity in extracranial vessels: the Doppler probe generates an ultrasound beam, typically of $2 \mathrm{MHz}$ for adults which is reflected by red blood cells in a large vessel with a frequency shift that is directly proportional to the velocity of the scattering elements [2]. The frequency shift between transmitted ultrasound and reflected ultrasound allows calculation of the blood flow velocity through:

$$
v=\frac{c\left(f_{t}-f_{r}\right)}{2 f_{t} \cos \theta}
$$

where $v$ is blood flow velocity, $c$ is the speed of sound in blood, $f_{t}$ and $f_{r}$ are transmitted and received ultrasound frequency respectively and $\theta$ is the insonation angle between the ultrasound beam and the blood vessel direction at the depth of insonation.

In the presence of laminar flow, the velocity distribution across the vessel diameter will be approximately parabolic. Consequently, the reflected Doppler frequency shift will comprise a distribution of frequencies, rather than a single value. Different alternatives exist to extract meaningful velocity information. The most common approach is to apply the fast Fourier transform (FFT) algorithm to short segments (typically $\Delta \mathrm{t}=5 \mathrm{~ms}$ ) of the raw Doppler shifted signal, to obtain the spectral distribution of power at each frequency [12]. From this distribution, either the maximum frequency (i.e. maximum velocity), or its intensity-weighted mean are extracted to represent the mean velocity for the time interval $\Delta t$. The velocity distribution, and the maximum velocity envelope are normally displayed by most TCD devices as a colour coded sonogram, as represented in figure 3 . The 
maximum velocity envelope can often be recorded as analog output signal from the TCD device.

Transcranial Doppler ultrasonography depends on an acoustic window, i.e. a region of the skull with better penetration of the ultrasound beam. The transtemporal window is most relevant for studying cerebral autoregulation, since through this window the middle cerebral artery (MCA) can be insonated as well as the anterior (ACA) and posterior cerebral arteries (PCA). Mostly cerebral blood flow velocity measurements in the MCA are used for studying dCA [19].

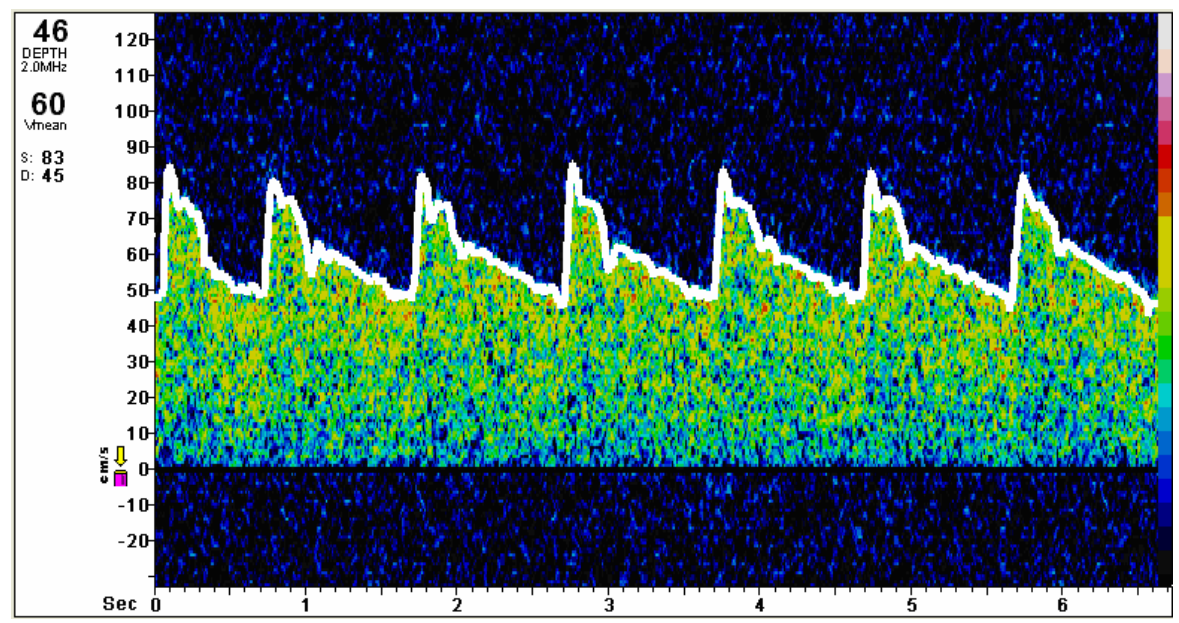

Figure 3 Sonogram showing the distribution of transcranial Doppler ultrasound shift frequencies in the middle cerebral artery. The top white envelope curve indicates the maximum velocity estimate.

\section{Aims}

Combining noninvasive finger blood pressure recording with TCD recording of cerebral blood velocity in the intracranial vessels allows studying of cerebral hemodynamics at time resolutions faster than normal heart rate. To learn more about the physiology and working mechanisms of dCA and to develop clinical applications of evaluating dCA, it is necessary to develop a reliable method to measure and quantify dCA. Being able to reliably estimate parameters of dynamic cerebral autoregulation with sufficient reproducibility by non-invasive means could improve patient diagnostics and management for several conditions. This thesis aims to improve clinical applicability of testing dynamic cerebral autoregulation. The first aim of this thesis is to evaluate the use of transfer function analy- 
sis for quantifying dynamic cerebral autoregulation. The influence of different signal analysis aspects will be studied as well as the influence of spontaneous versus evoked blood pressure variations.

Secondly our aim is to evaluate clinical application of the developed dCA analysis. Dynamic cerebral autoregulation is studied in several patient groups and the diagnostic respectively prognostic value of these test will be studied.

\section{Outline of this thesis}

Both methodological aspects and clinical applications of dynamic cerebral autoregulation are addressed in the subsequent chapters of this thesis.

Chapter 2 addresses the reproducibility of assessment of dynamic cerebral autoregulation by means of transfer function analysis. The influence of various aspects of signal analysis will be studied.

Chapter 3 evaluates the use of transfer function analysis and cerebrovascular reactivity by means of acetazolamide infusion. A patient group with small vessel disease will be studied in which dCA is evaluated before, during and after the infusion of acetazolamide. This allows to study measures of dCA in combination with cerebrovascular reactivity.

In chapter 4 the effect of a neurodegenerative disease on dCA will be studied. Results from three groups will be compared: patients with Alzheimer's disease, subjects with mild cognitive impairment and healthy elderly.

Chapter 5 describes an improved method to quantify neurovascular coupling (NVC) responses by incorporation the process of dCA in a model for NVC responses upon visual stimulation. Blood pressure related $\mathrm{CBFV}$ changes are explained by the dCA part of the model whereas visual stimulus related CBFV changes are explained by the NVC part.

In chapter 6 a method is described for evaluating dCA during cardiopulmonary bypass where blood pressure variability is not influenced through control of heart rate. It is tested whether externally evoked variability of blood pressure through changing pump flow is adequate in creating sufficient CBFV variability to enable the study of dCA. 
In chapter 7 in a general discussion the methods and clinical applications described in this thesis will be discussed.

\section{References}

1. Aaslid R. Cerebral autoregulation and vasomotor reactivity. Front Neurol Neurosci 21: 216-228, 2006.

2. Aaslid R. Transcranial Doppler sonography. New York: Springer, 1986.

3. Aaslid R, and Brubakk AO. Accuracy of an ultrasound Doppler servo method for noninvasive determination of instantaneous and mean arterial blood pressure. Circulation 64: 753-759, 1981.

4. Aaslid R, Lindegaard KF, Sorteberg W, and Nornes H. Cerebral autoregulation dynamics in humans. Stroke 20: 45-52, 1989.

5. Aaslid R, Markwalder TM, and Nornes H. Noninvasive transcranial Doppler ultrasound recording of flow velocity in basal cerebral arteries. J Neurosurg 57: 769-774, 1982.

6. Bendat JS, and Piersol AG. Random data: Analysis and Measurement Procedures. New York: Wiley, 1971.

7. Birch AA, Dirnhuber MJ, Hartley-Davies R, Iannotti F, and Neil-Dwyer G. Assessment of autoregulation by means of periodic changes in blood pressure. Stroke 26: 834-837, 1995.

8. Blaber AP, Bondar RL, Stein F, Dunphy PT, Moradshahi P, Kassam MS, and Freeman R. Transfer function analysis of cerebral autoregulation dynamics in autonomic failure patients. Stroke 28: 1686-1692, 1997.

9. Busija DW, and Heistad DD. Factors involved in the physiological regulation of the cerebral circulation. Rev Physiol Biochem Pharmacol 101: 161-211, 1984.

10. Chin KY, and Panerai RB. Comparative study of Finapres devices. Blood Pressure Monitoring 17: 171-178, 2012.

11. Diehl RR, Linden D, Lucke D, and Berlit P. Phase relationship between cerebral blood flow velocity and blood pressure. A clinical test of autoregulation. Stroke 26: 1801-1804, 1995.

12. Evans DH, and McDicken WN. Doppler Ultrasound: Physics, Instrumentation and Signal Processing. Chichester: John Wiley, 2000, p. 427.

13. Giller CA. The frequency-dependent behavior of cerebral autoregulation. Neurosurgery 27: 362-368, 1990.

14. Haggendal E, and Johansson B. Effects of arterial carbon dioxide tension and oxygen saturation on cerebral blood flow autoregulation in dogs. Acta Physiol Scand Suppl 258: 27-53, 1965. 
15. Lassen NA. Cerebral Blood Flow and Oxygen Consumption in Man. Physiol Rev 39: 183-238, 1959.

16. MacKenzie ET, Farrar JK, Fitch W, Graham DI, Gregory PC, and Harper AM. Effects of hemorrhagic hypotension on the cerebral circulation. I. Cerebral blood flow and pial arteriolar caliber. Stroke 10: 711-718, 1979.

17. Molhoek GP, Wesseling KH, Settels JJ, van Vollenhoven E, Weeda HW, de Wit B, and Arntzenius AC. Evaluation of the Penaz servo-plethysmomanometer for the continuous, non-invasive measurement of finger blood pressure. Basic Res Cardiol 79: 598-609, 1984.

18. Olufsen MS, Nadim A, and Lipsitz LA. Dynamics of cerebral blood flow regulation explained using a lumped parameter model. Am J Physiol Regul Integr Comp Physiol 282: R611-622, 2002.

19. Panerai RB. Transcranial Doppler for evaluation of cerebral autoregulation. Clin Auton Res 19: 197-211, 2009.

20. Panerai RB, Dawson SL, and Potter JF. Linear and nonlinear analysis of human dynamic cerebral autoregulation. Am J Physiol 277: H1089-1099, 1999.

21. Parati G, Casadei R, Groppelli A, Di Rienzo M, and Mancia G. Comparison of finger and intra-arterial blood pressure monitoring at rest and during laboratory testing. Hypertension 13: 647-655, 1989.

22. Paulson OB, Strandgaard S, and Edvinsson L. Cerebral autoregulation. Cerebrovasc Brain Metab Rev 2: 161-192, 1990.

23. PeñazJ. Photoelectric measurement of blood pressure, volume and flow in the finger. In: Digest of the International Conference of Medicine and Biological Engineering. Dresden: Conference Committee of the Xth International Conference on Medicine and Biological Engineering, 1973, p. 104.

24. Pickering GW. The pathogenesis of malignant hypertension. Circulation 6: 599-612, 1952.

25. Raichle ME, and Plum F. Hyperventilation and Cerebral Blood Flow. Stroke 3: 566-575, 1972.

26. Symon L, Held K, and Dorsch NW. A study of regional autoregulation in the cerebral circulation to increased perfusion pressure in normocapnia and hypercapnia. Stroke 4: 139-147, 1973.

27. Tiecks FP, Lam AM, Aaslid R, and Newell DW. Comparison of static and dynamic cerebral autoregulation measurements. Stroke 26: 10141019, 1995.

28. Willie CK, Colino FL, Bailey DM, Tzeng YC, Binsted G, Jones LW, Haykowsky MJ, Bellapart J, Ogoh S, Smith KJ, Smirl JD, Day TA, Lucas SJ, Eller LK, and Ainslie PN. Utility of transcranial Doppler ultrasound 
Chapter 1

for the integrative assessment of cerebrovascular function. Journal of Neuroscience Methods 196: 221-237, 2011.

29. Zhang R, Zuckerman JH, Giller CA, and Levine BD. Transfer function analysis of dynamic cerebral autoregulation in humans. Am J Physiol274: H233-241, 1998. 
Chapter 2

Dynamic cerebral autoregulation: different signal processing methods without influence on results and reproducibility

E D Gommer, E Shijaku, W H Mess and J P H Reulen. Dynamic cerebral autoregulation: different signal processing methods without influence on results and reproducibility.

Med Biol Eng Comput 2010 Dec; 48(12):1243-50. 


\section{Abstract}

Cerebral autoregulation controls cerebral blood flow under changing cerebral perfusion pressure. Standards for measurement and analysis of dynamic cerebral autoregulation (dCA) are lacking. In this study dCA reproducibility, quantified by intraclass correlation coefficient, is evaluated for different methodological approaches of transfer function analysis (TFA) and compared with multimodal pressure flow analysis (MMPF).

$\mathrm{dCA}$ parameters were determined in 19 healthy volunteers during three $15 \mathrm{~min}-$ utes lasting epochs of spontaneous breathing. Every spontaneous breathing epoch was followed by 5 minutes of paced breathing at 6 cycles $/ \mathrm{min}$. These 6 measurements were performed in both a morning and an afternoon session. Analysis compared raw data pre-processing by mean subtraction versus smoothness priors detrending. Furthermore, spectral density estimation by averaging of subsequent time windows versus smoothing the whole recording spectrum.

No significant influence of pre-processing and spectral estimation on dCA parameters was found. Therefore, there seems to be no need to prescribe a specific signal-processing regime. Poor reproducibility of gain and phase was found for TFA as well as for MMPF. Based on reproducibility, no preference can be made for morning versus afternoon measurements. Neither for spontaneous versus paced breathing. Finally, reproducibility results are not in favour of TFA or MMPF. 


\section{Introduction}

Under normal conditions, despite variations in cerebral perfusion pressure (CPP), cerebral blood flow is kept relatively constant in correspondence with the metabolic needs of the brain. The control mechanism that compensates for CPP variations is called cerebral autoregulation $[2,19,24]$. Various methods have been described to characterize this control system. A sudden step-wise decrease in arterial blood pressure evokes a response, characterized by a few seconds lasting decreased flow followed by a relatively slow return to the base level. This CBFVresponse can be interpreted as a kind of step-response of the control system and can be quantified by the so-called autoregulatory index (ARI) [30] ranging between 0 and 9 . Another approach to quantify the dynamics of cerebral autoregulation $(\mathrm{dCA})[4,22,33]$ is transfer function analysis (TFA) of the presumed linear control system with arterial blood pressure (ABP) as the input and cerebral blood flow velocity (CBFV) as the output signal. The transfer function gain and phase are estimated from the auto- and cross spectra of $\mathrm{ABP}$ and CBFV and the strength of the linear relation between $\mathrm{ABP}$ and $\mathrm{CBFV}$ is expressed in the system's coherence. From the step response function resulting from this transfer function the system's ARI can be determined using least squares estimation. For each of the ten ARI responses the sum of squared differences with the TFA response is determined. A parabolic interpolation through these ten values can be performed to estimate the value of ARI to one decimal space [23] at the minimum of the parabola.

A recently published and different approach to overcome some difficulties with Fourier-based spectral analysis is the multimodal pressure flow (MMPF) analysis $[15,20]$. The authors showed that MMPF analysis may be less sensitive to problems with data non-stationarities and trends [15]. In a study on traumatic brain injury patients better reproducibility was shown with MMPF phase compared to ARI [11]. Also non-linear approaches have been tried to evaluate dCA [13, 17], but clear benefits have not been shown yet.

A limiting factor in (clinical) use of quantitative $\mathrm{dCA}$ is the high variability observed in calculated parameters, such as gain, phase and ARI $[6,16,23,27]$. This high variability is likely to limit reproducibility of these measurements. Brodie et al [6] showed poor ARI reproducibility with an intraclass correlation (ICC) below 0.5 . It is unknown if reproducibility may be influenced by procedural and methodological options.

Currently, there is neither a procedural nor a methodological standard to compute dCA. A procedural aspect is the choice between using spontaneous [22, 33] and induced blood pressure variations e.g. by paced breathing at a frequency of 
$6 / \min [10,26]$. Paced breathing requires cooperation of the patient whereas spontaneous blood pressure variations can be recorded without specific instructions. The same holds for squat-stand manoeuvres, although this seems a promising addition [7]. Another procedural aspect concerns possible circadian variations in dCA. Ainslie et al reported a significant lowering in ARI from evening to morning [3]. Therefore, it might be necessary to standardise the time of day for dCA recording.

For TFA different signal processing techniques can be applied and the most common will be evaluated in this study. First, before applying Fourier transform, offset and/or slow trends are removed either by subtracting the mean or by sophisticated detrending procedures to remove also very low frequencies. Second, for spectral estimation two approaches can be used: either the spectral averaging technique, also known as the Welch technique [32], in which spectra of subsequent epochs are averaged or spectral smoothing in which the spectra are smoothed with triangular smoothing windows [31].

In this study the effect on reproducibility will be evaluated for the different TFA analysis options and for the MMPF method. A major question is whether results of MMPF compared to TFA analysis are more consistent and show less variability. To reveal effects of the measurement conditions also spontaneous vs. paced breathing and morning vs. afternoon recordings will be compared.

\section{Methods}

\section{Subjects and measurements}

Nineteen healthy subjects (14M/5F) between 18 and 53 years old (mean 28) voluntarily participated in the study. All were free of known cardiovascular, pulmonary and cerebrovascular disorders. Each subject was measured in supine position on two different days, one day in the morning at $10 \mathrm{am}$ and a different day one week apart in the afternoon at $2 \mathrm{pm}$. The order of day was randomly assigned. Each recording session lasted about one hour. A session consisted of three successive runs each consisting of 15 minutes of spontaneous breathing followed by 5 minutes of $6 / \mathrm{min}$ frequency paced breathing.

A one channel electrocardiogram (ECG) was measured by an in-house made portable ECG-amplifier (IDEE Maastricht ${ }^{\circledR}$, the Netherlands). Continuous arterial blood pressure (ABP) was measured using a non-invasive finger blood pressure monitor (Portapres ${ }^{\circledR}$, TNO, Amsterdam, the Netherlands) commonly used in studies on dynamic cerebral autoregulation. The appropriate finger cuff (size: small, medium or large) was placed on the left hand middle finger. During 
the recordings the Physiocal procedure, an intermittently occurring calibration routine, was switched off. To measure cerebral blood flow velocity (CBFV) simultaneously in the right and left middle cerebral artery a Transcranial Dopplersonography device (DWL Multi-Dop ${ }^{\circledR}$ X4, Sipplingen, Germany) was used. The two $2 \mathrm{MHz}$-probes were held in position by a special head frame.

\section{Data analysis}

ECG, ABP and right and left CBFV signals were recorded on a DAT-tape(TEACrecorder) and thereafter offline digitised and stored on a PC with an in-house made acquisition and analysis software package (AFOnew version 2.33, IDEE Maastricht ${ }^{\circledR}$, the Netherlands). The sampling frequency for each signal was set to $250 \mathrm{~Hz}$. An automatic algorithm detected the ECG R-waves and determined the subsequent diastolic, mean and systolic blood pressure and corresponding enddiastolic, mean and peak-systolic CBFV. Artefacts were removed manually by linear interpolation. The beat-to-beat values of $\mathrm{ABP}$ and $\mathrm{CBFV}$ were resampled with $5 \mathrm{~Hz}$ using spline-interpolation [22]. From the ABP the overall mean value was subtracted and the CBFVs were normalised with respect to the mean. This resulted in zero-mean signals suited for spectral analysis to estimate the transfer function. The transfer function was calculated by

$$
H(f)=\frac{S_{x y}(f)}{S_{x x}(f)}
$$

where $S_{x x}(f)$ is the autospectrum of changes in arterial blood pressure and $S_{x y}(f)$ is the cross-spectrum between the ABP- and CBFV-signals. The transfer function magnitude $|H(f)|$ and phase spectrum $\Phi(f)$ were derived from the real part $H_{R}(f)$ and imaginary part $H_{I}(f)$ of the complex transfer function as

$$
\begin{aligned}
& |H(f)|=\sqrt{\left[H_{R}(f)\right]^{2}+\left[H_{I}(f)\right]^{2}} \\
& \Phi(f)=\arctan \frac{H_{I}(f)}{H_{R}(f)}
\end{aligned}
$$

The squared coherence function $\gamma^{2}(\mathrm{f})$ was estimated by

$$
\gamma^{2}(f)=\frac{\left|S_{x y}(f)\right|^{2}}{S_{x x}(f) S_{y y}(f)}
$$

where $S_{y y}(f)$ is the autospectrum of changes in cerebral blood flow velocity. The squared coherence reflects the strength of the linear relationship between $\mathrm{ABP}$ and CBFV for each frequency on a scale from 0 to 1 . 
Table 1. Summary of parameter choices in transfer function estimation.

\begin{tabular}{cccccc}
\hline $\begin{array}{c}\text { Combina- } \\
\text { tion }\end{array}$ & $\begin{array}{c}\text { Detrend- } \\
\text { ing }\end{array}$ & $\begin{array}{c}\text { Window } \\
\text { length } \\
{[\text { samples }]}\end{array}$ & $\begin{array}{c}\text { Window } \\
\text { duration } \\
{[\mathrm{s}]}\end{array}$ & $\begin{array}{c}\text { Triangular } \\
\text { smoothing } \\
\text { window } \\
\text { width }\end{array}$ & $\begin{array}{c}\text { Smoothing } \\
\text { bandwidth } \\
{[\mathrm{Hz}]}\end{array}$ \\
\hline I & $\begin{array}{c}\text { Mean } \\
\text { subtrac- } \\
\text { tion } \\
\text { Smooth- }\end{array}$ & 256 & 51.2 & 3 & 0.04 \\
II & 256 & 51.2 & 3 & 0.04 \\
III & $\begin{array}{c}\text { ness priors } \\
\text { Mean } \\
\text { subtrac- } \\
\text { tion } \\
\text { Smooth- }\end{array}$ & 2048 & 409.6 & 31 & 0.04 \\
IV & 2048 & 409.6 & 31 & 0.04 \\
\hline
\end{tabular}

To reduce variance of the spectral estimates of the transfer function either spectral averaging or spectral smoothing can be performed. Spectral averaging was first introduced by Welch [32] and averaged spectra were calculated over multiple half overlapping epochs. An epoch length of 50 seconds is chosen to result in an adequate frequency resolution of at most $0.02 \mathrm{~Hz}$. Spectral smoothing reduces variance by filtering the spectrum with a triangular window averaging over a frequency band. In this case the spectrum is calculated over almost the entire measurement resulting in a much finer frequency resolution. In this study four different combinations of parameter settings (table 1) for detrending were tested. In method I and III only mean subtraction was performed, whereas in method II and IV smoothness priors detrending [29] with a $0.04 \mathrm{~Hz}$ cut-off frequency $(\lambda=500)$ was used to remove very low frequency trends. Each data epoch was windowed with a Hanning-window. In method I and II half overlapping $51.2 \mathrm{~s}$ long epochs of 256 data points with a $5 \mathrm{~Hz}$ resampling rate were used; in method III and IV 2048 points, corresponding to a 409.6 s epoch length. In method I and II the spectrum was smoothed with a 3-point triangular moving average window corresponding to a full width half max bandwidth of $0.04 \mathrm{~Hz}$; in III and IV a 31 points wide triangular window, also corresponding to a bandwidth of $0.04 \mathrm{~Hz}$ $[18,31]$.

From the resulting transfer functions, mean coherence, phase and gain were calculated in the $0.04-0.16 \mathrm{~Hz}$ frequency range. The $0.04 \mathrm{~Hz}$ lower border has been chosen because the limited duration of the recordings makes interpretation of results below this frequency difficult. The upper frequency is chosen to in- 
clude the major frequency range of dCA. The choice of the $0.04-0.16 \mathrm{~Hz}$ frequency interval does not introduce new problems of standardization sincein this paper all methodologies and protocols are mutually compared using this fixed interval. Furthermore, the graphical presentation of our results as a function of frequency allows estimation for other frequency bands.

Paced breathing analysis was only possible for combinations I and II (table 1) because of the limited 5 min duration of these recordings.

ARI was calculated from the first 7 seconds of the step response function using a previously described method [16]. The step response to calculate the ARI is obtained by integrating the impulse response, being the inverse Fourier transform of the estimated transfer function. From the ABP and CBFV waveforms the critical closing pressure and RAP were determined per beat using linear regression [21]. Before regression, individual $\mathrm{ABP}$ and $\mathrm{CBFV}$ beat waveforms were aligned for maximum correlation. RAP $(\mathrm{mmHgs} / \mathrm{cm})$ is the reciprocal slope of the regression line.

\section{Coherence criterion}

To allow reliable interpretation of dCA results coherence levels needs to exceed a minimum level. This minimum level, above which coherence differs significantly from zero, depends on the degrees of freedom of the spectral estimation procedure (computation in appendix A). Computation based on the degrees of freedom resulted in a minimum needed squared coherence level of 0.06 for the 15 -minute spontaneous breathing episodes and of 0.2 for the 5 -minute $6 / \mathrm{min}$ breathing periods.

\section{Multimodal pressure flow analysis}

For multimodal pressure flow analysis a recently improved method was used including ensemble empirical mode decomposition (EEMD) $[14,20]$. To extract spontaneous oscillations in $\mathrm{ABP}$ and $\mathrm{CBFV}$ empirical mode decomposition was used to decompose $\mathrm{ABP}$ and $\mathrm{CBFV}$ signals into intrinsic modes. For the EEMD this decomposition was repeated 100 times with added white noise having an amplitude of $10 \%$ of the standard deviation of the signal. The resulting 100 realizations of intrinsic mode functions were averaged per mode. Only component 3 and 4 were used for the analysis, since these were the only components with frequencies in the range from $0.04-0.16 \mathrm{~Hz}$. To compare the values for phase and gain with the results from the transfer function analysis averages were taken over the whole measurement course. A recently modified transfer function analysis (MTFA) also was applied [15]. This method evaluates gain and phase with transfer 
function analysis when instead of the beat-to-beat ABP and CBFV signals, the intrinsic mode functions of the MMPF were used.

\section{Statistical analysis}

The non-parametric Wilcoxon-signed rank test was used to test for left/right and morning/afternoon differences for each method. Reproducibility was quantified by the intraclass correlation coefficient (ICC) using the generalisability theory [8, 28]. ICC, defined as the sum of variances except for repeated measurements components divided by the total variance, was calculated [25] using the GENOVA (version 2.2,1984) program. Although ICC is specific to the population studied, it does give clear indications of inter-method reproducibility being a major purpose of this study.

\section{Results}

\section{Spontaneous blood pressure variations}

From all recorded data ( 19 subjects ${ }^{*} 2$ sessions $* 1$ hour $=39$ hours) only a single period of 20 minutes had to be excluded because of bad signal quality. Pair wise comparison of coherence, gain and phase parameters between left and right CBFV recordings did not reveal significant differences. Therefore, per individual and recording session left and right side data were pooled for further analysis. Morning and afternoon dCA parameters for the four analysis methods are given in the supplemental material (table S1). MMPF results are presented for intrinsic mode functions 3 and 4. The mean frequencies of ABP and CBFV for these components 3 and 4 and their standard deviations are indicative for narrowbanded signals. Also the frequencies of intrinsic mode functions 3 and 4 match very well for $A B P$ and $C B F V$. 
Reproducibility of dynamic cerebral autoregulation

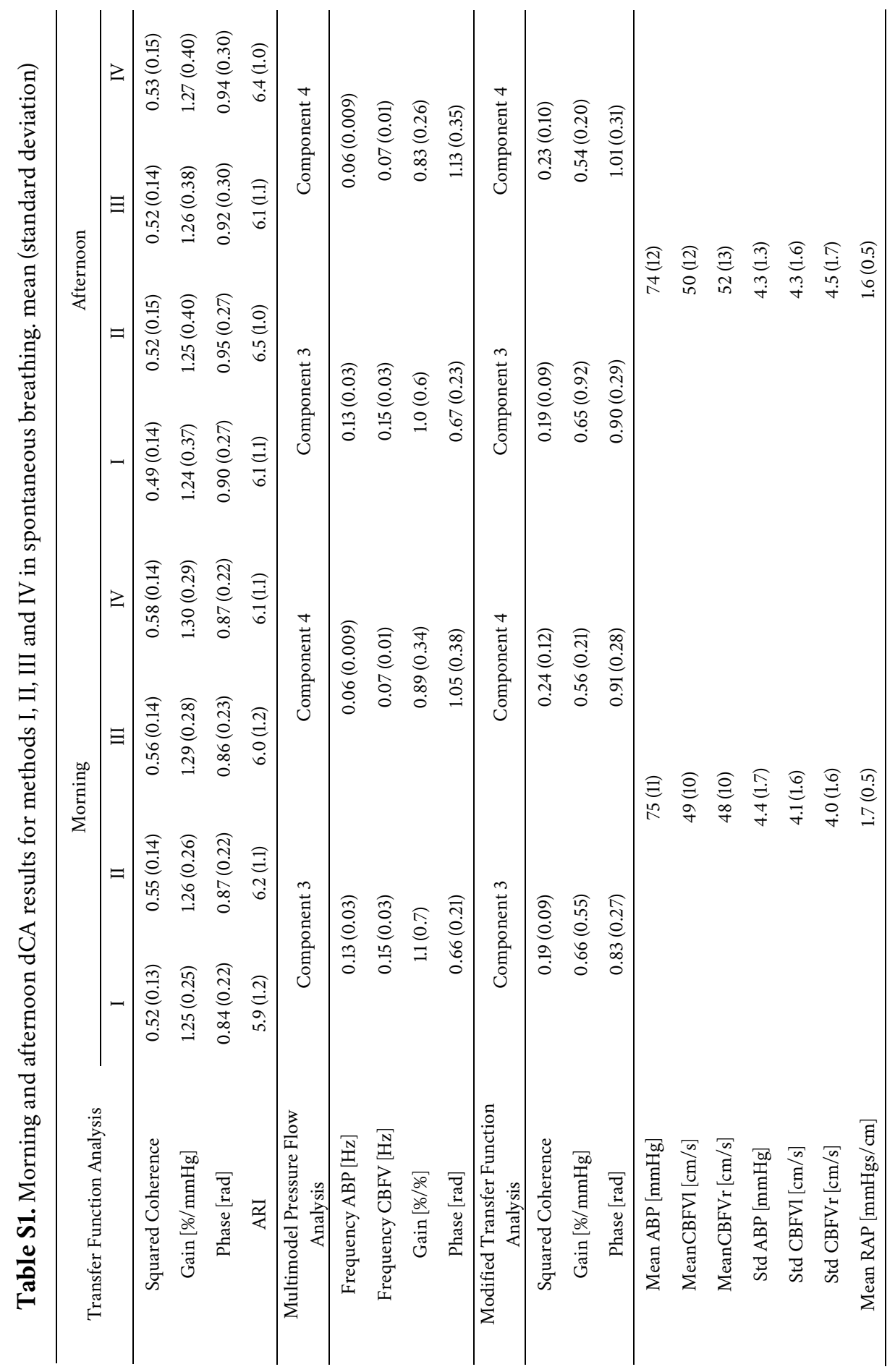




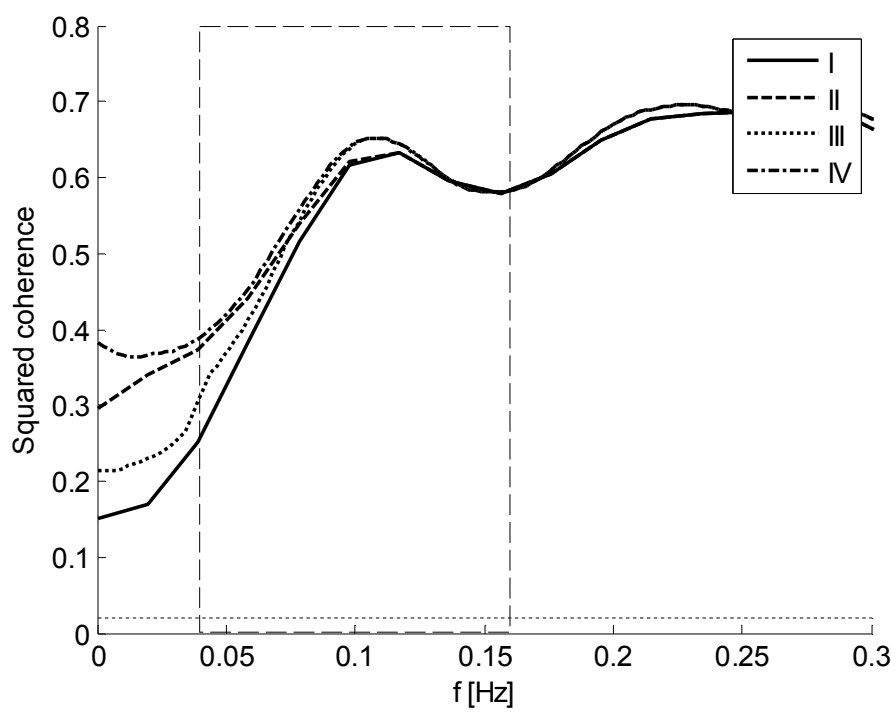

Figure 1. Group-averaged spectral squared coherence plot for method I (solid), II (dashed), III (dotted) and IV (dash-dotted) during spontaneous breathing. Marked by the dashed box is the frequency range analysed. Marked by the dotted line is the coherence threshold value.

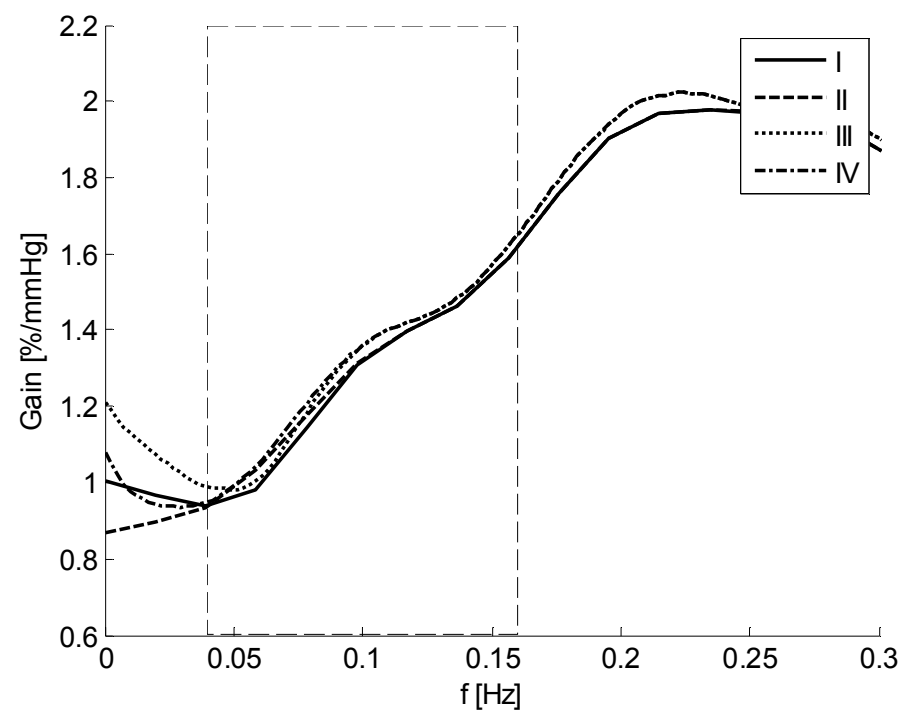

Figure 2. Group-averaged spectral gain plot for method I (solid), II (dashed), III (dotted) and IV (dash-dotted) during spontaneous breathing. Marked by the dashed box is the frequency range analysed. 
The listed values for std $\mathrm{ABP}$ and std CBFV represent the standard deviation over time of respectively the mean $\mathrm{ABP}$ and mean $\mathrm{CBFV}$. For none of the methods significant differences were found between morning and afternoon values.

In figures 1, 2 and 3 the group averages of coherence, gain and phase spectra are presented for spontaneous breathing episodes for four different analysis schemes. There is good correspondence between the curves of the four analysis methods and also the MMPF values for phase are close to these curves at the corresponding frequencies for component 3 and 4 . The MTFA values are also in good correspondence when taking into account that these are averages the 0.04$0.16 \mathrm{~Hz}$ frequency range.

\section{Paced 6/min breathing}

Paced breathing lasted 5 minutes. For this reason the 2048-point spectral smoothing method was not applicable. In table $\mathrm{S} 2$ of the supplemental material $\mathrm{dCA}$ parameters are presented for morning and afternoon for the two analysis methods. No significant differences were found between morning and afternoon. The group averages of the phase spectra are plotted in figure 4 for the paced breathing episodes for the two detrending methods. For the $0.1 \mathrm{~Hz}$ frequency component there is good correspondence between the transfer function analysis methods and the MMPF and MTFA method.

\section{Comparison of signal processing methods}

In total 48 independent sample comparisons were made for the four different signal-processing methods in spontaneous breathing. Only for coherence statistically significant differences could be shown. Coherence was significantly lower for method I compared to III ( $\mathrm{p}=0.04)$ and IV ( $\mathrm{p}=0.005)$.

\section{Spontaneous versus paced breathing}

In total 8 pairwise comparisons of dCA parameters for spontaneous versus paced breathing were performed. They showed that phase and ARI were significantly higher in paced versus spontaneous breathing using method II both for the morning $(\mathrm{p}=0.001)$ and afternoon session $(\mathrm{p}=0.01)$ and only in the morning $(\mathrm{p}=0.006)$ for method I. 


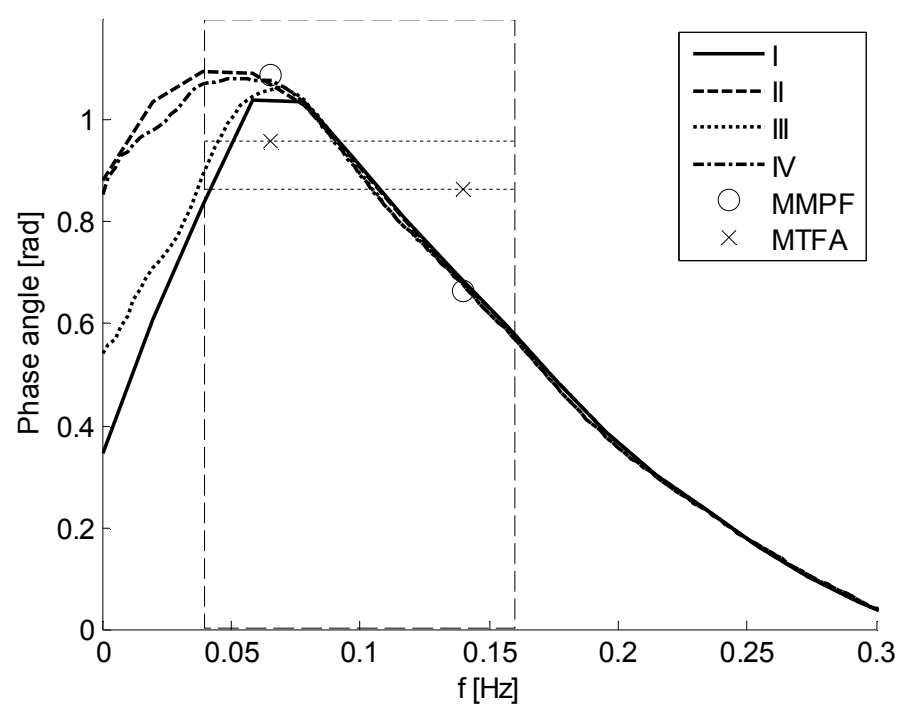

Figure 3. Group-averaged spectral phase plot for TFA method I (solid), II (dashed), III (dotted) and IV (dash-dotted), MMPF method and MTFA method during spontaneous breathing. Marked by the dashed box is the frequency range analysed.

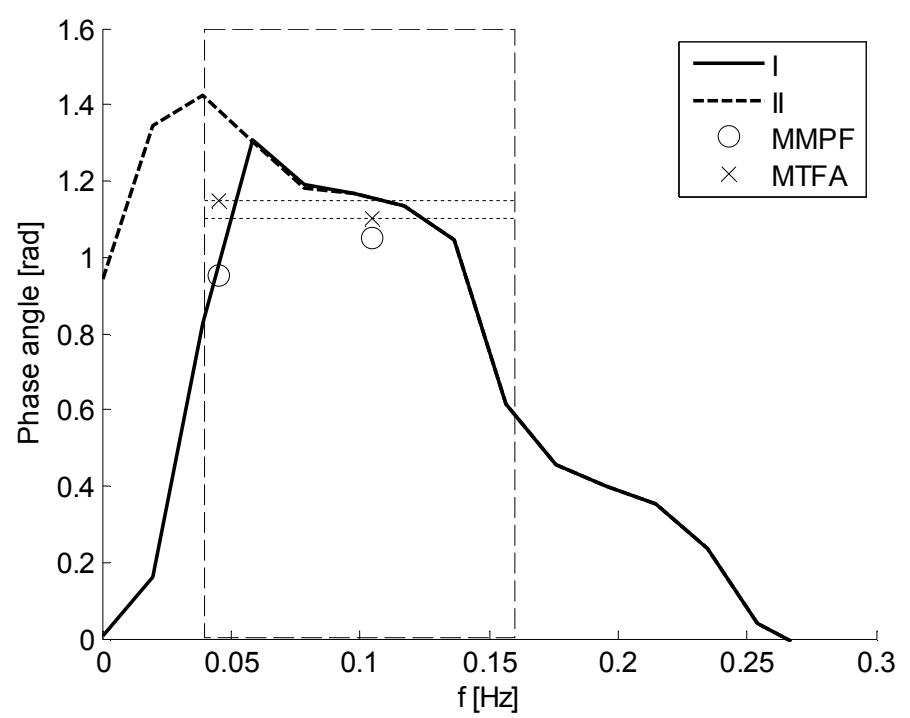

Figure 4. Group-averaged spectral phase plot for TFA with (solid) and without (dashed) using smoothness priors detrending and MMPF and MTFA method during $6 /$ min paced breathing. Marked by the dashed box is the frequency range analysed. 


\section{Reproducibility of dynamic cerebral autoregulation}

Table S2. Morning and afternoon dCA results for methods I and II in paced breathing. mean (standard deviation)

\begin{tabular}{|c|c|c|c|c|}
\hline \multirow[b]{2}{*}{ Transfer Function Analysis } & \multicolumn{2}{|c|}{ Morning } & \multicolumn{2}{|c|}{ Afternoon } \\
\hline & I & II & I & II \\
\hline Squared Coherence & $0.53(0.13)$ & $0.57(0.14)$ & $0.52(0.14)$ & $0.57(0.14)$ \\
\hline Gain $[\% / \mathrm{mmHg}]$ & $1.29(0.36)$ & $1.34(0.37)$ & $1.34(0.41)$ & $1.37(0.40)$ \\
\hline Phase [rad] & $1.05(0.47)$ & $1.09(0.44)$ & $1.11(0.49)$ & $1.15(0.48)$ \\
\hline ARI & $6.6(1.0)$ & $6.8(0.95)$ & $6.5(1.3)$ & $6.8(1.1)$ \\
\hline $\begin{array}{l}\text { Multimodel Pressure Flow } \\
\text { Analysis }\end{array}$ & Component 3 & Component 4 & Component 3 & Component 4 \\
\hline Frequency $\mathrm{ABP}[\mathrm{Hz}]$ & $0.10(0.01)$ & $0.04(0.01)$ & $0.10(0.009)$ & $0.04(0.01)$ \\
\hline Frequency CBFV [Hz] & $0.11(0.01)$ & $0.05(0.01)$ & $0.11(0.01)$ & $0.05(0.01)$ \\
\hline Gain [\%/\%] & $0.96(0.37)$ & $0.89(0.26)$ & $0.87(0.34)$ & $0.87(0.31)$ \\
\hline Phase [rad] & $1.0(0.6)$ & $0.7(1.4)$ & $1.1(0.5)$ & $1.2(1.0)$ \\
\hline $\begin{array}{l}\text { Modified Transfer Function } \\
\text { Analysis }\end{array}$ & Component 3 & Component 4 & Component 3 & Component 4 \\
\hline Squared Coherence & $0.28(0.08)$ & $0.23(0.11)$ & $0.30(0.07)$ & $0.24(0.12)$ \\
\hline Gain $[\% / \mathrm{mmHg}]$ & $0.68(0.23)$ & $0.51(0.41)$ & $0.66(0.33)$ & $0.54(0.35)$ \\
\hline Phase [rad] & $1.1(0.5)$ & $1.1(0.8)$ & $1.1(0.5)$ & $1.2(0.8)$ \\
\hline Mean ABP $[\mathrm{mmHg}]$ & & & & \\
\hline Mean CBFV L $[\mathrm{cm} / \mathrm{s}]$ & & & & \\
\hline Mean CBFV R $[\mathrm{cm} / \mathrm{s}]$ & & & & \\
\hline Std ABP $[\mathrm{mmHg}]$ & & & & \\
\hline Std CBFV L $[\mathrm{cm} / \mathrm{s}]$ & & & & \\
\hline Std CBFV R $[\mathrm{cm} / \mathrm{s}]$ & & & & \\
\hline Mean RAP [mmHgs/cm] & & & & \\
\hline
\end{tabular}




\section{Reproducibility analysis}

As can be seen in the tables S3 and S4 (supplemental material), all ICC's have wide overlapping confidence intervals. The confidence interval width can only be decreased by inclusion of many more $(>100)$ subjects. Only then significance of the apparent differences between ICC values may be deduced. Now, only the parameters with ICC values above 0.9 can be considered reproducible at an acceptable level. During spontaneous breathing, this only holds for CBFV in the morning and afternoon (0.93), BP in the morning (0.91) and RAP (0.91) in the afternoon. In paced breathing this only holds for CBFV (0.94) and RAP (0.92) in the afternoon. None of the autoregulation parameters shows adequate reproducibility.

\section{Discussion}

Our evaluation of four different signal-processing strategies shows that there is only limited influence on dCA parameters. Spectral smoothing [18] compared to epoch averaging [32] was suggested to improve detection of very low frequency cerebral haemodynamic oscillations. Our results only show slight improvement of coherence using spectral smoothing without an effect on gain and phase. Also the smoothness priors detrending method [29] results in higher coherence in the very low frequency range with hardly any effect on gain and phase. It seems therefore that the gain and phase parameters in the frequency range from 0.04$0.16 \mathrm{~Hz}$ are very robust for the different signal processing methods applied.

Impaired early morning (6-8am) cerebral autoregulation was shown compared to evening (6-8pm) measurements in 20 healthy subjects [3]. To assess ARI the investigators used the thigh cuff inflation-deflation method. We investigated our 19 subjects in the morning (10 am) and afternoon ( $2 \mathrm{pm})$ and could not demonstrate significant differences in dCA parameters using transfer function analysis. The ARI values extracted from the transfer function neither showed morning versus afternoon differences. Based on our results, no preference can be made for morning or afternoon dCA measurements. However, we can not rule out the possible influence on dCA due to circadian rhythms. Neither can we counter the morning-evening differences shown by Ainslie et al, since our morning and afternoon measurements were at different times (10 am and $2 \mathrm{pm}$ ). 
Reproducibility of dynamic cerebral autoregulation




Chapter 2

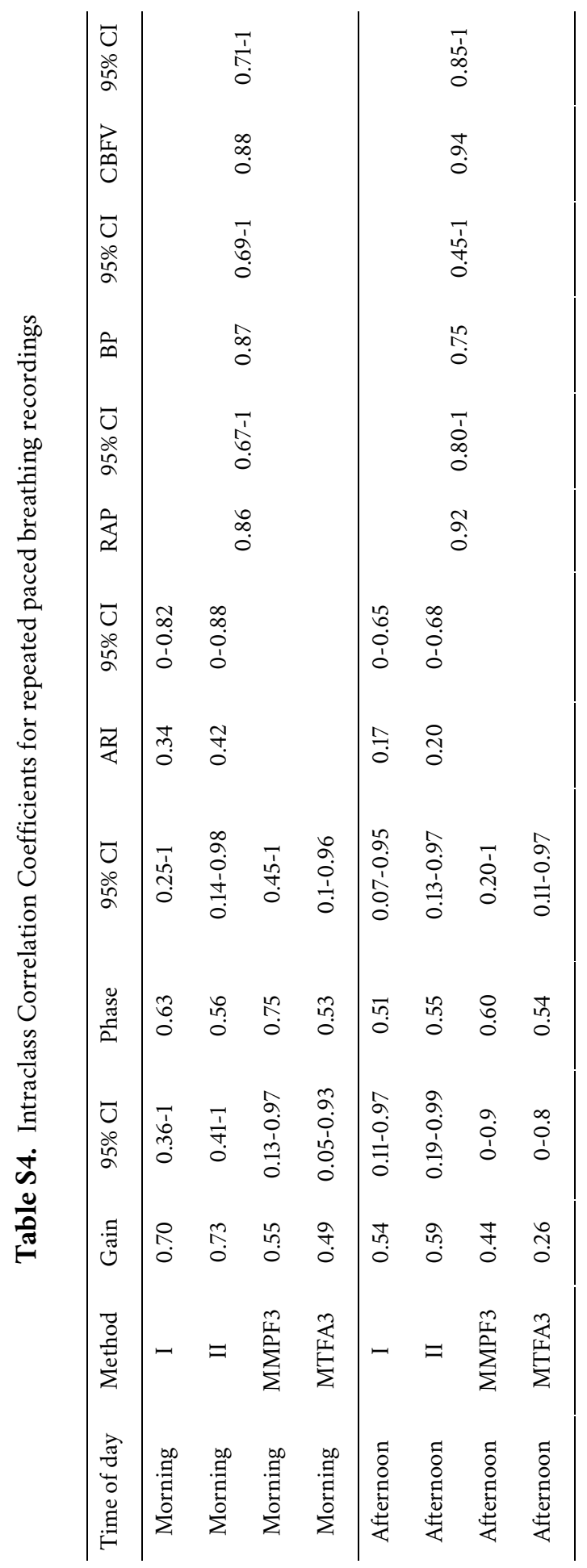


From the analysis of transfer function dCA parameters it becomes clear that ICC values are very low, meaning that $\mathrm{dCA}$ reproducibility is poor. From the dCA parameters ARI has the lowest ICC. Because of the poor ICC's and the low number of subjects in this study $95 \%$ confidence intervals for ICC are very wide and no significant differences can be found between different analysis methods, time-of-day, or breathing strategies. Earlier, Reinhard et al compared spontaneous versus paced breathing and could not demonstrate significant differences between both methods [26]. We do find significant differences for phase and ARI parameter values comparing both breathing methods. ARI is significantly higher in paced compared to spontaneous breathing in all but method I in the afternoon.

In order to detect impaired cerebral autoregulation during spontaneous breathing dCA phase should, based on our data, be less than 0.4 rad (criterion: mean minus 2 standard deviations). For MMPF the criterion for dCA impairment is a phase value less than $0.2 \mathrm{rad}$ for component 3 and 0.3 for component 4 . Due to the large inter- and intrasubject variability, the application of the different methods for the evaluation of dCA, although in good agreement, has limited value for the individual healthy subject. Whether this is also true for diseased populations remains to be investigated.

The ICC's we found for the MMPF method for phase are comparable with the reported values from $\mathrm{Hu}$ et al [11]. Only for paced breathing MMPF has a higher, although not significant, ICC compared to conventional transfer function analysis. In contrast with Hu et al we found better ICC for ARI, whereas significance in this case cannot be determined. Their low ICC value maybe explained by the fact that $\mathrm{Hu}$ et al calculate ARI by applying the Tiecks model only on short data segments, whereas we based the ARI on the estimated transfer function from the recording period of 15 minutes. We also report here ICC values for MMPF gain and the suggested MTFA. For gain MMPF seems to do better than the transfer function analysis, but the MTFA analysis does not seem to be an improvement. 


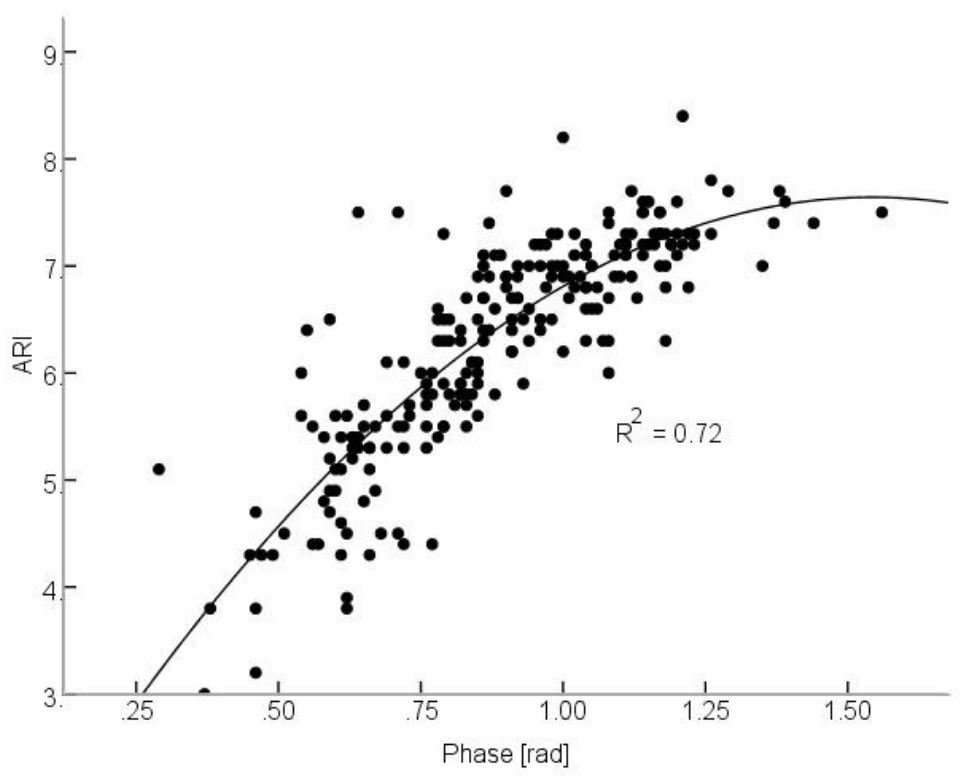

Figure 5. Relationship between index of autoregulation (ARI) and phase during spontaneous breathing for method II.

Recently, Czosnyka et al showed good correlation $(\mathrm{R}=0.62)$ between ARI and the time correlation based cerebral autoregulation method $\mathrm{Mx}$ [9]. We calculated ARI from the step response of our transfer function. Figure 5 shows the correlation between ARI and phase that was not shown before, which is even better $\left(\mathrm{R}^{2}=0.72\right)$. However, these correlations do not imply that dCA parameters reproduce well. Our low ICC values for ARI are comparable to the findings of Brodie et al while our ICC's for RAP show much higher values (ranging from 0.86 to 0.92 versus $0.70-0.75$ ) [6]. The reason for this difference is not clear. Brodie estimates RAP from the first harmonic of BP and CBFV [1], whereas we performed linear regression between BP and CBFV waves [21]. The possible influence of the analysis method with regard to this needs to be elaborated further. The high ICC values for reproducibility of RAP are promising and could open directions to a more robust parameter to estimate dCA. Although RAP in itself is not a measure of $\mathrm{dCA}$, the relation of variations in RAP in relation with ABP changes may be worthwhile to investigate.

Only in nine of the 19 subjects end-tidal $\mathrm{pCO}_{2}$ was recorded. Therefore, no results could be given for the whole group. As was shown by Reinhard et al [26], especially during paced breathing, where the subjects are likely to increase their tidal volumes influence of $\mathrm{pCO}_{2}$ can be seen. $\mathrm{pCO}_{2}$ drops after the start of paced 
breathing resulting in lower CBFV. From our results no benefits arise in favour of paced breathing versus spontaneous breathing.

In this study no impact of the different signal processing approaches on dCA parameters was found. It is shown that gain and phase are very robust under the different analysis methods evaluated and there is no need for prescribing a specific one of these signal-processing regimes. The threshold for significant coherence is not a fixed value, but depends on the degrees of freedom of each approach. Based on reproducibility results no preference can be made for morning versus afternoon measurements or for spontaneous versus paced breathing. Poor reproducibility of dCA parameters remains a major issue hampering the clinical applicability in individual patients. Future research should be directed towards less variable dCA parameters. RAP could be a promising parameter to further explore in developing methods for estimating dCA.

In conclusion, quantification of dCA does not favour TFA versus MMPF in terms of reproducibility, which is poor for both gain and phase. Neither does it favour as a stimulus spontaneous versus paced breathing and morning versus afternoon day-time recording.

\section{Acknowledgements}

The authors thank Prof. R.B. Panerai (University of Leicester, UK) for his valuable contributions to the manuscript. 


\section{Appendix}

The threshold for significant coherence depends on the degrees of freedom in the spectral analysis [12]. For sufficiently large degrees of freedom, the estimated coherence can be approximated by a $\chi^{2}$ distribution. Thus, equation A.1 merely applies an F-test with 2 and $\nu$ - 2 degrees of freedom,

$$
\gamma_{\min }^{2}=\frac{2 F_{2, v-2}(\alpha)}{v-2+2 F_{2, v-2}(\alpha)}
$$

where $\gamma_{\text {min }}^{2}$ is the minimum level above which the squared coherence significantly differs from zero. With equation A.1 the coherence threshold for each combination can be calculated. Others [26] used the following calculation of the coherence threshold: $\gamma_{\min }^{2}=1-\alpha^{\frac{2}{v-2}}$ [5]. This results in exactly the same coherence thresholds as when applying the F-test criterion.

The degrees of freedom are determined by the parameters used for spectral estimation and can be divided into two parts, one for the spectral averaging and one for the spectral smoothing. The total number of degrees of freedom for the combinations is the product of both numbers of degrees of freedom. The degrees of freedom for the spectral averaging is equal to the number of data segments and are proportional to $\mathrm{N} / \mathrm{L}$, where $\mathrm{N}$ is the total numbers of samples in the recording and $\mathrm{L}$ is the number of samples per segment.

For a 15 -minute period this means that with $\mathrm{L}=256 \mathrm{~N} / \mathrm{L} \approx 17.6$, resulting in $\nu=17$. For a 5-minute period this results in $v=5$ degrees of freedom. For combinations III and IV, $\mathrm{L}=2048$ and so $\mathrm{N} / \mathrm{L} \approx 2.2$ resulting in $\nu=2$ degrees of freedom.

For the degrees of freedom for the spectral smoothing the triangular smoothing window defined in equation A.2 needs to be taken into account.

$$
W_{i}=\frac{1}{h+1}-\frac{1}{(h+1)^{2}}|i|
$$

The degrees of freedom of this triangular smoothing window can be derived from equation A.3.

$$
v=\frac{2}{\sum_{i=-h}^{h} W_{i}^{2}}
$$

and for the different window widths the degrees of freedom are listed in table A1. 
Reproducibility of dynamic cerebral autoregulation

Table A1. Degrees of freedom for different smoothing window widths.

\begin{tabular}{ccc}
\hline window width & $\mathrm{h}$ & degrees of freedom \\
\hline 3 & 1 & 5.3 \\
31 & 15 & 47.9 \\
\hline
\end{tabular}

The results of the calculations for the total degrees of freedom and the resulting minimum squared coherence levels are shown in table A2.

Table A2. Total degrees of freedom and coherence thresholds for four combinations.

\begin{tabular}{|c|c|c|c|c|}
\hline $\begin{array}{l}\text { Combinati- } \\
\text { on }\end{array}$ & $\begin{array}{l}\text { Degrees } \\
\text { of } \\
\text { freedom } \\
\text { spectral } \\
\text { averaging }\end{array}$ & $\begin{array}{l}\text { Degrees of } \\
\text { freedom } \\
\text { spectral } \\
\text { smoothing }\end{array}$ & $\begin{array}{c}\text { Total } \\
\text { degrees of } \\
\text { freedom }\end{array}$ & $\begin{array}{l}\text { Squared Coheren- } \\
\text { ce threshold }\end{array}$ \\
\hline
\end{tabular}

\section{$15 \mathrm{~min}$}

\begin{tabular}{lcccc} 
I, II & 17 & 5.3 & 90 & 0.06 \\
III, IV & 2 & 47.9 & 96 & 0.06 \\
\hline $\mathbf{5}$ min & & & & \\
I, II & 5 & 5.3 & 27 & 0.2 \\
\hline
\end{tabular}




\section{References}

1. Aaslid R, Lash SR, Bardy GH, Gild WH, and Newell DW. Dynamic pressure-flow velocity relationships in the human cerebral circulation. Stroke 34: 1645-1649, 2003.

2. Aaslid R, Lindegaard KF, Sorteberg W, and Nornes H. Cerebral autoregulation dynamics in humans. Stroke 20: 45-52, 1989.

3. Ainslie PN, Murrell C, Peebles K, Swart M, Skinner MA, Williams MJ, and Taylor RD. Early morning impairment in cerebral autoregulation and cerebrovascular $\mathrm{CO} 2$ reactivity in healthy humans: relation to endothelial function. Exp Physiol 92: 769-777, 2007.

4. Blaber AP, Bondar RL, Stein F, Dunphy PT, Moradshahi P, Kassam MS, and Freeman R. Transfer function analysis of cerebral autoregulation dynamics in autonomic failure patients. Stroke 28: 1686-1692, 1997.

5. Brockwell PJ, and Davis RA. Time series: Theory and Methods. New York: Springer, 1991.

6. Brodie FG, Atkins ER, Robinson TG, and Panerai RB. Reliability of dynamic cerebral autoregulation measurement using spontaneous fluctuations in blood pressure. Clin Sci (Lond) 116: 513-520, 2009.

7. Claassen JA, Levine BD, and Zhang R. Dynamic cerebral autoregulation during repeated squat-stand maneuvers. J Appl Physiol 106: 153-160, 2009.

8. Cronbach LJ, Gleser GC, Nanda H, and Rjaratnam N. The dependability of behavioral measurements: Theory of generalizability for scores and profiles. New York: Wiley, 1972.

9. Czosnyka M, Smielewski P, Lavinio A, Pickard JD, and Panerai R. An assessment of dynamic autoregulation from spontaneous fluctuations of cerebral blood flow velocity: a comparison of two models, index of autoregulation and mean flow index. Anesth Analg 106: 234-239, table of contents, 2008.

10. Diehl RR, Linden D, Lucke D, and Berlit P. Phase relationship between cerebral blood flow velocity and blood pressure. A clinical test of autoregulation. Stroke 26: 1801-1804, 1995.

11. Hu K, Peng CK, Czosnyka M, Zhao P, and Novak V. Nonlinear assessment of cerebral autoregulation from spontaneous blood pressure and cerebral blood flow fluctuations. Cardiovasc Eng 8: 60-71, 2008.

12. Koopmans LH. The Spectral Analysis of Time Series. San Diego, California: Academic Press, 1995. 
13. Liau BY, Yeh SJ, Chiu CC, and Tsai YC. Dynamic cerebral autoregulation assessment using chaotic analysis in diabetic autonomic neuropathy. Med Biol Eng Comput 46: 1-9, 2008.

14. Lo MT, Hu K, Liu Y, Peng CK, and Novak V. Multimodal Pressure Flow Analysis: Application of Hilbert Huang Transform in Cerebral Blood Flow Regulation. EURASIP J Appl Signal Processing 2008: 785243, 2008.

15. Lo MT, Novak V, Peng CK, Liu Y, and Hu K. Nonlinear phase interaction between nonstationary signals: a comparison study of methods based on Hilbert-Huang and Fourier transforms. Phys Rev E Stat Nonlin Soft Matter Phys 79: 061924, 2009.

16. Mahony PJ, Panerai RB, Deverson ST, Hayes PD, and Evans DH. Assessment of the thigh cuff technique for measurement of dynamic cerebral autoregulation. Stroke 31: 476-480, 2000.

17. Mitsis GD, Poulin MJ, Robbins PA, and Marmarelis VZ. Nonlinear modeling of the dynamic effects of arterial pressure and $\mathrm{CO} 2$ variations on cerebral blood flow in healthy humans. IEEE Trans Biomed Eng 51: 1932-1943, 2004.

18. Muller T, Reinhard M, Oehm E, Hetzel A, and Timmer J. Detection of very low-frequency oscillations of cerebral haemodynamics is influenced by data detrending. Med Biol Eng Comput 41: 69-74, 2003.

19. Newell DW, Aaslid R, Lam A, Mayberg TS, and Winn HR. Comparison of flow and velocity during dynamic autoregulation testing in humans. Stroke 25: 793-797, 1994.

20. Novak V, Yang AC, Lepicovsky L, Goldberger AL, Lipsitz LA, and Peng CK. Multimodal pressure-flow method to assess dynamics of cerebral autoregulation in stroke and hypertension. Biomed Eng Online 3: 39, 2004.

21. Panerai RB. The critical closing pressure of the cerebral circulation. Med Eng Phys 25: 621-632, 2003.

22. Panerai RB, Dawson SL, and Potter JF. Linear and nonlinear analysis of human dynamic cerebral autoregulation. Am J Physiol 277: H1089-1099, 1999.

23. Panerai RB, Eames PJ, and Potter JF. Variability of time-domain indices of dynamic cerebral autoregulation. Physiol Meas 24: 367-381, 2003.

24. Paulson OB, Strandgaard S, and Edvinsson L. Cerebral autoregulation. Cerebrovasc Brain Metab Rev 2: 161-192, 1990.

25. Portney LG, and Watkins MP. Foundations of Clinical Research:Applications to Practice. Norwalk, Connecticut: Prentice Hall, 1999. 
26. Reinhard M, Muller T, Guschlbauer B, Timmer J, and Hetzel A. Transfer function analysis for clinical evaluation of dynamic cerebral autoregulation--a comparison between spontaneous and respiratory-induced oscillations. Physiol Meas 24: 27-43, 2003.

27. Simpson DM, Panerai RB, Ramos EG, Lopes JM, Marinatto MN, Nadal J, and Evans DH. Assessing blood flow control through a bootstrap method. IEEE Trans Biomed Eng 51: 1284-1286, 2004.

28. Streiner DL, and Norman GR. Health Measurement Scales: A Practical Guide to Their Development and Use. Oxford: Oxford University Press, 1995.

29. Tarvainen MP, Ranta-Aho PO, and Karjalainen PA. An advanced detrending method with application to HRV analysis. IEEE Trans Biomed Eng 49: 172-175, 2002.

30. Tiecks FP, Lam AM, Aaslid R, and Newell DW. Comparison of static and dynamic cerebral autoregulation measurements. Stroke 26: 10141019, 1995.

31. Timmer J, Lauk M, and Deuschl G. Quantitative analysis of tremor time series. Electroencephalogr Clin Neurophysiol 101: 461-468, 1996.

32. Welch PD. The use of fast Fourier transform for the estimation of power spectra: a method based on time averaging over short, modified periodograms. IEEE Trans Audio Electroacoust 15: 70-73, 1967.

33. Zhang R, Zuckerman JH, Giller CA, and Levine BD. Transfer function analysis of dynamic cerebral autoregulation in humans. Am J Physiol 274: H233-241, 1998. 


\section{Chapter 3}

Dynamic cerebral autoregulation and cerebrovascular reactivity: a comparative study in lacunar infarct patients

E D Gommer, J Staals, R J van Oostenbrugge, J Lodder, W H Mess and J P H Reulen. Dynamic cerebral autoregulation and cerebrovascular reactivity: a comparative study in lacunar infarct patients.

Physiol Meas. 2008 Nov;29(11):1293-303. 


\section{Abstract}

The major purpose of this study was to simultaneously evaluate dCA before and shortly after cerebral vasodilatation evoked by infusion of acetazolamide (ACZ). It was questioned if, and to what degree dCA was changed after ACZ infusion. Using $15 \mathrm{mg} / \mathrm{kg}$ ACZ infusion cerebrovascular reactivity (CVR) was assessed in 29 first ever lacunar stroke patients $(19 \mathrm{M} / 10 \mathrm{~F})$. During the CVR-test, the electrocardiogram, non-invasive finger arterial blood pressure (ABP) and middle cerebral artery blood flow velocity (CBFV) were recorded. DCA based on spontaneous blood pressure variations was evaluated in 24 subjects by linear transfer function analysis. Squared coherence, gain and phase angle in the frequency range of autoregulation $(0.04-0.16 \mathrm{~Hz})$ were compared before and after ACZ infusion. After ACZ infusion, median phase angle decreased significantly $(\mathrm{p}<0.005$ Wilcoxon) to $0.77 \mathrm{rad}$ compared to a pre-test baseline value of $1.05 \mathrm{rad}$, indicating less efficient dCA due to ACZ. However, post-test phase values are still mostly within the normal range. Poor and statistically non-significant correlations were found between CVR and absolute dCA phase angle.

It can be concluded that CVR testing with body weight adjusted infusion of ACZ lowers dCA performance but by no means exhausts dCA, suggesting that in this way maximal CVR is not determined. Characterizing dCA based on transfer function analysis of ABP to CBFV needs no provocation and adverse patient effects are minimal. The poor correlation between CVR and dCA phase angle is in support of an interpretation that CVRand dCA study different mechanisms of cerebrovascular control. 


\section{Introduction}

Cerebral autoregulation is the control mechanism that compensates for fluctuations in cerebral perfusion pressure and keeps the cerebral blood flow relatively constant in correspondence with the metabolic need of the brain $[1,22,26]$. The response to vasodilatory stimuli such as $\mathrm{CO}_{2}$ is traditionally used to evaluate and quantify what is called cerebrovascular reactivity (CVR) or vasomotor reactivity. CVR can also be assessed by the administration of acetazolamide (ACZ) [4, 27], which reversibly dilates the cerebral arteriolae and therefore increases cerebral blood flow. The exact mechanism by which ACZ increases cerebral blood flow remains unclear $[12,17,35]$. Using transcranial Doppler sonography the increase in cerebral blood flow can be shown by determining the cerebral blood flow velocity $(\mathrm{CBFV})$ in the main stem of the middle cerebral artery (MCA). Based on the assumption that the diameter of the MCA main stem is constant and vaso-

dilatation only occurs more peripherally, $\mathrm{CBFV}$ is proportional to cerebral blood flow. CVR is calculated as the percentage increase in CBFV after the administration of ACZ. So, the test results in a single value quantifying the cerebrovascular reactivity to a pharmacological stimulus.

Several methods are developed to test dynamic cerebral autoregulation (dCA) with and without induced blood pressure changes. In the former a sudden stepwise increase in arterial blood pressure (ABP) evokes a cerebral flow response. This response typically is characterized by a few seconds lasting increase followed within approximately 10 seconds by a relatively slow return to the base level. This response can be interpreted as a kind of step-response and is quantified by the so-called autoregulatory index [34]. Another approach to evaluate $\mathrm{dCA}$ is based on transfer function analysis of the presumed linear control system with $\mathrm{ABP}$ as the input signal and the CBFV in the MCA as the output signal [3, 36]. By calculating the auto and cross spectra of and between ABP and CBFV, the transfer function and its gain and phase spectrum are estimated. Furthermore, coherence indicates the strength of the linear relation between ABP and CBFV. The relationship between ACZ-induced CVR and simultaneous dCA has not been studied before. This comparison is clinically relevant since it has been suggested that dCA and CVR might test different properties of the cerebrovascular control system $[9,15,26,30]$. Only a thorough understanding of the relations between the outcome of CVR and simultaneous dCA testing may enable proper interpretation of published data across modalities.

As part of a research project into different aspects of lacunar stroke we were able to study simultaneously dCA and CVR in patients with lacunar stroke thought to be due to cerebral small vessel disease [8]. The simultaneous investigation of dCA 
and CVR in such a patient group seems to be relevant since multiple studies [13, $14,16,18,19]$ have shown impaired cerebral autoregulation in patients with lacunar stroke.

Major aim of our study into cerebrovascular function was to evaluate dCA parameters before and after ACZ infusion to quantify CVR. A sub question was whether dCA is reduced to a non-functional level after ACZ infusion at a dosage normally used for routine diagnostics and expected to test maximal cerebrovascular reserve capacity. Eventually, it was questioned whether dCA parameters relate to CVR results.

\section{Methods}

\section{Subjects and measurements}

The study group consisted of 29 subjects (19M/10F, median age 67 ranging from 40 to $80 \mathrm{yrs}$ ) who suffered a first ever lacunar stroke. Lacunar stroke is defined as an acute stroke syndrome with an MRI finding compatible with the patient's clinical status. This in combination with either a T2-weighted hyperintense subcortical lesion smaller than $20 \mathrm{~mm}$ in diameter or an acute stroke syndrome compatible with one of the lacunar syndromes in the absence of such MRI lesion [2]. The Institutional Review Board approved the study and all subjects gave written informed consent. Patients with a stenosis of the common or internal carotid artery or the MCA of more than 50\%, as detected by extracranial or transcranial color-coded duplex, were excluded. The electrocardiogram (ECG) and non-invasive ABP were measured using a Task Force Monitor (CN Systems ${ }^{\circledR}$, Austria). A transcranial Doppler system (Multidop X4, DWL ${ }^{\circledR}$, Sipplingen, Germany) was used to measure CBFV in the main stem of both the right and left MCA. Two $2 \mathrm{MHz}$-probes were held in position by a special frame. Patients were in supine position during all recordings.

Dynamic cerebral autoregulation was investigated before and after the administration of ACZ, which was adjusted to body weight (15 mg/kg). The ACZ was dissolved in water in a concentration of $100 \mathrm{mg} / \mathrm{ml}$. The infusion was performed at a speed of $1 \mathrm{ml} / \mathrm{min}$. Before the $\mathrm{ACZ}$ infusion was started, a measurement lasting at least five minutes was performed. Then $A C Z$ was administered which on average lasted 10 minutes. Upon finishing ACZ infusion the measurement was continued for at least another 10 minutes until a steady state was reached. DCA data were analyzed separately for pre- and post-ACZ infusion. 
Except for 1 patient all were on statin treatment and about 50\% of the patients were on some kind of antihypertensive treatment. Medications were not discontinued during the testing phase.

The measurements were performed in the non-acute stroke phase, i.e. 6 weeks to 6 months post stroke (median 93, ranging from 50 to 190 days after stroke).

\section{Data analysis}

An automatic algorithm in the Task Force Monitor detected R-waves from the ECG and between every two R-waves the diastolic, systolic and mean values of the blood pressure waveform was determined. Correspondingly the peaksystolic, end-diastolic and mean CBFV values were determined. The beat-to-beat mean values of $\mathrm{ABP}$ and $\mathrm{CBFV}$ were exported to Matlab and resampled at $5 \mathrm{~Hz}$ using spline-interpolation [24]. From the ABP the mean value was subtracted and the CBFVs were normalized with respect to the mean. This resulted in zeromean signals suited for spectral analysis to estimate the transfer function. The transfer function was calculated by

$H(f)=\frac{S_{x y}(f)}{S_{x x}(f)}$

where $S_{x x}(f)$ is the auto spectrum of changes in arterial blood pressure and $S_{x y}(f)$ is the cross-spectrum between the ABP- and CBFV-signals. The transfer function magnitude $|H(f)|$ and phase spectrum $\Phi(f)$ were derived from the real part $H_{R}(f)$ and imaginary part $H_{I}(f)$ of the complex transfer function as

$$
\begin{aligned}
& |H(f)|=\sqrt{\left[H_{R}(f)\right]^{2}+\left[H_{I}(f)\right]^{2}} \\
& \Phi(f)=\arctan \frac{H_{I}(f)}{H_{R}(f)}
\end{aligned}
$$

The squared coherence function $\gamma^{2}(f)$ was estimated by

$$
\gamma^{2}(f)=\frac{\left|S_{x y}(f)\right|^{2}}{S_{x x}(f) S_{y y}(f)}
$$

where $S_{y y}(f)$ is the auto spectrum of changes in cerebral blood flow velocity. The squared coherence reflects the strength of the linear relationship between $A B P$ and CBFV for each frequency on a scale from 0 to 1.

For the estimation of the transfer function ensemble averaging was used with half overlapping windows with a length of 256 data points. With a $5 \mathrm{~Hz}$ sampling rate this resulted in 51.2 seconds long data epochs. The smoothness priors approach [33] was used to remove the very low frequency trend. A hanningwindow was applied on each data epoch. The spectrum was smoothed with a triangular window of 3 points wide corresponding to a bandwidth of $0.04 \mathrm{~Hz}$. 
From the resulting transfer functions, coherence, phase angle $\Phi$ and gain were calculated in the low frequency (LF) range from $0.04-0.16 \mathrm{~Hz}$ where autoregulation is active and in the high frequency $(\mathrm{HF})$ range from $0.2-0.4 \mathrm{~Hz}$ which is too fast for autoregulation to work.

Cerebrovascular reactivity was quantified by equation 5 . In the baseline, before ACZ infusion, the average of CBFV is taken from a visually inspected artefact free episode $\left(\mathrm{CFBV}_{\text {base }}\right)$. After $\mathrm{ACZ}$ infusion another artefact free episode of $\mathrm{CBFV}$ is averaged to determine $\mathrm{CBFV}_{\mathrm{ACZ}}$.

$$
C V R=\frac{C B F V_{A C Z}-C B F V_{\text {base }}}{C B F V_{\text {base }}} \bullet 100 \%
$$

\section{Statistical analysis}

The Wilcoxon signed ranks test was used to compare the calculated parameters from the transfer function before and after $\mathrm{ACZ}$ administration. Linear regression was used to evaluate the relation between CVR and dCA parameters.

\section{Results}

It was feasible to determine CVR in all 29 subjects; however, due to a decreased ultrasound permeability of the transtemporal bone window, dCA evaluation was not possible in five subjects. They were excluded from this study. In the remaining 24 subjects, data from one recording side (4 left, 1 right MCA) were also excluded for further analysis because of excessive CBFV measuring artefacts. In these five subjects parameter values derived from the other, artefact-free, recording side were used. Statistical testing of the remaining 19 left and right side derived parameters showed no significant side differences. Therefore, parameter values of the left and right MCA were averaged for each of these 19 individuals. So, for each of the 24 subjects a complete parameter data set for the evaluation of CVR and dCA was created.

Median group values and corresponding 95\% confidence intervals as computed for the squared coherence, gain and phase angle before and after ACZ infusion for the low and high frequency band are given in table 1. Also, the mean heart rate, mean $\mathrm{ABP}$ and mean $\mathrm{CBFV}$ with $95 \%$ confidence intervals before and after ACZ infusion as well as the CVR is shown. Note, that the administration of 15 $\mathrm{mg} / \mathrm{kg} \mathrm{ACZ}$ had no effect on mean heart rate and mean ABP. 
Table 1. Median and 95\% confidence interval of measured values and calculated dCA

\begin{tabular}{|c|c|c|}
\hline & Before ACZ & After ACZ \\
\hline mean heart rate $[\mathrm{bpm}]$ & $68(64-74)$ & $69(65-73)$ \\
\hline mean ABP [mmHg] & $102(96-111)$ & $101(93-113)$ \\
\hline mean CBFV $[\mathrm{cm} / \mathrm{s}]$ & $41(37-46)$ & $58(56-70)^{*}$ \\
\hline Squared coherence LF & $0.38(0.29-0.42)$ & $0.51(0.39-0.56)^{*}$ \\
\hline Gain LF $[\% / \mathrm{mmHg}]$ & $1.3(1.1-1.4)$ & $1.2(0.85-1.2)^{\dagger}$ \\
\hline Phase angle LF [rad] & $1.05(0.97-1.12)$ & $0.77(0.58-0.84)^{*}$ \\
\hline Squared coherence HF & $0.44(0.32-0.50)$ & $0.45(0.37-0.53)$ \\
\hline Gain HF $[\% / \mathrm{mmHg}]$ & $1.7(1.4-2.1)$ & $1.3(1.1-1.6)^{\dagger}$ \\
\hline Phase angle HF [rad] & $0.14(0.04-0.23)$ & $0.11(0.02-0.22)$ \\
\hline CVR [\%] & & $67(56-74)$ \\
\hline
\end{tabular}

${ }^{*} \mathrm{p}<0.005,{ }^{\dagger} \mathrm{p}<0.05$ Wilcoxon signed ranks test

Before ACZ infusion, the median phase angle in the low frequency range $\Phi_{\mathrm{LF}}$ was $1.05 \mathrm{rad}$ indicating normal dCA as compared to reported [28] controlvalues $(0.84$ $\pm 0.26 \mathrm{rad})$. After ACZ infusion $(0.77 \mathrm{rad})$, there was a significant decrease $(\mathrm{p}<0.005)$. It is noteworthy, that despite these changes at the end of the test $\Phi_{\mathrm{LF}}$ values still were within the normal range.

After ACZ administration also the squared coherence in the low frequency range was significantly increased as compared to before ACZ infusion, whereas the gain in both the low and high frequency range decreased significantly after ACZ infusion $(\mathrm{p}<0.05)$.

Figure 1 shows the group averaged phase angle values as a function of frequency before and after ACZ infusion. For frequencies exceeding $0.2 \mathrm{~Hz}$ there is no significant difference between the two periods. On the contrary, for the low frequency range a phase angle decrease due to ACZ-infusion can clearly be seen. In our group of 24 patients, median CVR was $67 \%$. In figure $2 \Phi_{\mathrm{LF}}$ before and after ACZ infusion are plotted against CVR for each subject, including linear regression lines and their squared linear correlation coefficients. Although the correlations are rather weak, it appears that after ACZ-infusion $\Phi_{\mathrm{LF}}$ decreases with increasing CVR value. 


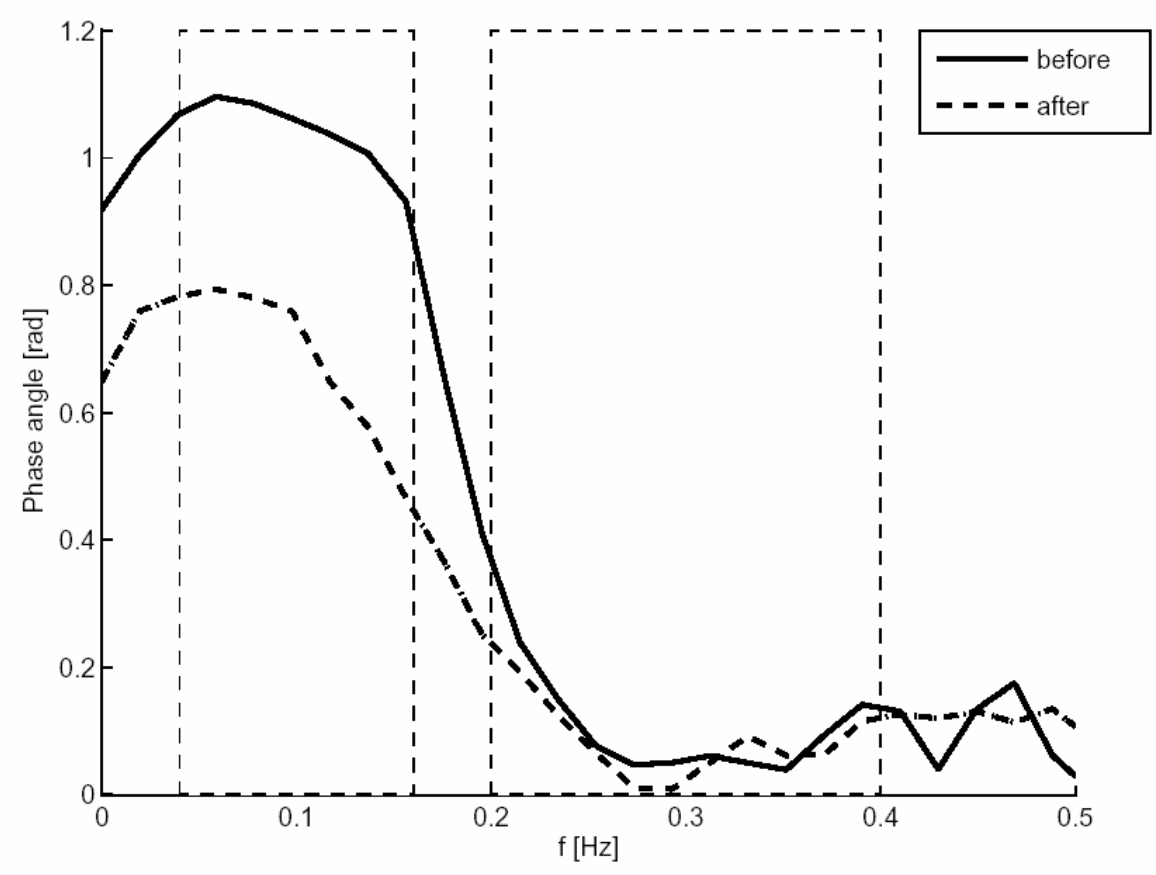

Figure 1. Group-averaged spectral phase plot before (solid) and after (dashed) acetazolamide infusion. Marked by the dashed boxes are the low frequency and high frequency ranges.

\section{Discussion}

\section{Dynamic cerebral autoregulation}

In this study cerebral autoregulation parameters were studied in first-ever lacunar infarct patients. The phase angle in the low frequency range $\left(\Phi_{\mathrm{LF}}\right)$ is regarded to be the dominant and most relevant parameter to quantify dCA [23]. Theoretically, $\Phi_{\mathrm{LF}}$ may range from $-\pi$ to $+\pi$ radians. In human, positive $\Phi_{\mathrm{LF}}$ around 0.9 indicates normal autoregulation. Before ACZ infusion, baseline dCA has a median $\Phi_{\mathrm{LF}}$ of 1.05 radians which is in the normal range as shown in control subjects by Reinhard et al [28](0.84 $\pm 0.26 \mathrm{rad})$ and Immink et al [13] $(1.0 \pm 0.3 \mathrm{rad})$. As opposed to our findings, Immink and co-workers showed in the same study for lacunar infarct patients reduced $\Phi_{\mathrm{LF}}(0.57 \pm 0.3 \mathrm{rad})$. Two facts may explain the discrepancy between Immink's findings and ours. First, in Immink's study dCA was assessed within 72 hours in the acute post-infarct time span, whereas we performed our measurements between one and two months post-infarct. Second, in the post-infarct period all our patients were treated with statins, a medication 
strategy not reported by Immink. Since statins are known to improve CVR [32] possibly by improving endothelial function, it is conceivable that statins also may improve or even normalize worsened dCA.

\section{Cerebrovascular reactivity}

The median CVR found in our study group is $67 \%$. Molina et al [19] found values of $65 \%$ in control subjects and 50\% in lacunar infarct patients, which are significantly lower. Other investigators have reported lower CVR normal values ranging from $36-52 \%[20,31]$. As such, this shows that our CVR results are, except for three patients (CVR=20, 28 and 29\%), in the normal range. Molina et al evaluated their infarct patients at least one month post-infarct. This leaves statin treatment the most plausible cause of normal CVR in $88 \%$ of our lacunar infarct patients one to two month post infarct. However, further study is needed to determine the contribution of the effect of statin treatment on CVR results.

Dumville et al [7] showed that in $\mathrm{CO}_{2}$ reactivity testing blood pressure monitoring is needed because the $\mathrm{CO}_{2}$ challenge also increased mean arterial blood pressure. $\mathrm{CO}_{2}$ and $\mathrm{ACZ}$ both have a potent systemic vasodilatory effect. In our study blood pressure was also monitored, but no effect of ACZ infusion on mean $\mathrm{ABP}$ was observed, ruling out systemic blood pressure increase as the cause of post-ACZ increased CBFV.

\section{Dynamic cerebral autoregulation and cerebrovascular reactivity testing}

This study firstly evaluates dCA parameters based on transfer function analysis in the course of CVR-testing with acetazolamide. The, as compared to baseline, decrease of the phase in the low frequency range from median 1.05 to $0.77 \mathrm{rad}$ after administration of $15 \mathrm{mg} / \mathrm{kg}$ ACZ indicates less efficient cerebral autoregulation. However, post ACZ infusion $\Phi_{\mathrm{LF}}$ values are still mostly (in $83 \%$ ) within normal range $[13,28]$. So, cerebral autoregulation is far from full exhaustion at least ten minutes after the end of infusion of ACZ. Reinhard et al [29] showed that in patients with ipsilateral ICA occlusion the phase angle in the MCA can even get below zero. It is therefore likely that phase angle values lower than those we obtained after ACZ administration indeed indicate a more disturbed cerebral autoregulation. It can be assumed that the evaluation of CVR only informs us about a fraction of the reserve capacity of the cerebral autoregulation system. 
Chapter 3

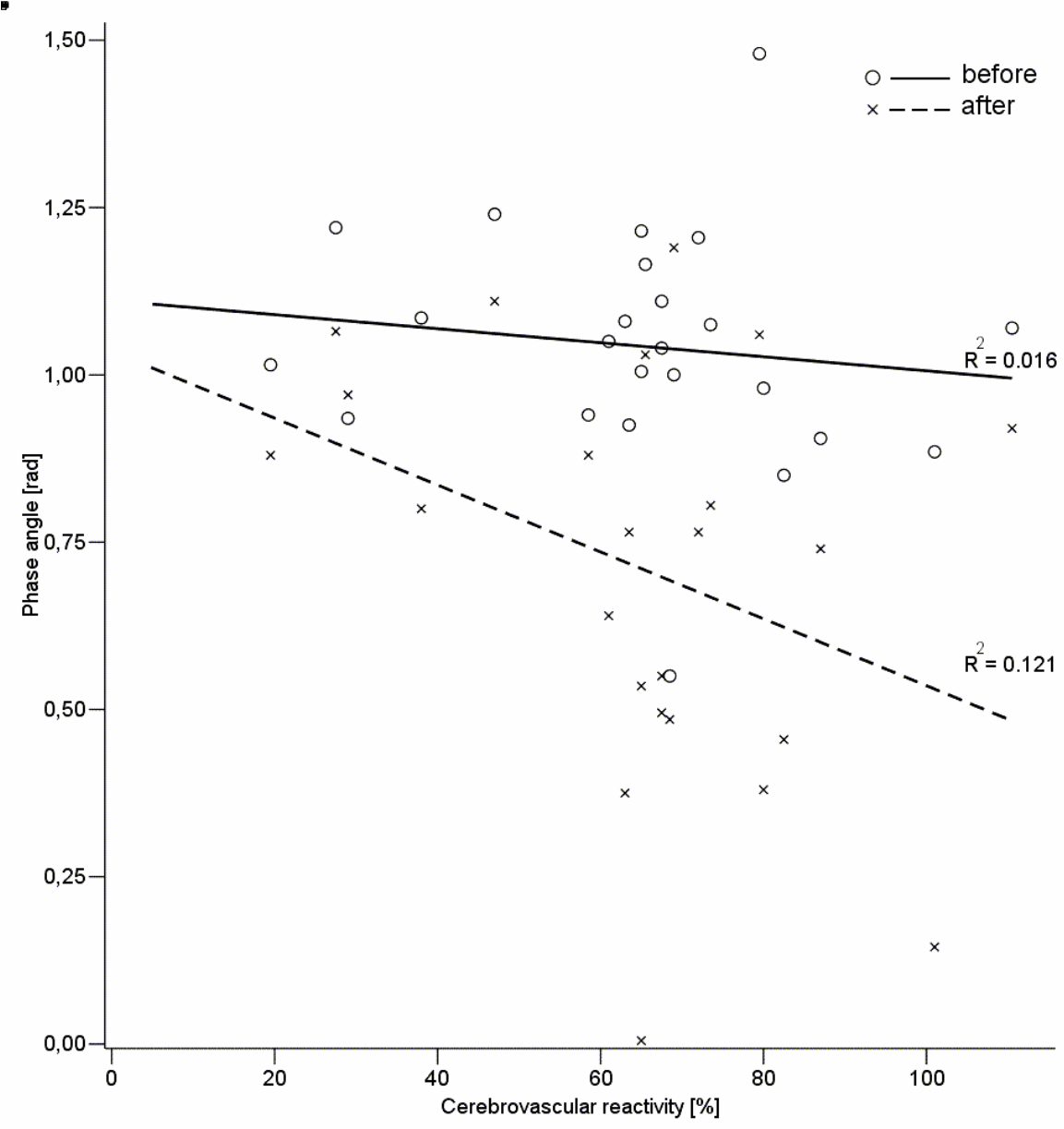

Figure 2. Dynamic cerebral autoregulation phase angle in the low frequency range versus cerebrovascular reactivity before ( $\mathrm{O}$ solid) and after $(\times$ dashed) acetazolamide infusion.

The question can be raised whether cerebral autoregulation still is intact to a large extent, because the dose of ACZ was insufficient for maximal vasodilatation. The dose-response relationship of ACZ has been investigated both in animal and human studies [5, 6, 21],[11]. From animal experiments, there is evidence that ACZ administration does evaluate only part of the autoregulatory capacity. In anesthetized rats it was shown that autoregulation was not exhausted after acetazolamide administration even at a dose of $21 \mathrm{mg} / \mathrm{kg}$. In humans, Grossmann et al [11] showed that with $18 \mathrm{mg} / \mathrm{kg}$ a higher cerebrovascular reactivity was measured than with $13 \mathrm{mg} / \mathrm{kg}$. Yet, based on the adverse effects, although re- 
versible, they concluded that the latter dose was the optimal choice for clinical testing. For standardized test conditions these authors strictly recommended weight-adjusted administration. In our study we applied $15 \mathrm{mg} / \mathrm{kg}$. A higher dose of ACZ would probably have further increased CBFV and further impaired cerebral autoregulation, but also would have increased adverse effects (only one of the patients tested in this study reported slight light-headedness) and is therefore not recommendable. So, CVR testing using ACZ administration with a dose of $15 \mathrm{mg} / \mathrm{kg}$ is not capable of determining the CVR to its full extent and should not be interpreted as such.

We not only observed a decrease of the phase angle after ACZ infusion but also a statistically significant $(\mathrm{p}<0.005)$ increase of the coherence in the autoregulation frequency band. Increased coherence indicates that the relation between $\mathrm{ABP}$ and CBFV becomes more linear. In other words, the waveform of the CBFV signal resembles more the waveform of the ABP signal. This is typical for increased autoregulation impairment since a well-functioning autoregulation prevents blood pressure variations being transferred to the CBFV. Increased coherence can be interpreted as worsened autoregulation [10], a finding which is in line with our $\Phi_{\mathrm{LF}}$ related findings.

\section{$d C A$ versus $C V R$}

CVR and dCA parameters show poor correlation. In figure $2 \Phi_{\mathrm{LF}}$ is plotted versus $\mathrm{CVR}$ for the pre-and post-ACZ infusion period. Although not significant, there seems to be a negative linear relation between CVR and $\Phi_{\mathrm{LF}}$ post ACZ infusion. The regression lines in this graph show that for subjects with low CVR the effect on the phase angle in the two stages is minimal whereas for high CVR the decrease in phase angle after acetazolamide infusion is more pronounced. Plotting (relative) phase changes against CVR did not show this more markedly. DCAgain is hardly influenced by the infusion of ACZ.

The negative correlation between phase angle and CVR suggests that increased cerebrovascular flow due to diameter increase of the arterioles decreases $\mathrm{dCA}$ performance. A comparison with a rubber hose comes up, where increasing the diameter diminishes the elasticity of the hose and by that the degree too which the diameter can be varied.

The exact mechanism by which ACZ increases cerebral blood flow remains unclear $[12,17,35]$. But, regardless of the cause of vasodilatation, at higher levels of cerebral blood flow the plateau in the static autoregulation curve is less wide (less autoregulated) [26]. Subjects with low CVR have only a small increase in $\mathrm{CBFV}$ and therefore the static autoregulation curve is not "flow" shifted and narrowed much. As a result, autoregulation remains intact. For subjects with high 
CVR the autoregulation curve is lifted and narrowed more, resulting in less effective autoregulation i.e. lowered phase angle. Concerning dCA gain, Panerai et al [25] showed $\mathrm{CO}_{2}$-effects resulted in lowered critical closing pressure in hypercapnic conditions whereas the resistance area product remained nearly unchanged. This might explain the slightly decreased dCA gain we found post versus pre-ACZ testing, since for the same change in $\mathrm{ABP}$ the percentage change in CBFV is less.

Many authors $[9,15,26,30]$ have stated that CVR and dCA are testing different cerebrovascular control properties. The increase in CBFV provoked by ACZ is commonly assumed to work via carbonic anhydrase inhibition resulting in perivascular acidosis leading to vasodilatation [17,35], although this is debated by others [12]. Conversely, dynamic cerebral autoregulation involves control of $\mathrm{CBFV}$ based on changes in cerebral perfusion pressure. However, it is also known that $\mathrm{pCO}_{2}$ levels influence properties of dCA [25].

In this study dCA parameters based on transfer function analysis were determined during a CVR-test. Our results illustrate the ambiguity of the relation between CVR and dCA testing, since on the one hand there was a clear-cut effect of CBFV-increase on dCA but on the other hand the correlation of the results of both tests was poor, which supports that at least partly different phenomena are studied. Further research is needed to examine the interaction between both phenomena.

Limitations of our study concern both selection of the kind of patients and the dCA method used. Regarding the first, this study was performed in first ever lacunar infarct patients. To investigate the influence of the infarct and the statin treatment a more extensive study in this patient group as well as in healthy subjects is recommended.

Regarding the method used to quantify dCA; it is based on transfer function analysis and depends on spontaneous blood pressure variations. This means that no external stimulus is delivered and no additional burden is put on to the patient. Yet, a possible limitation could be insufficient arterial blood pressure variability. The method requires sufficient variability in both blood pressure and cerebral blood flow velocity in the frequency range of interest. This becomes expressed in the coherence between blood pressure and flow, which in our case should exceed 0.1 in the dCA frequency band of interest. The coherence levels in this study did exceed this value. 


\section{Conclusions}

Weight adjusted infusion of ACZ to test CVR affects dCA parameters, but by no means exhausts dCA. It is therefore doubtful that maximal CVR is achieved this way. Statements about the function of cerebral blood flow control, like maximal reserve or reserve capacity, should always be interpreted in reference to the method that was used. Characterizing dCA based on transfer function analysis of $\mathrm{ABP}$ to $\mathrm{CBFV}$ changes has the advantage that no provocation is needed and adverse effects are minimal. The poor negative correlation between CVR and phase angle supports the notion that different mechanisms are involved, which interact in some way. DCA seems to depend on the "flow state" i.e. the overall "baseline" diameter of the peripheral cerebrovascular arterioles. Further studies should be directed to uncover the physiological mechanisms contributing to $\mathrm{dCA}, \mathrm{CVR}$ and their relationships. In particular interaction between CVR and dCA should be studied also in a control group.

\section{Acknowledgements}

The authors thank Prof. R.B. Panerai (University of Leicester, UK) for his valuable contributions to the manuscript.

\section{References}

1. Aaslid R, Lindegaard KF, Sorteberg W, and Nornes H. Cerebral autoregulation dynamics in humans. Stroke 20: 45-52, 1989.

2. Bamford J, Sandercock P, Jones L, and Warlow C. The natural history of lacunar infarction: the Oxfordshire Community Stroke Project. Stroke 18: 545-551, 1987.

3. Blaber AP, Bondar RL, Stein F, Dunphy PT, Moradshahi P, Kassam MS, and Freeman R. Transfer function analysis of cerebral autoregulation dynamics in autonomic failure patients. Stroke 28: 1686-1692, 1997.

4. Dahl A, Russell D, Rootwelt K, Nyberg-Hansen R, and Kerty E. Cerebral vasoreactivity assessed with transcranial Doppler and regional cerebral blood flow measurements. Dose, serum concentration, and time course of the response to acetazolamide. Stroke 26: 2302-2306, 1995.

5. Demolis P, Florence G, Thomas L, Tran Dinh YR, Giudicelli JF, Seylaz J, and Alkayed NJ. Is the acetazolamide test valid for quantitative assessment of maximal cerebral autoregulatory vasodilation? An experimental study. Stroke 31: 508-515, 2000. 
6. Derdeyn CP. Is the acetazolamide test valid for quantitative assessment of maximal cerebral autoregulatory vasodilation? Stroke 31: 2271-2272, 2000.

7. Dumville J, Panerai RB, Lennard NS, Naylor AR, and Evans DH. Can cerebrovascular reactivity be assessed without measuring blood pressure in patients with carotid artery disease? Stroke 29: 968-974, 1998.

8. Fisher CM. Lacunar strokes and infarcts: a review. Neurology 32: 871876, 1982.

9. Garnham J, Panerai RB, Naylor AR, and Evans DH. Cerebrovascular response to dynamic changes in pCO2. Cerebrovasc Dis 9: 146-151, 1999.

10. Giller CA, and Mueller M. Linearity and non-linearity in cerebral hemodynamics. Med Eng Phys 25: 633-646, 2003.

11. Grossmann WM, and Koeberle B. The dose-response relationship of acetazolamide on the cerebral blood flow in normal subjects. Cerebrovasc Dis 10: 65-69, 2000.

12. Hauge A, Nicolaysen G, and Thoresen M. Acute effects of acetazolamide on cerebral blood flow in man. Acta Physiol Scand 117: 233-239, 1983.

13. Immink RV, van Montfrans GA, Stam J, Karemaker JM, Diamant M, and van Lieshout JJ. Dynamic cerebral autoregulation in acute lacunar and middle cerebral artery territory ischemic stroke. Stroke 36: 2595-2600, 2005.

14. Isaka Y, Okamoto M, Ashida K, and Imaizumi M. Decreased cerebrovascular dilatory capacity in subjects with asymptomatic periventricular hyperintensities. Stroke 25: 375-381, 1994.

15. Lundar T, Lindegaard KF, Froysaker T, Aaslid R, Grip A, and Nornes H. Dissociation between cerebral autoregulation and carbon dioxide reactivity during nonpulsatile cardiopulmonary bypass. Ann Thorac Surg 40: 582-587, 1985.

16. Maeda H, Matsumoto M, Handa N, Hougaku H, Ogawa S, Itoh T, Tsukamoto Y, and Kamada T. Reactivity of cerebral blood flow to carbon dioxide in various types of ischemic cerebrovascular disease: evaluation by the transcranial Doppler method. Stroke 24: 670-675, 1993.

17. Maren TH. Carbonic anhydrase: chemistry, physiology, and inhibition. Physiol Rev 47: 595-781, 1967.

18. Matsushita K, Kuriyama Y, Nagatsuka K, Nakamura M, Sawada T, and Omae T. Periventricular white matter lucency and cerebral blood flow autoregulation in hypertensive patients. Hypertension 23: 565-568, 1994. 
19. Molina C, Sabin JA, Montaner J, Rovira A, Abilleira S, and Codina A. Impaired cerebrovascular reactivity as a risk marker for first-ever lacunar infarction: A case-control study. Stroke 30: 2296-2301, 1999.

20. Muller M, Voges M, Piepgras U, and Schimrigk K. Assessment of cerebral vasomotor reactivity by transcranial Doppler ultrasound and breath-holding. A comparison with acetazolamide as vasodilatory stimulus. Stroke 26: 96-100, 1995.

21. Nemoto EM, and Yonas H. Revisiting the question, "is the acetazolamide test valid for quantitative assessment of maximal cerebral autoregulatory vasodilation?" Stroke 32: 1234-1237, 2001.

22. Newell DW, Aaslid R, Lam A, Mayberg TS, and Winn HR. Comparison of flow and velocity during dynamic autoregulation testing in humans. Stroke 25: 793-797, 1994.

23. Panerai RB. Cerebral Autoregulation: From Models to Clinical Applications. Cardiovasc Eng 8: 42-59, 2008.

24. Panerai RB, Dawson SL, and Potter JF. Linear and nonlinear analysis of human dynamic cerebral autoregulation. Am J Physiol 277: H1089-1099, 1999.

25. Panerai RB, Deverson ST, Mahony P, Hayes P, and Evans DH. Effects of $\mathrm{CO} 2$ on dynamic cerebral autoregulation measurement. Physiol Meas 20: 265-275, 1999.

26. Paulson OB, Strandgaard S, and Edvinsson L. Cerebral autoregulation. Cerebrovasc Brain Metab Rev 2: 161-192, 1990.

27. Piepgras A, Schmiedek P, Leinsinger G, Haberl RL, Kirsch CM, and Einhaupl KM. A simple test to assess cerebrovascular reserve capacity using transcranial Doppler sonography and acetazolamide. Stroke 21: 1306-1311, 1990.

28. Reinhard M, Roth M, Guschlbauer B, Harloff A, Timmer J, Czosnyka M, and Hetzel A. Dynamic cerebral autoregulation in acute ischemic stroke assessed from spontaneous blood pressure fluctuations. Stroke 36:16841689, 2005.

29. Reinhard M, Roth M, Muller T, Czosnyka M, Timmer J, and Hetzel A. Cerebral autoregulation in carotid artery occlusive disease assessed from spontaneous blood pressure fluctuations by the correlation coefficient index. Stroke 34: 2138-2144, 2003.

30. Singhal S, and Markus HS. Cerebrovascular reactivity and dynamic autoregulation in nondemented patients with CADASIL (cerebral autosomal dominant arteriopathy with subcortical infarcts and leukoencephalopathy). J Neurol 252: 163-167, 2005. 
31. Sorteberg W, Lindegaard KF, Rootwelt K, Dahl A, Nyberg-Hansen R, Russell D, and Nornes H. Effect of acetazolamide on cerebral artery blood velocity and regional cerebral blood flow in normal subjects. Acta Neurochir (Wien) 97: 139-145, 1989.

32. Sterzer P, Meintzschel F, Rosler A, Lanfermann H, Steinmetz H, and Sitzer M. Pravastatin improves cerebral vasomotor reactivity in patients with subcortical small-vessel disease. Stroke 32: 2817-2820, 2001.

33. Tarvainen MP, Ranta-Aho PO, and Karjalainen PA. An advanced detrending method with application to HRV analysis. IEEE Trans Biomed Eng 49: 172-175, 2002.

34. Tiecks FP, Lam AM, Aaslid R, and Newell DW. Comparison of static and dynamic cerebral autoregulation measurements. Stroke 26: 10141019, 1995.

35. Vorstrup S, Henriksen L, and Paulson OB. Effect of acetazolamide on cerebral blood flow and cerebral metabolic rate for oxygen. J Clin Invest 74: 1634-1639, 1984.

36. Zhang R, Zuckerman JH, Giller CA, and Levine BD. Transfer function analysis of dynamic cerebral autoregulation in humans. Am J Physiol 274: H233-241, 1998. 
Chapter 4

Dynamic cerebral autoregulation in subjects with Alzheimer's disease, mild cognitive impairment and controls: evidence for increased peripheral vascular resistance with possible predictive value.

E D Gommer, E G H J Martens, P Aalten, E Shijaku, F R J Verhey, W H Mess, I H G B Ramakers and J P H Reulen. Dynamic cerebral autoregulation in subjects with Alzheimer's disease, mild cognitive impairment and controls: evidence for increased peripheral vascular resistance with possible predictive value.

J Alzheimers Dis. 2012; 30(4):805-13. 


\section{Abstract}

Cerebrovascular dysfunction plays a role not only in vascular causes of cognitive impairment but also in Alzheimer's disease (AD). We hypothesized that cerebral autoregulation is impaired in patients with $\mathrm{AD}$ compared to subjects with mild cognitive impairment (MCI) and controls. Dynamic cerebral autoregulation (dCA) was investigated in $17 \mathrm{AD}$ patients, $19 \mathrm{MCI}$ subjects and 20 controls (C). Groups were matched for age, sex and level of education.

Electrocardiogram and non-invasive finger arterial blood pressure were measured and transcranial doppler ultrasonography was used to measure cerebral blood flow velocity in right and left middle cerebral artery (MCA). Also cerebrovascular resistance index CVRi was computed. dCA in supine position was quantified based on spontaneous blood pressure variations by computation of the linear transfer function between arterial blood pressure and MCA cerebral blood flow velocity. dCA gain and phase were evaluated for different frequency bands. Results were also evaluated using a 3-parameter windkessel model (WKM).

CVRi was significantly higher in $\mathrm{AD}(2.9 \pm 0.2)$ compared to both MCI $(2.3 \pm 0.1$, $\mathrm{p}=0.02)$ and $\mathrm{C}(2.1 \pm 0.1 \mathrm{mmHgs} / \mathrm{cm}, \mathrm{p}=0.002)$. Five MCI patients who converted to AD during the course of the study also had higher CVRi compared to nonconverters ( $2.8 \pm 0.6$ vs. $2.1 \pm 0.5 \mathrm{mmHgs} / \mathrm{cm}, \mathrm{p}<0.05)$. No significant differences in $\mathrm{dCA}$ gain and phase were found. In terms of the WKM approach, in the order $\mathrm{C} \rightarrow \mathrm{MCI} \rightarrow \mathrm{AD}$ groups showed about equal arterial resistance and peripheral compliance, but increased peripheral vasculature resistance ( $26 \pm 2$ vs. $36 \pm 3$ $\mathrm{mmHgs} / \mathrm{ml}$ in $\mathrm{C}$ resp. $\mathrm{AD}, \mathrm{p}=0.004)$.

In conclusion, $\mathrm{AD}$ patients compared to $\mathrm{MCI}$ patients and controls have increased CVRi, whereas dCA parameters do not seem to differentiate $A D$ patients. For MCI patients CVRi might have predictive value in developing AD. 


\section{Introduction}

Dementia is one of the principal neurological disorders in elderly. Recent studies $[11,15,21]$ have indicated that vascular risk factors are involved in the pathogenesis of cognitive disorders and dementia. For adequate function, the brain is critically dependent on continuous blood supply. Therefore, the cerebral vasculature is endowed with neurovascular control mechanisms that assure that the blood supply of the brain is commensurate to the energy needs of its cellular constituents. The regulation of cerebral blood flow (CBF) during brain activity involves the coordinated interaction of neurons, glia, and vascular cells. Alterations of the vascular regulatory mechanisms may lead to brain dysfunction and disease. A more and more emerging view is that cerebrovascular dysregulation is a feature not only of cerebrovascular pathologies, such as stroke, but also of neurodegenerative conditions, such as Alzheimer's disease (AD) [11]. First epidemiological studies have shown that risk factors for vascular diseases are important risk factors for AD [5]. Small ischemic lesions substantially aggravate the dementia [26]. Interaction of cerebral ischemia with AD pathology enhances the clinical manifestations of the disease. Moreover, AD patients have more severe atherosclerosis in large cerebral arteries at the base of the brain (circle of Willis) than age-matched controls without $\mathrm{AD}[19]$.

$\mathrm{AD}$ is the most common form of dementia and is often characterized by deposition of amyloid $\beta$-peptide in the blood vessels (amyloid angiopathy). Cerebral micro vessels are reduced in number and cerebrovascular function is alsoaltered in $\mathrm{AD}$. Resting $\mathrm{CBF}$ is reduced and the increase in CBF produced by activation (neurovascular coupling) is attenuated [25]. The cerebrovascular dysfunction often precedes the onset of cognitive impairment suggesting a role in the mechanisms of dementia [11]. Iadecola et al [11] pose a hypothetical time-course of the interplay between vascular dysregulation, neuropathological alterations and decline in brain function in AD. In the latent phase vascular dysregulation is already apparent when patients are asymptomatic. During the prodromal phase neuropathological alterations begin to manifest and cognitive function begins to decline. At this time, cognitive alterations are likely to result from amyloid $B$ peptide $(A ß)$ induced neuronal dysfunction and vascular dysregulation. As the disease progresses, the neuropathological changes evolve. Cerebrovascular disease deteriorates in parallel with cognitive function, reflecting in addition to $A ß$ induced vascular effects, the deleterious cerebrovascular effects of synaptic loss and vascular amyloid. In the late phase of the disease, brain function and vascular regulation are maximally compromised. 


\section{Chapter 4}

Cerebral autoregulation (CA) is one of the fundamental properties of the cerebral circulation through which $\mathrm{CBF}$ is maintained relatively constant despite variations in mean arterial pressure (MAP) within a certain range. To maintain flow in the autoregulated range of MAP, cerebral resistance vessels undergo vasoconstriction during hypertension and vasodilatation during hypotension. Therefore, failure of vasoconstriction and/or vasodilatation may result in CA disruption rendering the brain more susceptible to fluctuations in MAP.

Recently, results were published from a study describing specific abnormalities in $\mathrm{AD}$ in particular increased vessel flow resistance [4]. Cerebral blood flow was severely compromised during dynamic squat-stand procedures, and this was interpreted as reduced capability to counteract blood pressure changes in patients with early AD. To our knowledge, dynamic cerebral autoregulation (dCA) has not been studied in patients with mild cognitive impairment (MCI) and compared to $\mathrm{AD}$ and controls (C). MCI describes the transitional phase between normal aging and dementia. Patients with MCI are at increased risk for developing AD. Maybe impaired cerebral autoregulation can be a marker of developing $\mathrm{AD}$ in patients with MCI. Because vascular dysfunction might be a precursor of developing dementia it seems worthwhile to investigate parameters of vascular function in these groups.

A common approach to quantify the dynamics of cerebral autoregulation $[3,16$, 32] is transfer function analysis (TFA) of the presumed linear control system with arterial blood pressure (ABP) as the input and cerebral blood flow velocity (CBFV) as the output signal. Data analysis methods such as TFA are descriptive techniques quantifying the relation between $\mathrm{ABP}$ and $\mathrm{CBFV}$ without any assumption of the underlying physiology. To gain more insight in the results of this analysis with respect to physiological parameters a windkessel model (WKM) proposed by Zhang et al [31] can be fitted. In this way three parameters representing arterial vessel resistance $\left(R_{\mathrm{a}}\right)$, peripheral vessel resistance $\left(\mathrm{R}_{\mathrm{p}}\right)$ and vessel compliance $\left(\mathrm{C}_{\mathrm{p}}\right)$ can be estimated.

The objective of this study was to investigate TFA quantified cerebral autoregulation using spontaneous blood pressure variations in subjects with $\mathrm{AD}$ type dementia, $\mathrm{MCI}$ /prodromal $\mathrm{AD}$ and healthy controls. We hypothesized that $\mathrm{dCA}$ is impaired in $\mathrm{AD}$ patients compared to healthy controls. Additionally, we hypothesized that in MCI, as a possible prodromal phase of $\mathrm{AD}$, cerebral autoregulation shows reduced capacity. The model approach possibly can be used to identify specific physiological changes. 


\section{Patients and methods}

In this study $17 \mathrm{AD}$ patients, $19 \mathrm{MCI}$ patients and 20 matched healthy controls subjects $(\mathrm{C})$ were investigated. The groups were matched for age, sex and level of education. Patients with AD and MCI were selected from the Memory Clinic of the Maastricht University Medical Centre (MUMC). Control subjects were recruited from an existing cohort of elderly and through colleagues and personal acquaintances. The Institutional Review Board of the MUMC approved the study and all subjects gave written informed consent.

$\mathrm{AD}$ patients were diagnosed according to the DSM-IV criteria for dementia [1], and NINCDS-ADRDA criteria [13]. Nineteen MCI patients were included. Sixteen of these subjects (84\%) fulfilled the MCI criteria of Petersen et al [17], defined as a subjective cognitive complaint, objectified by an impaired cognitive performance; no significant impairments in daily living and no dementia. According to this definition, 11 subjects (69\%) had amnestic MCI, operationalized as a score below -1.5 standard deviation on the memory domain), and 5 subjects (31\%) had non-amnestic MCI, defined as a score below - 1.5 standard deviation on the nonmemory domain (more details are described below). Three subjects were clinically representative for the MCI syndrome, but did not reach the threshold of -1.5 $\mathrm{SD}$ on the cognitive tests. Exclusion criteria were any somatic, psychiatric or neurological disorder that may have caused the cognitive impairment. In addition, patients were excluded if diagnosed with Vascular Dementia, according to NINDS/AIREN criteria [20]. Also patients with diabetes and/or cardiovascular disease were excluded. Subjects with a stenosis of the common or internal carotid artery or the middle cerebral artery (MCA) of more than $50 \%$, as detected by extracranial and/or transcranial colour-coded duplex, were excluded as were subjects without a temporal bone window.

The electrocardiogram (ECG) and non-invasive ABP were measured using a Task Force Monitor (CN Systems ${ }^{\circledR}$, Austria). A transcranial Doppler system (Multidop X4, DWL ${ }^{\circledR}$, Sipplingen, Germany) was used to measure CBFV in the main stem of both the right and left MCA. Two $2 \mathrm{MHz}$ probes were held in position by a special frame. Patients were in supine position with their eyes open during all 15-minute recordings and were breathing spontaneously. Based on analysis of available MRI images of AD $(n=17)$ and MCI $(n=15)$ patients scores were obtained for medial temporal lobe atrophy (MTA)[22], general cortical atrophy (GCA), age-related white matter changes (ARWMC) and Fazekas score [7]. All subjects underwent a neuropsychological assessment. The measured cognitive domains included general cognitive functioning (Mini Mental State Examination, MMSE) [8], memory (immediate and delayed recall of the Auditory Verbal Learning Test [30] or the story of the Rivermead Behavioral Memory Test 
[27], speed of information processing (Trail Making Test, part A [18]; Stroop, card I and II [28]; and Symbol Digit Modality Test [24]), executive functioning (Trail Making Test, part B: Stroop, card III), and verbal fluency (fluency animals, 60 seconds) [29].

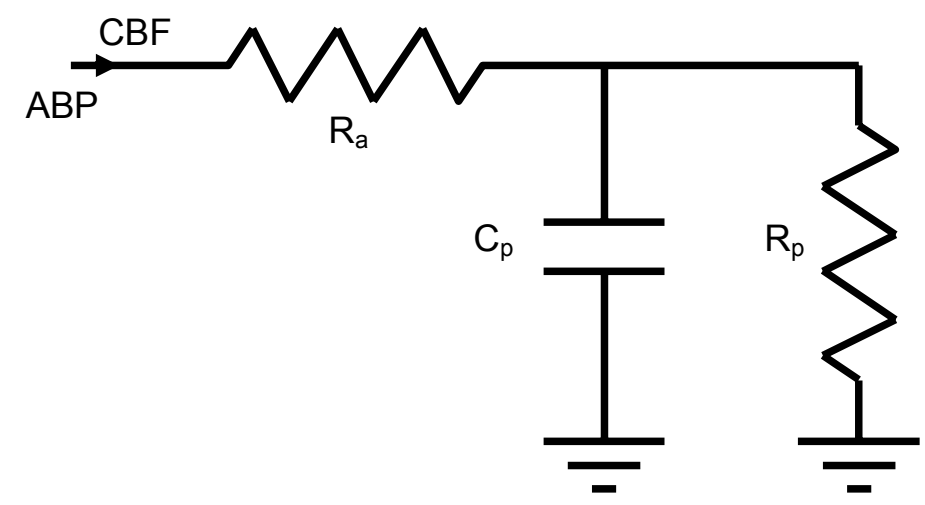

Figure 1 Windkessel model consisting of arterial resistance $R_{a}$, peripheral resistance

$\mathrm{R}_{\mathrm{p}}$ and peripheral compliance $\mathrm{C}_{\mathrm{p}}$ with pressure $\mathrm{ABP}$ representing arterial blood pressure and CBF cerebral blood flow.

\section{Data analysis}

Raw data from the Task Force Monitor were exported to an in-house made acquisition and analysis software package (AFOnew version 2.48, IDEE Maastricht ${ }^{\circledR}$, the Netherlands). An automatic algorithm detected the ECG R-waves and determined the subsequent diastolic, mean and systolic blood pressure and corresponding end diastolic, mean and peak systolic CBFV. Artefacts were removed manually by linear interpolation. The beat-to-beat values of $A B P$ and CBFV were exported to Matlab and resampled at $5 \mathrm{~Hz}$ using spline interpolation [16]. ABP and CBFV were normalized with respect to the mean. This resulted in zero-mean signals suited for spectral analysis to estimate the transfer function. Estimation of the transfer function was performed as previously described [10]. Phase and gain were evaluated for different frequency bands of autoregulation namely Total $(0.04-0.11 \mathrm{~Hz})$, very low frequency VLF $(0.04-0.06 \mathrm{~Hz}), 01(0.1 \mathrm{~Hz})$, low frequency LF (0.06-0.11 Hz). In order to compare the results (addition JC in abbreviations) reported by Claassen et al [4] with ours, we also computed the different CA parameters for the frequency bands VLFJC (0.02-0.07 Hz), LFJC $(0.07-0.2 \mathrm{~Hz})$ and HFJC $(0.2-0.35 \mathrm{~Hz})$. To be strict in acceptance of data, the coherence threshold applied is three times the previously described theoretically derived value [9]. With 15 minute recordings this means only recordings with a 
coherence of at least 0.18 were included. Recordings were excluded from further analysis, if nowhere in the frequency range below $0.25 \mathrm{~Hz}$ a coherence of 0.18 was reached. From fitting the first 5 seconds of the step response function the autoregulation index (ARI) was determined in the same way as described previously [9].

\section{Model description of dCA data}

The layout of the WKM is shown in figure 1. It consists of two resistors and one capacitor. Resistor $\mathrm{R}_{\mathrm{a}}$ represents flow resistance for arterioles distal to the MCA. $\mathrm{R}_{\mathrm{p}}$ represents more peripheral resistance in the microcirculation. $C_{p}$ represents compliance of the microcirculation. The transfer function from pressure $\mathrm{ABP}$ to flow $\mathrm{CBF}$ can be described in the Laplace domain with equation 1:

$$
H_{W K M}=\frac{C B F}{A B P}=\frac{C_{p} R_{p} s+1}{C_{p} R_{p} R_{a} s+R_{p}+R_{a}}
$$

where $H_{W K M}$ is the transfer function between arterial blood pressure $(A B P)$ and cerebral blood flow $(C B F) . C_{p}$ is peripheral compliance, $R_{p}$ is peripheral resistance, $R_{a}$ is arterial resistance and $s$ is the Laplace transform variable. Although the model parameters $C_{p}, R_{p}$ and $R_{a}$ are time-invariant system parameters, the transfer function of the model in equation 1 describes behaviour in response to blood pressure changes that can be related to the estimated transfer function of dCA calculated through cross spectral analysis.

To fit the WKM and convert the measured flow velocity into a flow for the model (CBFV $\rightarrow \mathrm{CBF}$ ) CBFV has to be multiplied by a cross sectional area A. Therefore, an internal MCA diameter of $3 \mathrm{~mm}$ was assumed [14], resulting in A of $\pi(1.5)^{2}=0.0707 \mathrm{~cm}^{2}$. This assumption obviously introduces noise in the results since not everyone will have a MCA caliber of $3 \mathrm{~mm}$. To estimate WKM parameters $R_{a}, R_{p}$ and $C_{p}$, an error function is minimized iteratively using the "fsolve" function in Matlab with a Levenberg-Marquardt algorithm.

$$
\begin{aligned}
& \varepsilon_{k}=\left|H_{T F A}\left(f_{i}\right)\right|-\left|H_{W K M}\left(f_{i}\right)\right|, k=1 . .8, i=4 . .11 \\
& \varepsilon_{k}=\operatorname{angle}\left\{H_{T F A}\left(f_{i}\right)\right\}-\operatorname{angle}\left\{H_{W K M}\left(f_{i}\right)\right\}, k=9 . .16, i=4 . .11 \\
& \varepsilon_{k}=A \bullet\left(R_{a}+R_{p}\right)-C V R i, k=17
\end{aligned}
$$

The error function consists of the set of expressions in equation 2 that represent the differences of: 1) TFA and WKM gain in the frequency band from $0.06-0.2 \mathrm{~Hz}$ 2) TFA and WKM phase in the frequency band from $0.06-0.2 \mathrm{~Hz}$ and 3) CVRi and $\left(\mathrm{R}_{\mathrm{a}}+\mathrm{R}_{\mathrm{p}}\right) * A$. Consequently, for both gain and phase eight data points (in the frequency range $0.06-0.20 \mathrm{~Hz}$ ) from the calculated transfer function are compared with corresponding frequency WKM values. For CVRi it consists of a 
single value difference. The fit error was calculated as the sum of squares of the error function components as expressed in equation 3.

$\varepsilon_{\text {fit }}=\sum_{k=1}^{17} \varepsilon_{k}^{2}$

Table 1 General characteristics of subjects with AD, MCI and controls.

MMSE $=$ mini mental state evaluation, $\mathrm{MTA}=$ medial temporal lobe atrophy, $\mathrm{sBP}$, $\mathrm{dBP}, \mathrm{mBP}=$ systolic, diastolic, mean blood pressure, $\mathrm{mCBFV}=$ mean cerebral blood flow velocity, CVRi = cerebrovascular resistivity index

\begin{tabular}{|c|c|c|c|c|}
\hline & $\mathrm{AD}(\mathrm{n}=15)$ & MCI (n=19) & $C(n=20)$ & \\
\hline Male/female & $8 / 7$ & $11 / 8$ & $10 / 10$ & NS \\
\hline Age [year] & $\begin{array}{c}72(2) \\
{[58-87]}\end{array}$ & $\begin{array}{c}70(2) \\
{[58-80]}\end{array}$ & $\begin{array}{c}70(1) \\
{[59-78]}\end{array}$ & NS \\
\hline \multicolumn{5}{|l|}{$\begin{array}{l}\text { Level of } \\
\text { Education [\%] }\end{array}$} \\
\hline Lower & 53 & 37 & 45 & NS \\
\hline Middle & 33 & 16 & 35 & NS \\
\hline Higher & 13 & 47 & 20 & NS \\
\hline MMSE & $\begin{array}{c}19.8(1.2) \\
{[12-27]}\end{array}$ & $\begin{array}{c}27.6(0.3) \\
{[24-30]}\end{array}$ & $\begin{array}{c}29.0(0.3) \\
{[26-30]}\end{array}$ & $\begin{array}{l}\text { AD-MCI } \\
\mathrm{p}<0.001 \\
\text { AD-C } p<0.001\end{array}$ \\
\hline MTA > 2 [\%] & 80 & 33 & no data & $\mathrm{p}=0.011^{*}$ \\
\hline $\begin{array}{l}\text { End-tidal } \mathrm{CO}_{2} \\
{[\%]}\end{array}$ & $6.0(0.4)$ & $6.3(0.2)$ & $5.3(0.1)$ & $\begin{array}{l}\text { MCI-C } \\
p=0.006\end{array}$ \\
\hline $\mathrm{sBP}[\mathrm{mmHg}]$ & $\begin{array}{c}134(4) \\
{[116-156]}\end{array}$ & $\begin{array}{c}133(2) \\
{[116-150]}\end{array}$ & $\begin{array}{c}133(3) \\
{[107-158]}\end{array}$ & NS \\
\hline $\mathrm{dBP}[\mathrm{mmHg}]$ & $\begin{array}{c}89(2) \\
{[78-101]}\end{array}$ & $\begin{array}{c}86(2) \\
{[70-105]}\end{array}$ & $\begin{array}{c}88(2) \\
{[61-104]}\end{array}$ & NS \\
\hline $\mathrm{mBP}[\mathrm{mmHg}]$ & $\begin{array}{l}104(2) \\
{[90-119]}\end{array}$ & $\begin{array}{c}101(2) \\
{[88-117]}\end{array}$ & $\begin{array}{l}103(3) \\
{[76-119]}\end{array}$ & NS \\
\hline $\mathrm{mCBFV}$ & $37.3(2.3)$ & $46.6(2.8)$ & $51.0(2.8)$ & AD-C \\
\hline$[\mathrm{cm} / \mathrm{s}]$ & [26-54] & {$[27-77]$} & {$[32-82]$} & $\mathrm{p}=0.003$ \\
\hline $\begin{array}{l}\text { CVRi } \\
{[\mathrm{mmHgs} / \mathrm{cm}]}\end{array}$ & $2.9(0.2)$ & $2.3(0.1)$ & $2.1(0.1)$ & $\begin{array}{l}A D-C \\
p=0.002 \\
A D-M C I \\
p=0.02\end{array}$ \\
\hline
\end{tabular}

All data are presented as mean (SEM) [ min max].

$\mathrm{n}=$ number of subjects, $\mathrm{p}$-values of multifactor Anova with Sidak correction

* p-value of Mann-Whitney test 


\section{Statistical analysis}

Statistical analyses were performed using the Statistical Package for Social Sciences (SPSS) version 16.0 for Windows. Between group data were statistically compared using multifactor ANOVA (Sidak correction). Confounders were only included if significant correlation existed with the parameter studied. In this case multifactor ANOVA was repeated with correction for the confounders. If during the course of the study MCI patients convert into Alzheimer's disease according to the diagnosis criteria, the results for these converters will be analysed separately.

\section{Results}

In two $\mathrm{AD}$ cases coherence was below the threshold of 0.18 at all frequencies up to $0.25 \mathrm{~Hz}$ and these patients were excluded from the results, thus leaving $15 \mathrm{AD}$ patients. General subject characteristics are shown in table 1. Age and sex matching between the groups was adequate since there were no significant differences. The MMSE was significantly lower in $\mathrm{AD}$ compared to both MCI and C. Blood pressure values (systolic sBP, diastolic $\mathrm{dBP}$ and mean $\mathrm{mBP}$ ) did not differ between the groups. Mean cerebral blood flow velocity (mCBFV) data from right and left MCA were pooled as there were no significant right-left differences. In $\mathrm{AD} \mathrm{mCBFV}$ was significantly lower compared to $\mathrm{C}$ and tended to be lower compared to $\mathrm{MCI}(\mathrm{p}=0.068)$. CVRi was significantly higher in $\mathrm{AD}$ compared to both $\mathrm{MCI}$ and $\mathrm{C}$.

Table $2 \mathrm{dCA}$ parameters of subjects with AD, MCI and controls.

\begin{tabular}{llll}
\multicolumn{4}{c}{ ARI $=$ autoregulatory index } \\
\hline AD $(\mathrm{n}=15)$ & MCI $(\mathrm{n}=19)$ & $\mathrm{C}(\mathrm{n}=20)$ \\
\hline CoherenceVLF & $0.25(0.03)$ & $0.27(0.03)$ & $0.28(0.03)$ \\
CoherenceLF & $0.47(0.07)$ & $0.53(0.04)$ & $0.53(0.03)$ \\
CoherenceTotal & $0.38(0.05)$ & $0.42(0.03)$ & $0.43(0.03)$ \\
GainVLF [\%/\%] & $0.85(0.07)$ & $0.96(0.08)$ & $1.03(0.10)$ \\
GainLF [\%/\%] & $1.32(0.12)$ & $1.51(0.09)$ & $1.58(0.11)$ \\
GainTotal [\%/\%] & $1.13(0.08)$ & $1.29(0.08)$ & $1.36(0.09)$ \\
PhaseVLF [rad] & $0.85(0.13)$ & $0.66(0.06)$ & $0.78(0.11)$ \\
PhaseLF [rad] & $1.00(0.09)$ & $0.94(0.06)$ & $0.84(0.05)$ \\
PhaseTotal [rad] & $0.94(0.09)$ & $0.83(0.05)$ & $0.81(0.06)$ \\
ARI & $6.1(0.2)$ & $5.8(0.2)$ & $5.5(0.2)$ \\
\hline
\end{tabular}

All data are presented as mean (SEM).

$\mathrm{n}=$ number of subjects, no significant differences with multifactor Anova with Sidak correction 


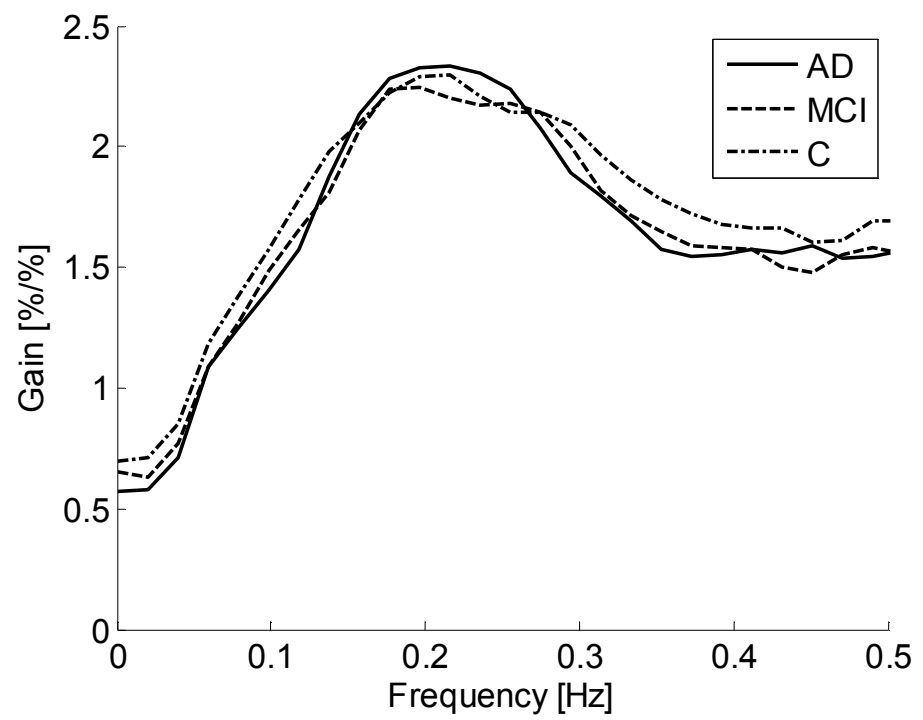

Figure 2 Group average gain spectrum for AD (solid), MCI (dashed) and C (dashdotted).

\section{$d C A$ linear transfer function results}

Table 2 shows a summary of dCA parameters. Figure 2 shows the group averaged gain and figure 3 the group averaged phase plots. None of the dCA parameters showed significant differences between the groups. Also all other parameters including the dCA parameters in the specific frequency bands used by Claassen $e t$ al [4], did not reveal significant group differences.

Table 3 Windkessel model parameters of subjects with AD, MCI and controls

\begin{tabular}{lcccc}
\hline & AD $(\mathrm{n}=15)$ & $\mathrm{MCI}(\mathrm{n}=19)$ & $\mathrm{C}(\mathrm{n}=20)$ & \\
\hline $\mathrm{R}_{\mathrm{a}}$ & $5.6(0.7)$ & $4.9(0.4)$ & $5.2(0.5)$ & $\mathrm{NS}$ \\
{$[\mathrm{mmHgs} / \mathrm{ml}]$} & {$[2-10]$} & {$[2-9]$} & {$[0-11]$} & \\
$\mathrm{R}_{\mathrm{p}}$ & $36(3)$ & $29(2)$ & $26(2)$ & $\mathrm{AD}-\mathrm{C}$ \\
{$[\mathrm{mmHgs} / \mathrm{ml}]$} & {$[21-59]$} & {$[16-52]$} & {$[14-48]$} & $\mathrm{p}=0.004$ \\
$\mathrm{C}_{\mathrm{p}}$ & $0.24(0.02)$ & $0.25(0.02)$ & $0.29(0.04)$ & $\mathrm{NS}$ \\
{$[\mathrm{ml} / \mathrm{mmHg}]$} & {$[.12-.44]$} & {$[.13-.47]$} & {$[.13-.98]$} & \\
Fit error $\varepsilon_{\text {fit }}$ & $2.1(0.5)$ & $1.3(0.3)$ & $1.7(0.5)$ & $\mathrm{NS}$ \\
& {$[0.4-7]$} & {$[0.04-5]$} & {$[.3-10]$} & \\
\hline
\end{tabular}

All data are presented as mean (SEM) [min max].

$\mathrm{n}=$ number of subjects, $\mathrm{p}$-values of multifactor Anova with Sidak correction 


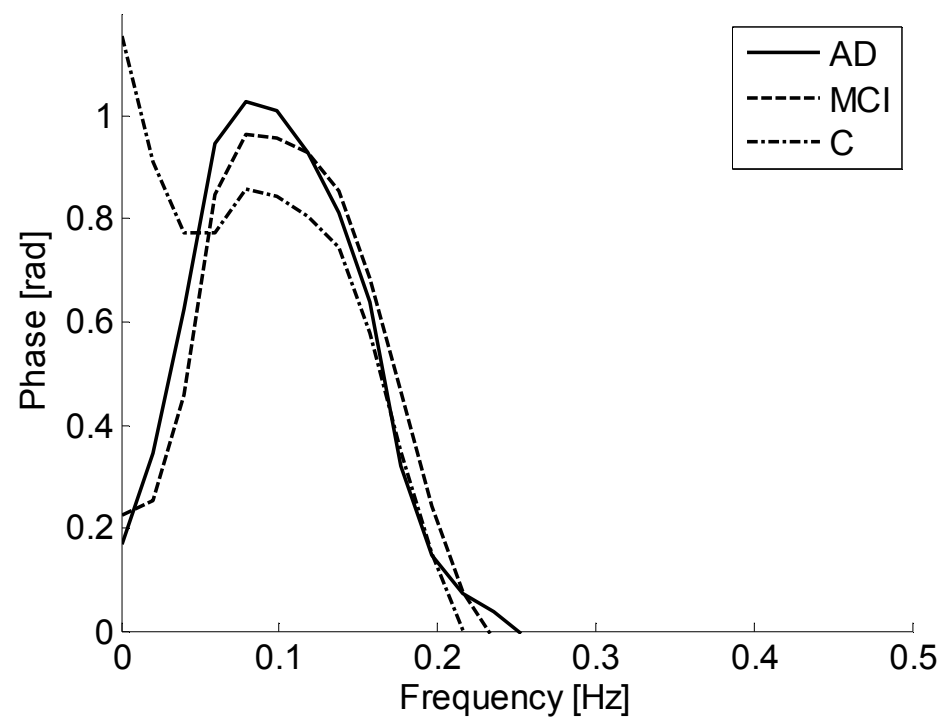

Figure 3 Group average phase spectrum for AD (solid), MCI (dashed) and C (dashdotted).

\section{Model description}

Estimation of the WKM parameters was successful in all recordings. Table 3 summarizes the three model parameters for the three groups. Peripheral resistance $\mathrm{R}_{\mathrm{p}}$ was significantly increased in $\mathrm{AD}$ compared to $\mathrm{C}$. MCI was in between $\mathrm{AD}$ and $\mathrm{C}$ without significant differences to either of them. Entering the WKM parameters from table 3 in equation 1, gain and phase can be calculated respectively as the modulus and argument of the transfer function H. Plots of gain and phase versus frequency are shown in figure 4 and 5 showing no significant difference between groups. 
Chapter 4

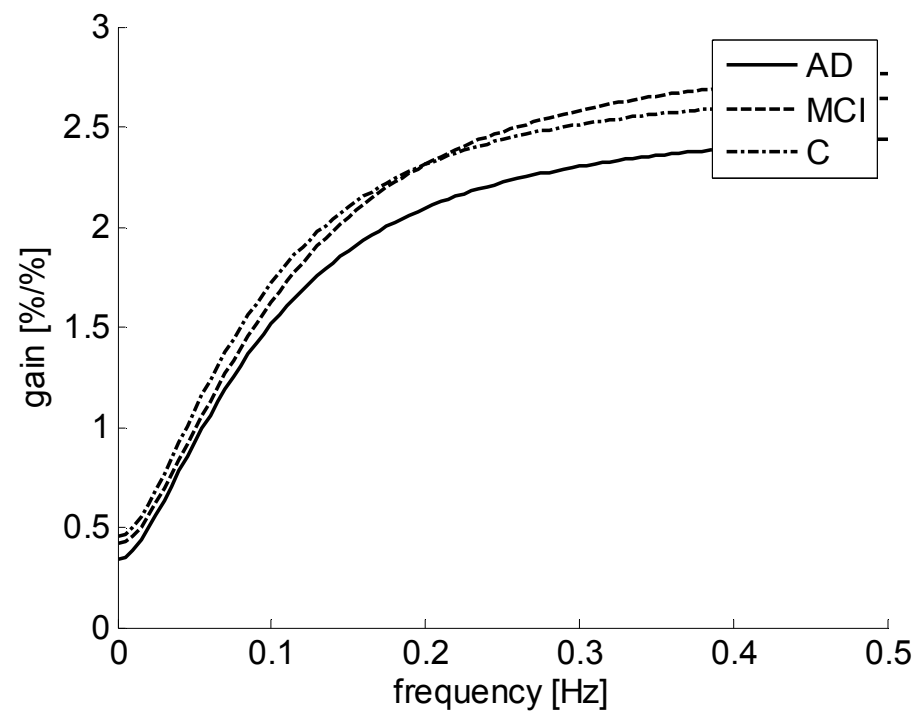

Figure 4 Gain plots for WKM group averaged parameters for AD (solid), MCI (dashed) and C (dash-dotted).

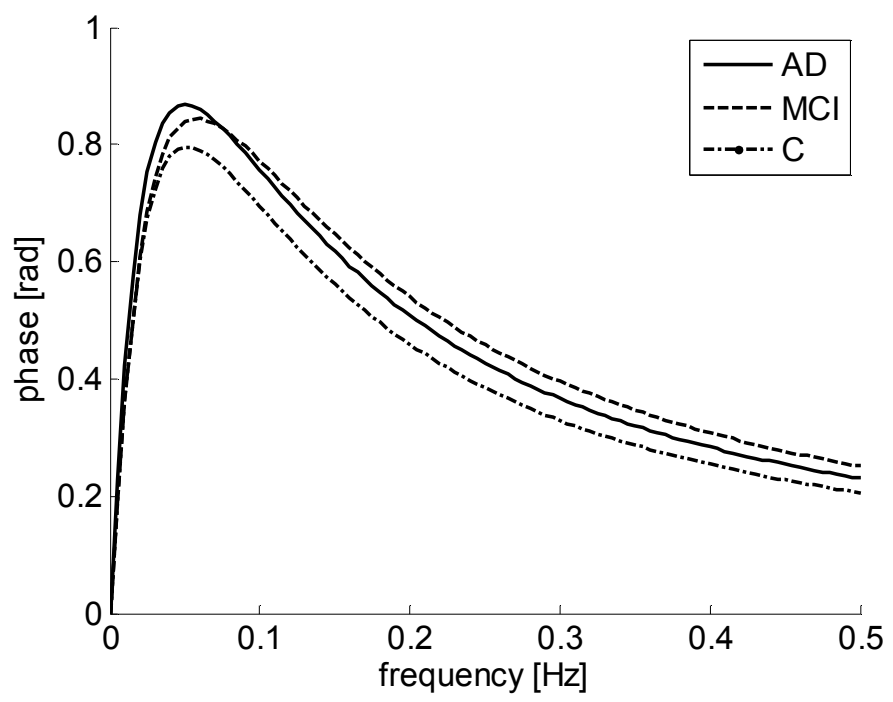

Figure 5 Phase plot for WKM group averaged parameters for AD (solid), MCI (dashed) and C (dash-dotted). 
Table 4 Significant Pearson (for sBP, $\mathrm{dBP}$ and $\mathrm{mBP}=$ systolic, diastolic and mean blood pressure) and Spearman (for GCA = general cortical atrophy, MTA = medial temporal lobe atrophy and MTA right) correlation coefficients between autoregulation parameters and general subject characteristics

\begin{tabular}{lcccccc}
\hline & $\mathrm{sBP}$ & $\mathrm{dBP}$ & $\mathrm{mBP}$ & $\mathrm{GCA}$ & $\mathrm{MTA}$ & $\begin{array}{c}\text { MTA } \\
\text { right }\end{array}$ \\
& $\mathrm{n}=54$ & $\mathrm{n}=54$ & $\mathrm{n}=54$ & $\mathrm{n}=30$ & $\mathrm{n}=30$ & $\mathrm{n}=30$ \\
\hline GainLF [\%/\%] & & & & & & \\
GainTotal [\%/\%] & & & & & & \\
PhaseVLF [rad] & $0.28^{*}$ & & & & & \\
PhaseLF [rad] & $0.37^{* *}$ & $0.31^{*}$ & $0.35^{* *}$ & & & \\
PhaseTotal [rad] & $0.41^{* *}$ & $0.29^{*}$ & $0.36^{* *}$ & & & \\
ARI & $0.29^{*}$ & & $0.28^{*}$ & & & \\
CVRi $[\mathrm{mmHgs} / \mathrm{cm}]$ & $0.28^{*}$ & $0.36^{* *}$ & & $0.38^{*}$ & $0.43^{*}$ & $0.49^{* *}$ \\
$\operatorname{Ra}[\mathrm{mmHgs} / \mathrm{ml}]$ & & & & $0.41^{*}$ & $0.46^{*}$ & $0.49^{* *}$ \\
$\mathrm{Rp}[\mathrm{mmHgs} / \mathrm{ml}]$ & $0.30^{*}$ & $0.37^{* *}$ & $0.36^{* *}$ & & & \\
$\mathrm{Cp}[\mathrm{ml} / \mathrm{mmHg}]$ & & & & & $0.38^{*}$ \\
\hline
\end{tabular}

\section{Correlations}

Significant correlations between autoregulation parameters and subject characteristics are shown in table 4 . To see if covariates exist for autoregulation parameters and to investigate dependencies between parameters bivariate correlations were calculated between general subject characteristics and autoregulation parameters. For continuous parameters (e.g .blood pressures) Pearson's correlation coefficient was calculated and for MRI parameters with only a few levels Spearman's correlation coefficient was calculated. In 4 MCI cases no MRI data was present. Comparing groups incorporating significant covariates did not show additional significant differences between groups nor did it abolish preexisting significant differences.

\section{Conversion of $\mathrm{MCl}$ into $A D$}

Since the beginning of the study five of the MCI patients have converted into AD. Post hoc we analysed if certain parameters could predict this conversion. We tested all parameters using a Mann-Whitney test for the converted $(n=5)$ and non-converted ( $\mathrm{n}=14) \mathrm{MCI}$ patients. Only mCBFV ( $35 \pm 5 \mathrm{vs} .51 \pm 11 \mathrm{~cm} / \mathrm{s}, \mathrm{p}<0.01)$ and CVRi were significantly different $(2.8 \pm 0.6$ vs. mean $2.1 \pm 0.5 \mathrm{mmHgs} / \mathrm{cm}$, $\mathrm{p}<0.05)$ for converted versus non-converted MCI patients. 


\section{Discussion}

In this study we found reduced mCBFV and correspondingly elevated CVRi in $\mathrm{AD}$ patients compared to matched controls. Also compared to MCI CVRi is significantly elevated in $\mathrm{AD}$. It has been argued that reduction in $\mathrm{CBF}$ in $\mathrm{AD}$ could be explained by loss of brain tissue due to the neurodegenerative processes, or by a reduction in brain metabolic demand that parallels cognitive decline [23]. We found increased medial temporal lobe atrophy in AD compared to $\mathrm{MCI}$, which could support the relation between loss of brain tissue and reduced cerebral blood flow. Also, recent fMRI data in $\mathrm{MCI}$ and $\mathrm{AD}$ are beginning to reveal relationships between abnormalities of functional activity in the medial temporal lobe memory system and in functionally connected brain regions [6]. Since inclusion of $\mathrm{AD}$ and $\mathrm{MCI}$ subjects in our study was from an existing cohort of the Memory Clinic in our hospital, MRI data was sometimes acquired at an earlier stage compared to the autoregulation tests. Only in nine subjects autoregulation tests were performed within one year from the MRI acquisition date. While disease progresses this could have blurred the correlation between atrophy and autoregulation data.

From the group of MCI patients, during the course of this study, five converted to $\mathrm{AD}$ on average two years with a standard deviation of one year after the cerebrovascular measurements were performed. The results for this subgroup compared to the non-converted MCI patients showed mCBFV to be significantly lower and CVRi to be significantly higher. Although the number of converters is small this strongly suggests the value of these parameters in the diagnosis of AD. It could therefore be worthwhile to evaluate the value of screening elderly and specifically MCI patients for these parameters.

No significant differences were found between the groups for dCA. This is in correspondence with findings of Claassen et al [4] for spontaneous fluctuations of blood pressure. However, they did show differences in gain during squat-stand maneuvers. They also showed increased spectral power in blood pressure and CBFV during these maneuvers. It could therefore be necessary to induce stronger challenges to the control of cerebral blood flow for changes in cerebral autoregulation to become apparent. By inducing stronger challenges such as squat-stand maneuvers maybe also differences between $\mathrm{MCI}$ and $\mathrm{C}$ might appear. New extensive and long term prospective research is needed to investigate this theory and to study the development from $\mathrm{MCI}$ into $\mathrm{AD}$ in relation to $\mathrm{dCA}$ parameters. Although we included roughly twice the amount of subjects as Claassen et al [4] did, the study might be underpowered to show differences in autoregulation 
parameters with spontaneous blood pressure variations in supine rest. Using the results from this study the group size needed to show significant differences between $\mathrm{AD}$ and $\mathrm{C}$ could be estimated. A sample size analysis for GainLF shows that two to three times as many subjects are needed to show possible significant differences $(\mathrm{p}<0.05)$ between $\mathrm{AD}$ and $\mathrm{C}$ with $80 \%$ power.

The windkessel model parameters that were fitted with the transfer function results show that the differences between $\mathrm{AD}, \mathrm{MCI}$ and $\mathrm{C}$ groups are specific for the peripheral vasculature, since the arterial resistance $R_{a}$ did not differ between the groups. The peripheral resistance $\mathrm{R}_{\mathrm{p}}$ is increased in $\mathrm{AD}$ compared to controls. This is in accordance with the increased CVRi we found. Bateman et al [2] also found increased resistance in AD using MRI to quantify cerebral haemodynamics. They also found compliance to be significantly lower (about $20 \%$ ) in AD compared to controls. We found compliance in AD to be $17 \%$ lower than in controls, which was not significant. Our results therefore do not provide evidence for additional benefit of using the windkessel model over simply determining CVRi. CVRi is much easier to determine and could prove its value in the multidimensional diagnosis of AD. With a correlation between CVRi and $\mathrm{R}_{\mathrm{p}}$ of 0.97 the parameters show to be almost identical.

The autoregulation parameters phase and ARI have positive correlations with blood pressure parameters, whereas autoregulation gain does not correlate with blood pressure. Apart from autoregulation, vascular compliance also elicits flow phase lead with respect to pressure due to fundamental hemodynamic behaviour of elastic tubes. As Zhang et al showed, in the WKM for low values of $\mathrm{C}_{\mathrm{p}}$ a positive correlation with phase exists and for high values of $\mathrm{C}_{\mathrm{p}}$ it is negatively correlated with phase [31]. We found no correlation between $\mathrm{C}_{\mathrm{p}}$ and blood pressure and therefore the correlation between phase, ARI and blood pressure seems to be determined by direct influence of blood pressure. So, the higher blood pressure the faster autoregulation performs showing cerebral autoregulation seems to adjust adequately to higher levels of blood pressure. CVRi and $\mathrm{R}_{\mathrm{p}}$ also positively correlate with blood pressure. For CVRi this can be easily explained by the formula used to calculate it. $\mathrm{R}_{\mathrm{p}}$, which is the only windkessel model parameter that significantly differs between $\mathrm{AD}$ and $\mathrm{C}$, is strongly correlated with CVRi and therefore seems to reflect best the differences in CVRi among these groups also indicating that vascular pathology manifests predominantly in the smaller arterioles. CVRi and the windkessel parameters $\mathrm{R}_{a}$ and $\mathrm{C}_{\mathrm{p}}$ positively correlate specifically with right medial temporal lobe atrophy, whereas CVRi and $\mathrm{R}_{\mathrm{a}}$ also correlate with the MTA average, but less strong. This side specific correlation is difficult to explain, but could indicate lateralisation of pathological cognitive function. 


\section{Chapter 4}

Of course the autoregulation parameters evaluated in this study will never be a single diagnostic parameter identifying which MCI patient will develop Alzheimer's disease and which one doesn't, but these parameters could help in determining the influence of vascular components in the disease spectrum of cognitive disorders and together with other factors could disentangle the complex relationship between cerebral blood flow and cognition. A multidisciplinary approach is needed to study vascular cognitive impairment [12]. We also performed extensive duplex and neuropsychological investigations. These results will be published in successive papers.

\section{Limitations}

TCD measures only relative velocity instead of absolute flow due to the unknown vessel diameter and insonation angle. Therefore, the assumed diameter of $3 \mathrm{~mm}$ for the WKM parameters increases noise and could mask differences in e.g. compliance.

The small number of patients in this study could limit the power to show significant differences. For the autoregulation parameter GainLF gain a post hoc analysis showed that at least twice the number of subjects would be needed to show a significant difference for this parameter. Since the recruitment was from an existing cohort of both AD and MCI the MRI data were not always acquired within the same time frame as the autoregulation measurement were performed. In only 9 patients the MRI was acquired maximally one year before the autoregulation test. This means that the condition of the cerebrovascular system could have been worsened with respect to the MRI results.

Medication can influence the relation between disease groups and their cerebrovascular results. Therefore, use of psychopharmacological medication was an exclusion criterion. Medication use was asked and almost every AD patient used a cholinesterase inhibitor. Due to ethical reasons this could not be stopped for the present study. A few AD patients (12\%) and MCI patients (32\%) used statines, which could influence autoregulation performance.

\section{Conclusion}

This is the first study comparing cerebral autoregulation in three subject groups namely AD, MCI and matched controls in which distinct changes in cerebral hemodynamics in patients with $\mathrm{AD}$ were observed. Increased cerebrovascular resistance is found, possibly due to enhanced vasoconstriction in $\mathrm{AD}$. CVRi values for MCI showed to be in between $\mathrm{AD}$ and controls but not significantly different from controls. In MCI decreased $\mathrm{mCBFV}$ and increased CVRi might be 
prognostic factors for developing AD. CVRi shows to be an easy to determine parameter and could be of value in the multidimensional diagnosis of AD.

Linear transfer analysis of cerebral autoregulation may be used as a tool to characterise the cerebrovascular dynamics in AD and particularly in MCI patients, but probably the control of cerebral blood flow needs to be more strongly provoked by inducing greater blood pressure variability. Regarding the latter, future studies should evaluate the predictive power of such measurements with regard to the development of MCI into $\mathrm{AD}$.

\section{Acknowledgements}

Research supported by: Internationale Stichting Alzheimer Onderzoek (ISAO) grant \# 06518 to JR.

The authors thank Prof. R.B. Panerai (University of Leicester, UK) for his valuable contributions to the manuscript.

\section{References}

1. Association AP editor. Diagnostic and Statistical Manual of Mental Disorders. Washington, DC: American Psychiatric Association, 1994.

2. Bateman GA, Levi CR, Schofield P, Wang Y, and Lovett EC. Quantitative measurement of cerebral haemodynamics in early vascular dementia and Alzheimer's disease. J Clin Neurosc 13: 563-568, 2006.

3. Blaber AP, Bondar RL, Stein F, Dunphy PT, Moradshahi P, Kassam MS, and Freeman R. Transfer function analysis of cerebral autoregulation dynamics in autonomic failure patients. Stroke 28: 1686-1692, 1997.

4. Claassen JA, Diaz-Arrastia R, Martin-Cook K, Levine BD, and Zhang R. Altered cerebral hemodynamics in early Alzheimer disease: a pilot study using transcranial Doppler. J Alzheimers Dis 17: 621-629, 2009.

5. de la Torre JC. Vascular Basis of Alzheimer's Pathogenesis. Ann N YAcad Sci 977: 196-215, 2002.

6. Dickerson BC, and Sperling RA. Functional abnormalities of the medial temporal lobe memory system in mild cognitive impairment and Alzheimer's disease: Insights from functional MRI studies. Neuropsychologia 46: 1624-1635, 2008.

7. Fazekas F, Chawluk JB, Alavi A, Hurtig HI, and Zimmerman RA. MR signal abnormalities at $1.5 \mathrm{~T}$ in Alzheimer's dementia and normal aging. AJR Am J Roentgenol 149: 351-356, 1987. 
8. Folstein MF, Folstein SE, and McHugh PR. "Mini-mental state". A practical method for grading the cognitive state of patients for the clinician. J Psychiatr Res 12: 189-198, 1975.

9. Gommer E, Shijaku E, Mess W, and Reulen J. Dynamic cerebral autoregulation: different signal processing methods without influence on results and reproducibility. Med Biol Eng Comput 1-8, 2010.

10. Gommer ED, Staals J, van Oostenbrugge RJ, Lodder J, Mess WH, and Reulen JP. Dynamic cerebral autoregulation and cerebrovascular reactivity: a comparative study in lacunar infarct patients. Physiol Meas 29: 1293-1303, 2008.

11. Iadecola C. Neurovascular regulation in the normal brain and in Alzheimer's disease. Nat Rev Neurosci 5: 347-360, 2004.

12. Marshall RS, and Lazar RM. Pumps, Aqueducts, and Drought Management: Vascular Physiology in Vascular Cognitive Impairment. Stroke 42: 221-226, 2011.

13. McKhann G, Drachman D, Folstein M, Katzman R, Price D, and Stadlan EM. Clinical diagnosis of Alzheimer's disease: report of the NINCDSADRDA Work Group under the auspices of Department of Health and Human Services Task Force on Alzheimer's Disease. Neurology 34: 939944, 1984.

14. Newell DW, and Aaslid R. Transcranial Doppler. New York: Raven Press, 1992.

15. Niwa K, Kazama K, Younkin L, Younkin SG, Carlson GA, and Iadecola C. Cerebrovascular autoregulation is profoundly impaired in mice overexpressing amyloid precursor protein. Am J Physiol Heart Circ Physiol 283: H315-323, 2002.

16. Panerai RB, Dawson SL, and Potter JF. Linear and nonlinear analysis of human dynamic cerebral autoregulation. Am J Physiol 277: H1089-1099, 1999.

17. Petersen RC, Smith GE, Waring SC, Ivnik RJ, Tangalos EG, and Kokmen E. Mild cognitive impairment: clinical characterization and outcome. Arch Neurol 56: 303-308, 1999.

18. Reitan RM. Validity of the Trial Making Test as an indication of organic brain damage. Percept Motor Skills 8: 271-276, 1958.

19. Roher AE, Esh C, Kokjohn TA, Kalback W, Luehrs DC, Seward JD, Sue LI, and Beach TG. Circle of Willis Atherosclerosis Is a Risk Factor for Sporadic Alzheimer's Disease. Arterioscler Thromb Vasc Biol 23: 20552062, 2003. 
20. Roman GC, Tatemichi TK, Erkinjuntti T, Cummings JL, Masdeu JC, Garcia JH, Amaducci L, Orgogozo JM, Brun A, Hofman A, and et al. Vascular dementia: diagnostic criteria for research studies. Report of the NINDS-AIREN International Workshop. Neurology 43: 250-260, 1993.

21. Rosengarten B, Paulsen S, Molnar S, Kaschel R, Gallhofer B, and Kaps M. Acetylcholine esterase inhibitor donepezil improves dynamic cerebrovascular regulation in Alzheimer patients. J Neurol 253:58-64, 2006.

22. Scheltens P, Leys D, Barkhof F, Huglo D, Weinstein HC, Vermersch P, Kuiper M, Steinling M, Wolters EC, and Valk J. Atrophy of medial temporal lobes on MRI in "probable" Alzheimer's disease and normal ageing: diagnostic value and neuropsychological correlates. J Neurol Neurosurg Psychiatry 55: 967-972, 1992.

23. Shih WJ, Ashford JW, Coupal JJ, Ryo YU, Stipp VV, Magoun SL, and Gross K. Consecutive brain SPECT surface three-dimensional displays show progression of cerebral cortical abnormalities in Alzheimer's disease. Clin Nucl Med 24: 773-777, 1999.

24. Smith A editor. Symbol Digit Modalities Test. Los Angeles, California: Western Psychological Services, 1973.

25. Smith EE, Vijayappa M, Lima F, Delgado P, Wendell L, Rosand J, and Greenberg SM. Impaired visual evoked flow velocity response in cerebral amyloid angiopathy. Neurology 71: 1424-1430, 2008.

26. Snowdon DA. Healthy aging and dementia: findings from the Nun Study. Ann Intern Med 139: 450-454, 2003.

27. van Balen HHG, and Wimmers MFHG editors. Rivermead Behavioural Memory Test: Normeringsgegevens voor Nederland en Vlaanderen. Lisse: Swets \& Zeitlinger, 1993.

28. Van der Elst W, Van Boxtel MPJ, Van Breukelen GJP, and Jolles J. The Stroop Color-Word Test. Assessment 13: 62-79, 2006.

29. Van Der Elst WIM, Van Boxtel MPJ, Van Breukelen GJP, and Jolles J. Normative data for the Animal, Profession and Letter M Naming verbal fluency tests for Dutch speaking participants and the effects of age, education, and sex. J Internat Neuropsychol Soc 12: 80-89, 2006.

30. Van Der Elst WIM, Van Boxtel MPJ, Van Breukelen GJP, and Jolles J. Rey's verbal learning test: Normative data for 1855 healthy participants aged 24-81 years and the influence of age, sex, education, and mode of presentation. J Internat Neuropsychol Soc 11: 290-302, 2005.

31. Zhang R, Behbehani K, and Levine BD. Dynamic pressure-flow relationship of the cerebral circulation during acute increase in arterial pressure. J Physiol 587: 2567-2577, 2009. 
Chapter 4

32. Zhang R, Zuckerman JH, Giller CA, and Levine BD. Transfer function analysis of dynamic cerebral autoregulation in humans. Am J Physiol 274: H233-241, 1998. 


\section{Chapter 5}

Visually evoked blood flow responses and interaction with dynamic cerebral autoregulation: correction for blood pressure variation

E D Gommer, J G Bogaarts, E G H J Martens, W H Mess, J P H Reulen. Visually evoked blood flow responses and interaction with dynamic cerebral autoregulation: correction for blood pressure variation.

Submitted for publication 


\section{Abstract}

Visually evoked flow responses (VEFR) recorded using transcranial Doppler ultrasonography are often quantified using a dynamic model of neurovascular coupling (NVC). The VEFR is seen as the model's response to a visual step input stimulus. However, the continuously active process of dynamic cerebral autoregulation (dCA) compensating cerebral blood flow for blood pressure fluctuations may induce changes of cerebral blood flow velocity (CBFV) as well. The effect of blood pressure variability on VEFR is evaluated by separately modeling the dCA induced effects of beat-to-beat measured blood pressure related CBFV changes.

VEFR NVC parameters of 71 subjects are estimated using two models: the RG model is a well-known second order dynamic NVC model proposed by Rosengarten et al [21]. The second $\mathrm{RG}_{\mathrm{CA}}$ model extends the $\mathrm{RG}$ model with a $\mathrm{CBFV}$ contributing component being the output of a dCA model driven by blood pressure as input.

Both models were evaluated for mean and systolic CBFV VEFR responses. The model-to-data fit errors from the $\mathrm{RG}_{\mathrm{CA}}$ mean and systolic blood pressure corrected model were significantly lower compared to the RG model: mean $0.8 \% \pm$ 0.6 vs. $2.4 \% \pm 2.8 \mathrm{p}<0.001$ systolic $1.5 \% \pm 1.2$ vs. $2.2 \% \pm 2.6 \mathrm{p}<0.001$. The confidence bounds of all $\mathrm{RG}_{\mathrm{CA}}$ estimated NVC model parameters were significantly $(\mathrm{p}<0.005)$ narrowed.

In conclusion, blood pressure correction of NVC responses by including cerebral autoregulation in model fitting of averaged VEFR responses results in significantly lower fit errors and by that in more reliable model parameter estimation. Blood pressure correction is more effective when mean instead of systolic CBFV responses are used. Measurement and quantification of NVC should include beat-to-beat blood pressure measurement. 


\section{Introduction}

Measurement of a visually evoked blood flow response in the posterior cerebral artery (PCA) is a well-known method to evaluate cerebral blood flow dynamics to changes in neuronal activity in humans $[4,14,16,19,24,27,31,32]$. For this, a visual stimulus is repetitively presented to a subject while cerebral blood flow velocity (CBFV) is measured using transcranial Doppler sonography in the PCA. The visual stimulus may consist of e.g. alternating checkerboards, reading a newspaper or watching a movie. Aligned to the start of each stimulus several responses are averaged to enhance signal to noise ratio. In this way transient effects of increased metabolic demand due to enhanced neuronal activity in the visual cortex can be evaluated.

Another mechanism controlling cerebral blood flow is dynamic cerebral autoregulation (dCA) [2], which continuously adjusts cerebral blood flow with respect to changes in cerebral perfusion pressure. Three different mechanisms are thought to contribute to cerebral autoregulation: metabolic, myogenic and neurogenic regulation [1]. Metabolic regulation adjusts blood flow to both metabolic demand and oxygen supply. Myogenic regulation is the effect of transmural blood pressure changes on vascular smooth muscle tension to keep blood flow constant and neurogenic regulation is the control of the vascular smooth muscle tension through action of the autonomic nervous system.

Because changes in arterial blood pressure (ABP), directly or via dCA may influence cerebral blood flow velocity, one might expect that these effects are reflected in evoked flow responses as well and therefore should be taken into account. Several investigators $[6,7,12,13,25,26]$ who studied evoked changes in $\mathrm{CBFV}$ also monitored concomitant changes in ABP. Their major conclusion was that the influence of blood pressure on evoked flow responses could be neglected due to the relatively small amplitude of these changes (one group [13] reported $2 \%)$. However, these investigators often evaluated ABP not on a beat-to-beat basis but time-averaged over periods of 30 to $60 \mathrm{~s}$. Investigators looking at blood pressure on a beat-to-beat level $[7,15,17,23]$ showed significant effects on evoked flow responses. Moody et al [15] showed a significant effect of beat-to-beat ABP on group-averaged evoked flow responses being an increase in ABP after stimulus start followed by a plateau after approximately 5 seconds. Azevedo et al $[3,4]$ also recorded blood pressure simultaneously with NVC testing but do not report an effect of blood pressure on NVC nor an effect of the stimulus on blood pressure. Using an autoregressive model Panerai et al [17] recently showed that about $20 \%$ of CBFV variance could be explained by ABP in experiments while performing a motor task. It could well be possible that visual stimuli also evoke transient 
blood pressure responses. Furthermore, spontaneous blood pressure changes that evoke a CA response can result in cerebral blood flow variations that may not be negligible after averaging multiple responses for an averaged NVC response. Therefore, the effect of beat-to-beat blood pressure related variation of CBFV in the averaged NVC response needs to be investigated in more detail.

In this study two approaches of quantifying the visually evoked flow response will be compared: first evaluating the flow response as if exclusively evoked by the stimulus and second supposing the flow response to be the addition of on the one hand the stimulus evoked NVC response and on the other the flow resulting from the autoregulatory response due to blood pressure changes. To quantify the stimulus evoked NVC shape a second order linear model (RG) was used introduced by Rosengarten et al [20, 21]. This model was adapted from a model for cerebral autoregulation developed by Tiecks et al [29]. The RG output signal is fitted to the measured NVC response while the input is a zero-to-one step function (zero : visual stimulus off, 1 : visual stimulus on). The RG model quantifies the dynamics of the visual evoked response with four model parameters namely gain $\mathrm{K}$, attenuation $\zeta$, natural frequency $\omega$ and rate time $\mathrm{T}_{\mathrm{v}}$. For cerebral autoregulation we use the original Tiecks model with system's input cerebral perfusion pressure and cerebral blood flow velocity as output.

The aim of this study is to investigate the effect of blood pressure changes via the action of cerebral autoregulation on visual evoked flow responses and eventually to correct the responses for these effects. The recorded CBFV response is assumed to be the linear sum of the blood flow velocity response evoked by the visual stimulus and flow velocity changes due to blood pressure variations under dCA control.

Consequently, two different models will be used to fit the recorded CBFV response. The first only consists of the original RG stimulus response model quantifying the $N V C$ response. The second $\mathrm{RG}_{\mathrm{CA}}$ model, shown in figure 1 , adds to the output of the RG model a CBFV contribution from the CA model based on a beat-to-beat $A B P$ input. Fitting the $\mathrm{RG}_{\mathrm{CA}}$ model to the visual evoked flow response, may result in different RG part parameters compared to the single RG model parameters, since part of the output variance will be explained fromblood pressure related $C B F V$ changes. We hypothesize total fit error is less for the $R G_{C A}$ model compared to the RG model and blood pressure corrected NVC responses result in increased parameter precision. 


\section{Methods}

NVC measurements were evaluated in a heterogeneous population of two separate studies consisting of 21 female subjects who had experienced preeclampsia [14], 15 Alzheimer's disease patients, 17 subjects with mild cognitive impairment and 18 healthy elderly [9]. The Institutional Review Board of the MUMC approved both studies from which data is used and all subjects gave written informed consent. Demographics of all subjects are shown in table 1. All experiments were performed in a quiet room while the subjects laid down with the head slightly tilted. During the experiment the blood flow velocity in the left posterior cerebral artery (P2-segment) and the right middle cerebral artery was measured using a Multidop X4 Doppler device (DWL, Sipplingen, Germany). The two 2-Mhz Doppler probes were mounted on a head band. Continuous arterial blood pressure was recorded at the right hand index or middle finger using a non-invasive cuff device (Task Force Monitor, CN Systems, Austria). The electrocardiogram (ECG) was recorded to facilitate calculation of beat-to-beat ABP and CBFV.

Table 1 Patient demographics

\begin{tabular}{lcccc}
\hline & $\begin{array}{c}\text { Pre- } \\
\text { eclampsia }\end{array}$ & $\begin{array}{c}\text { Alzheimer's } \\
\text { disease }\end{array}$ & $\begin{array}{c}\text { Mild cogni- } \\
\text { tive impaired }\end{array}$ & $\begin{array}{c}\text { Healthy } \\
\text { elderly }\end{array}$ \\
\hline $\mathrm{n}$ & 21 & 15 & 17 & 18 \\
Age [years] & $29(4)$ & $72(7)$ & $70(7)$ & $70(7)$ \\
Male/Female & $0 / 21$ & $7 / 8$ & $11 / 6$ & $8 / 10$ \\
Systolic BP & $123(13)$ & $139(11)$ & $133(11)$ & $134(15)$ \\
{$[$ mmHg] } & & & & \\
Diastolic BP & $83(9)$ & $91(8)$ & $86(10)$ & $88(12)$ \\
{$[$ mmHg] } & & & & \\
\hline
\end{tabular}

To evoke transient cerebral blood flow changes a visual stimulation paradigm was used consisting of at least 10 repetitive episodes of 40 seconds of visual stimulation ("On"), i.e. watching a colored cartoon movie alternated with 20 seconds of black screen ("Off"). During the "Off" period the subjects were instructed to close their eyes. The transition between phases was indicated by an audible tone.

Based on ECG the software automatically detected cardiac cycles and for each beat averages were determined for cerebral blood flow velocity (mCBFV) and blood pressure (mBP). Also the systolic values sCBFV and sBP were taken for each cardiac cycle. All further data analysis was performed for both mean and 
systolic values, but for simplicity only mean will be mentioned. To create equidistant time series for flow velocity and blood pressure repetitively 3 rd order polynomial functions were fitted to 4 subsequent $\mathrm{mBP}$ and $\mathrm{mCBFV}$ beat-to-beat values which were then resampled at $5 \mathrm{~Hz}[8]$.

To correct for unknown TCD insonation angles and to allow inter-individual comparison, absolute blood flow velocity was transformed into changes relative to the value at the onset of stimulation. Also for blood pressure this transform to relative changes was performed. Subsequently, to suppress non stimulus related changes, ensemble averages over all stimulation cycles were calculated.

For each subject the ensemble average mCBFV during the "On" phase of the stimulation cycle is fitted as the output signal of two different control systems. First, only the visual evoked response RG model was applied. Second, in order to incorporate the effect of blood pressure on the $\mathrm{mCBFV}$ response, the averaged blood pressure response was used as a second input for a new two input - one output model. Parameters of both models were determined by a least square fitting technique using Matlab's lsqcurvefit function (Levenberg-Marquardt algorithm) minimizing the fit error between the averaged mCBFV response and the model output. The RG model was described by the second order proportional-derivative model as proposed by Rosengarten et al [21]. The transfer function in the Laplace domain is given in equation 1 where $\omega$ represents an undamped natural frequency, $\zeta$ a damping ratio, $T_{v}$ a rate time and $K$ a gain.

$$
H_{R G}=\frac{K \cdot\left(1+T_{V} \cdot s\right)}{\frac{s^{2}}{\omega^{2}}+2 \cdot \frac{\zeta}{\omega} \cdot s+1}
$$

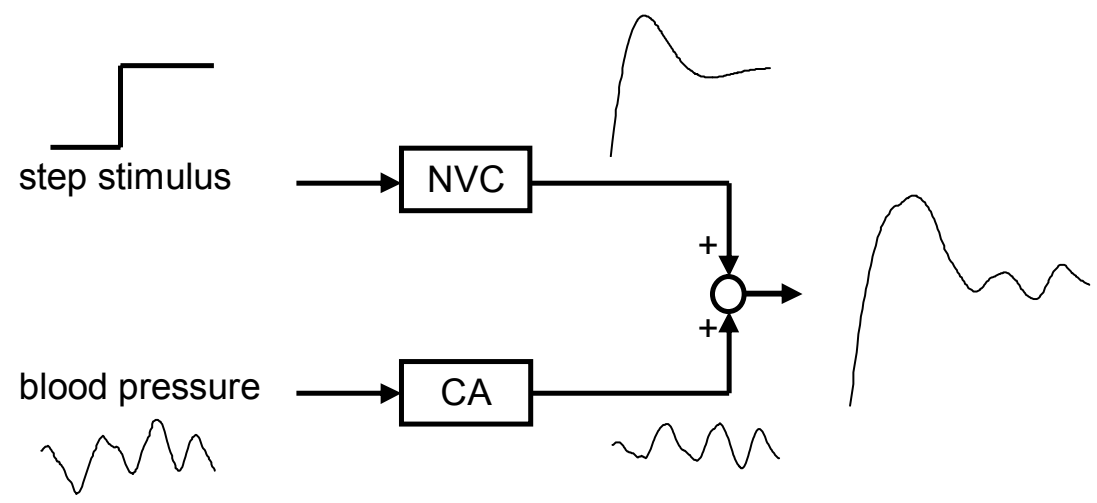

Figure 12 -input 1-output $\mathrm{RG}_{\mathrm{CA}}$ model combining $\mathrm{NVC}$ response to stimulus and $\mathrm{CA}$ response to blood pressure. 
In the extended $\mathrm{RG}_{\mathrm{CA}}$ model as shown in figure 1, the output of the NVC model of equation 1 is added to the output of the model for dynamic cerebral autoregulation as described by Tiecks et al [29] describing the transfer from blood pressure to blood flow velocity.

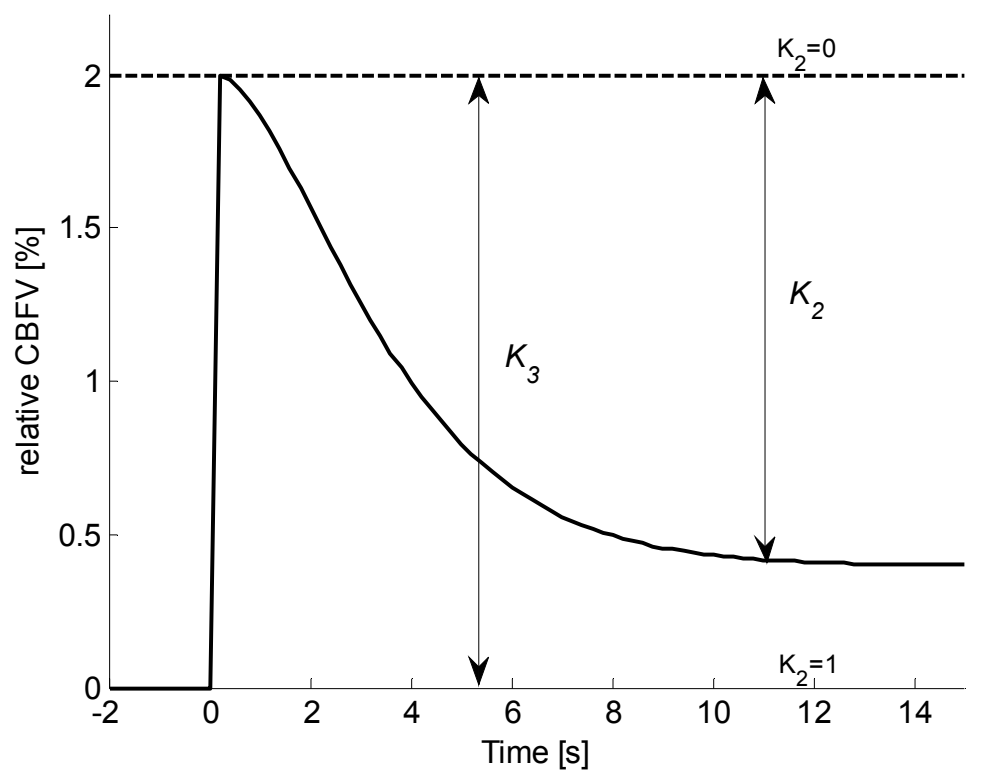

Figure 2 An example step response of dCA model indicating the effect of parameters $\mathrm{K}_{2}$ and $\mathrm{K}_{3}$.

The dynamic cerebral autoregulation transfer can be illustrated by considering the CBFV response to a stepwise increase in blood pressure (figure 2). This increase in blood pressure induces a proportional increase in blood flow velocity. The process of dynamic cerebral autoregulation subsequently adapts cerebrovascular resistance in order to compensate the initial passive increase in blood flow velocity. Previous studies showed, that this process of autoregulation can also be described by a second order control system $[21,29]$ of which the transfer function in the Laplace domain is given by equation 2 .

$$
H_{C A}=K_{3} \cdot\left(1-\frac{K_{2}}{(T \cdot S)^{2}+2 \cdot T \cdot D \cdot S+1}\right)
$$

$\mathrm{K}_{3}$ represents an overall proportional gain and $\mathrm{K}_{2}$ indicates till what extent $C A$ is able to regulate blood flow velocity. A value for $\mathrm{K}_{2}$ of 0 means no active autoregulation, a value of 1 fully working autoregulation. The time course of blood flow 
regulation is described by parameters $\mathrm{T}$ for oscillation time and $\mathrm{D}$ for damping ratio. K2 equals the parameter $\mathrm{K}$ from Tiecks et al [29] and T and D are identical to their parameters $\mathrm{T}$ and $\mathrm{D}$.

Eventually, fitting the VEFR reponses results in the parameters $K, T_{v}, \omega$ and $\zeta$ for the $\mathrm{RG}$ model and additionally in $\mathrm{K}_{3}, \mathrm{~K}_{2}, \mathrm{~T}$ and $\mathrm{D}$ for the $\mathrm{RG}_{\mathrm{CA}}$ model. From the step response function of the $\mathrm{RG}_{\mathrm{CA}}$ model also the autoregulatory index ARI can be estimated [29]. Because, in the traditional RG model usually systolic values for cerebral blood flow velocity are used, results will be reported both for mean and systolic values of blood pressure and cerebral blood flow velocity.

\section{Parameter reliability}

The Matlab routine used to estimate the NVC parameters computes the Jacobian matrix which can be used to quantify the reliability of the parameter estimates. The Jacobian $(J)$ contains all first-order partial derivatives $\left(\partial \mathrm{p}_{\mathrm{i}} / \partial \mathrm{MSD}_{\mathbf{j}} \mathrm{i}=1 . . .4\right)$ of the parameters $p_{i}$ with respect to the residual vector MSD, which is the mean squared difference between fit and measured response. In case of the 4 parameter RG model an NVC response (stimulus on duration of 40 seconds at $5 \mathrm{~Hz}$ ) yields a 4 by $201 \mathbf{J}$ matrix. Together with the MSD, the 4 by 4 covariance matrix of the parameter coefficient estimates (Cov) is calculated according to equation 3.

$\operatorname{Cov}=\mathbf{M S D}^{*}\left(\mathbf{J}^{\mathrm{T} *} \mathbf{J}\right)^{-1}$

with $\mathbf{J}^{\mathrm{T}}$ the transposed Jacobian Matrix.

The $95 \%$ confidence bounds (C) for the fitted parameters are now given by equation 4.

$\mathbf{C}=\mathbf{p} \pm \mathrm{t}^{*} \sqrt{ } \mathbf{S}$

$\mathbf{p}$ is the vector of the 4 fitted Rosengarten parameters, $t$ depends on the confidence level and is computed using the inverse of Student's t cumulative distribution function and equals 1.96 for $95 \%$ confidence bounds. $\mathbf{S}$ is a vector of the diagonal elements of the estimated covariance matrix of the fitted parameters. The relative width of the confidence bounds $\left(\Delta \mathrm{C}_{\mathrm{p}}\right)$, expressed as a percentage of the estimated parameter, is a measure for the precision of the parameter estimation and can be used for sensitivity analysis, i.e. when confidence bounds are 
wide the fit error is less dependant on the exact parameter value and consequently the fit is not sensitive to changes in this parameter. Additionally, percentage confidence bounds facilitate comparison of precision between different parameters.

Statistical analysis of differences in NVC parameter values and reliabilities with and without blood pressure correction was performed using a Wilcoxon signrank test. Differences were considered significant for p-values less then 0.01 .
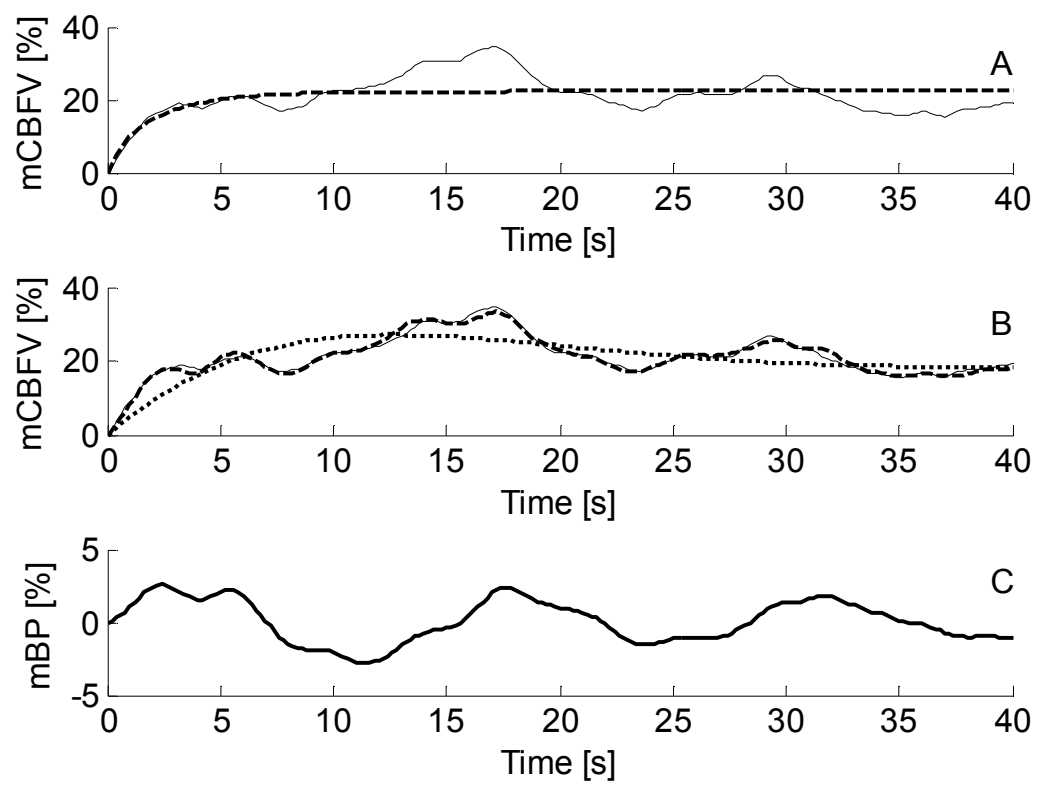

Figure 3 Example of an averaged NVC response (solid A and B) and the fits without (dashed A) and with (dashed B) blood pressure correction. Fitted NVC response with blood pressure corrected model (dotted B). Averaged blood pressure response (solid C). NVC parameters for RG model vs. $R_{\mathrm{CA}}$ model $\omega: 0.67 \rightarrow 0.15 \mathrm{rad} / \mathrm{s}, \zeta: 1.2 \rightarrow 0.6$,

$$
\mathrm{T}_{\mathrm{v}}: 1.4 \rightarrow 12 \mathrm{~s}, \mathrm{~K}: 22 \rightarrow 19
$$

\section{Results}

Data from all subjects were used to evaluate the blood pressure correction of the NVC response. An individual example of an averaged NVC response that is fitted with the two models is shown in figure 3. Figure $3 A$ shows the results for the RG model, figure $3 \mathrm{~B}$ for the $\mathrm{RG}_{\mathrm{CA}}$ model and figure $3 \mathrm{C}$ shows the stimulus averaged blood pressure response. It can be clearly seen that the fitted response of the $\mathrm{RG}_{\mathrm{CA}}$ model (dashed $\mathrm{B}$ ) is better following the averaged evoked flow response 
compared to the $\mathrm{RG}$ fitted response (dashed $\mathrm{A}$ ). In panel $\mathrm{A}$ the dashed line shows the RG fit while the fit of the NVC-part in the $\mathrm{RG}_{\mathrm{CA}}$ model is shown by the dotted line in panel B clearly different from panel A fit, resulting in different NVC parameters. It is clear that part of the variation in the NVC response can be attributed to $\mathrm{ABP}$ variation. It is also clear that in this case the difference in NVC fits with and without blood pressure correction results in different NVC parameters, e.g. attenutation $\zeta$ will be lower after blood pressure correction in this subject due to the less damped response fit.

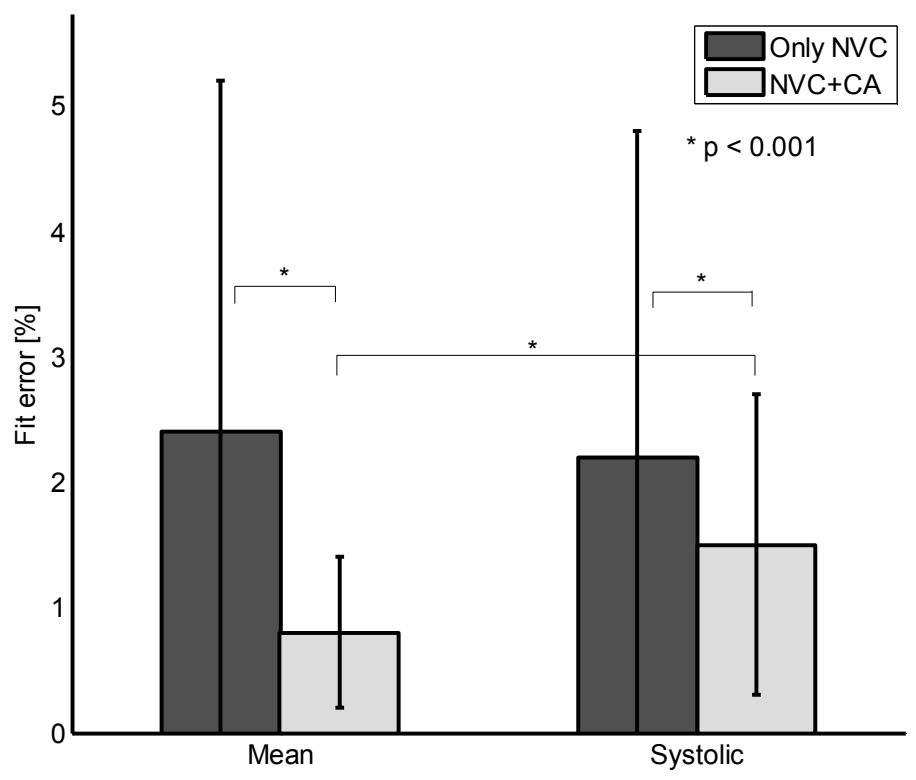

Figure 4 Fit error for mean (left) and systolic (right) NVC responses without (dark) and with (light) blood pressure correction.

Overall results of fit errors are summarized in figure 4 showing significantly decreased fit error for BP-corrected responses $(0.8 \% \pm 0.6)$ compared to noncorrected $(2.4 \% \pm 2.8 \mathrm{p}<0.001)$ for mean blood pressure and flow velocities. This reduction in fit error after blood pressure correction is less pronounced for systolic values: $2.2 \% \pm 2.6$ without and $1.5 \% \pm 1.2$ with BP-correction $(\mathrm{p}<0.001)$. Comparing these fit errors between mean and systolic values did not show a difference before blood pressure correction, but after blood pressure correction the fit error is significantly lower for mean values $(\mathrm{p}<0.001)$. 
Table 2 Median (25-75\%) NVC parameter values for RG and $\mathrm{RG}_{\mathrm{CA}}$ model for averaged mean and systolic $\mathrm{CBFV}$ responses. $\mathrm{CA}$ parameter values for $\mathrm{RG}_{\mathrm{CA}}$ model. $\mathrm{N}=71,{ }^{*} \mathrm{p}<0.01$

\begin{tabular}{lcccc}
\hline & $\begin{array}{c}\text { Before correc- } \\
\text { tion } \\
\text { (mean CBFV) }\end{array}$ & $\begin{array}{c}\text { After correction } \\
\text { (mean CBFV) }\end{array}$ & $\begin{array}{c}\text { Before correc- } \\
\text { tion } \\
\text { (systolic CBFV) }\end{array}$ & $\begin{array}{c}\text { After correc- } \\
\text { tion } \\
\text { (systolic } \\
\text { CBFV) }\end{array}$ \\
\hline$\omega[\mathrm{rad} / \mathrm{s}]$ & $0.18(0.13-0.26)$ & $0.18(0.14-0.24)$ & $0.18(0.14-0.27)$ & $0.19(0.14-0.31)$ \\
$\zeta$ & $0.78(0.57-1.2)$ & $0.74(0.56-1.0) *$ & $0.76(0.63-1.4)$ & $0.81(0.59-1.2)$ \\
$\mathrm{T}_{\mathrm{v}}[\mathrm{s}]$ & $8(3-27)$ & $7(3-17)$ & $7(3-18)$ & $6(3-14)$ \\
$\mathrm{K}_{\mathrm{n}}$ & $23(17-31)$ & $25(18-31)$ & $23(18-29)$ & $23(18-30)$ \\
$\mathrm{K}_{3}$ & & $2.4(1.7-3.6)$ & & $1.8(1.0-2.7)$ \\
$\mathrm{K}_{2}$ & & $0.98(0.63-1.0)$ & & $1.0(0.81-1.0)$ \\
$\mathrm{T}[\mathrm{s}]$ & & $1.3(0.97-1.8)$ & & $1.1(0.68-2.0)$ \\
$\mathrm{D}$ & & $0.58(0.5-1.3)$ & & $0.5(0.5-0.61)$ \\
$\mathrm{ARI}$ & & $5.4(4.6-6.3)$ & & $6.2(4.5-8.2)$ \\
\hline
\end{tabular}

Table 2 shows group median NVC parameters before and after blood pressure correction. It also shows median CA parameter values for the blood pressure corrected model. No significant differences in NVC parameters were found except for $\zeta$ (mean). Blood pressure correction resulted in a small but significant decrease of median $\zeta$ (25-75 percentiles) from 0.8 (0.6-1.2) without to $0.7(0.6-1.0)$ $(\mathrm{p}<0.01)$ with correction. However if average parameter values do not change significantly, individual values may have changed. To show individual parameter changes Bland-Altman plots are shown in figure 5. In each subplot the difference between uncorrected and corrected values are plotted against the average of both for each of the four parameters $\omega(\mathrm{A}), \zeta(\mathrm{B}), \mathrm{Tv}(\mathrm{C})$ and $\mathrm{K}(\mathrm{D})$. The crosses represent estimated parameters on mean and the circles on systolic evoked responses. These plots show the number of parameters that are increased after correction roughly equal the amount of parameters that are decreased after blood pressure correction. 

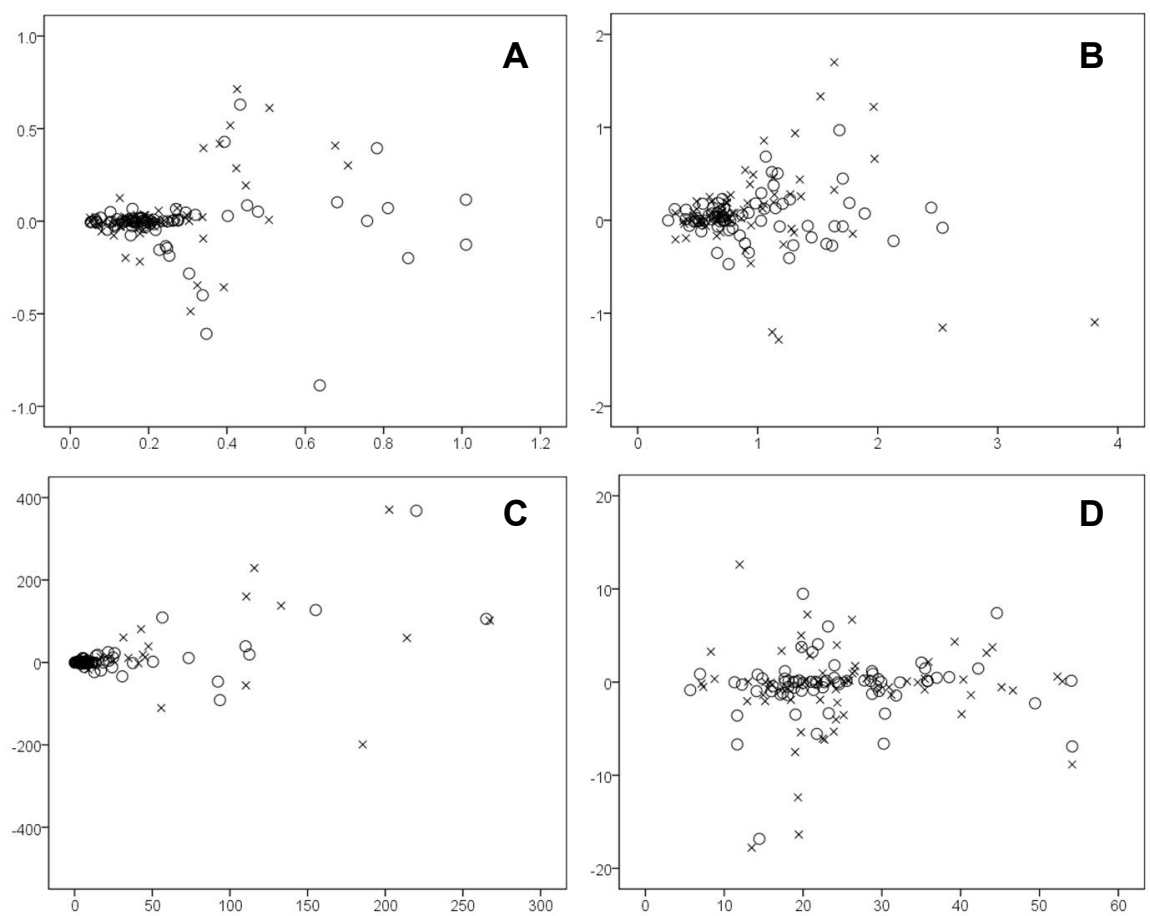

Figure 5 Bland-Altman plots showing the difference (y-axis) against the average ( $\mathrm{x}$ -

axis) of uncorrected and corrected parameter values for mean $(\mathrm{X})$ and systolic $(\mathrm{O})$ NVC responses for the parameters $\omega(A), \zeta(B), T_{v}(C)$ and $K(D)$.

To determine whether a parameter value for an individual measurement changed significantly after blood pressure correction for each measurement for each parameter 95\% confidence bounds of parameter estimation were calculated using equation 4. Per individual measurement parameters with non-overlapping confidence bounds, being a strict criterion, are considered significantly different. Table 3 shows for each NVC parameter the percentage of $R G$ and $\mathrm{RG}_{\mathrm{CA}}$ model estimations for which $95 \%$ confidence bounds do not overlap and therefore changed significantly after correction for blood pressure. 
Table 3 Percentage of parameters that become significantly different after blood pressure correction based on $95 \%$ confidence bounds of parameter estimation. $\mathrm{N}=71$

$$
\begin{gathered}
\text { Percentage parameters changed after blood } \\
\text { pressure correction }
\end{gathered}
$$

\begin{tabular}{lcc}
\hline & mean CBFV & systolic CBFV \\
\hline$\omega[\mathrm{rad} / \mathrm{s}]$ & $28 \%$ & $15 \%$ \\
$\zeta$ & $33 \%$ & $17 \%$ \\
$\mathrm{~T}_{\mathrm{v}}[\mathrm{s}]$ & $25 \%$ & $18 \%$ \\
$\mathrm{~K}$ & $41 \%$ & $31 \%$ \\
\hline
\end{tabular}

Parameter precision was expressed by the relative confidence bound width $C_{P}$. Table 4 shows the median $\left(25^{\text {th }}-75^{\text {th }}\right.$ percentile $)$ values of $C_{p}$ for each of the 4 NVC model parameters. $C_{P}$ values after blood pressure correction are significantly lower $(\mathrm{p}<0.005)$ for all parameters for both mean and systolic data which means that blood pressure correction reduces parameter uncertainty. So, despite the fact that the group averaged parameter values hardly differ between uncorrected and corrected estimation, parameters are estimated more precisely when blood pressure correction is performed.

In case the confidence bounds of a parameter enclose zero value, the precision of the parameter estimate is so poor that basically the parameter cannot be estimated and should be considered unreliable. Table 5 shows the percentage of unreliable parameters without and with blood pressure correction. For the parameter values of $\omega, \zeta$ and $\mathrm{T}_{\mathrm{v}}$ determined on the mean CBFV response a significantly lower fraction $(\mathrm{p}<0.01)$ of unreliable parameters occurs with compared to without blood pressure correction. For $\mathrm{K}$ the improvement is less pronounced possibly due to the already narrow confidence bounds listed in table 4 .

Table 4 Distribution of the $\Delta \mathrm{C}_{\mathrm{P}}$ values of each NVC model parameter before and after correction are displayed as median, $25^{\text {th }}$ and $75^{\text {th }}$ percentile values. $\mathrm{N}=71,{ }^{*} \mathrm{p}<0.005$

\begin{tabular}{lcccc}
\hline & $\begin{array}{c}\text { Before } \\
\text { correction } \\
(\text { mean CBFV) }\end{array}$ & $\begin{array}{c}\text { After } \\
\text { correction } \\
(\text { mean CBFV) }\end{array}$ & $\begin{array}{c}\text { Before } \\
\text { correction } \\
\text { (systolic CBFV) }\end{array}$ & $\begin{array}{c}\text { After } \\
\text { correction } \\
(\text { systolic CBFV) }\end{array}$ \\
\hline$\omega[\mathrm{rad} / \mathrm{s}]$ & $16 \%(7-57)$ & $6 \%(3-22)^{*}$ & $14 \%(8-62)$ & $12 \%(6-34)^{*}$ \\
$\zeta$ & $11 \%(7-35)$ & $6 \%(3-10)^{*}$ & $12 \%(7-45)$ & $10 \%(6-22)^{*}$ \\
$\mathrm{~T}_{\mathrm{v}}[\mathrm{s}]$ & $37 \%(22-257)$ & $18 \%(12-60)^{*}$ & $43 \%(23-148)$ & $36 \%(19-97)^{*}$ \\
$\mathrm{~K}$ & $2 \%(1-11)$ & $1 \%(1-3)^{*}$ & $2 \%(1-6)$ & $1 \%(1-4)^{*}$ \\
\hline
\end{tabular}


Table 5 Percentage of unreliable NVC parameter estimates. $N=71,{ }^{*} \mathrm{p}<0.01$

\begin{tabular}{lcccc}
\hline & $\begin{array}{c}\text { Before } \\
\text { correction } \\
\text { (mean CBFV) }\end{array}$ & $\begin{array}{c}\text { After } \\
\text { correction } \\
\text { (mean CBFV) }\end{array}$ & $\begin{array}{c}\text { Before } \\
\text { correction } \\
\text { (systolic CBFV) }\end{array}$ & $\begin{array}{c}\text { After } \\
\text { correction } \\
\text { (systolic CBFV) }\end{array}$ \\
\hline$\omega[\mathrm{rad} / \mathrm{s}]$ & $17 \%$ & $3 \%^{*}$ & $13 \%$ & $6 \%$ \\
$\zeta$ & $13 \%$ & $3 \%^{*}$ & $8 \%$ & $4 \%$ \\
$\mathrm{~T}_{\mathrm{v}}[\mathrm{s}]$ & $34 \%$ & $15 \% *$ & $31 \%$ & $23 \%$ \\
$\mathrm{~K}$ & $15 \%$ & $10 \%$ & $8 \%$ & $7 \%$ \\
\hline
\end{tabular}

\section{Discussion}

The averaged NVC response can be described more precisely when including cerebral autoregulation in a model of neurovascular coupling. The fit errors for the $\mathrm{RG}_{\mathrm{CA}}$ model with blood pressure correction are significantly lower compared to the RG model. This holds for both averaged responses of mean and systolic CBFV signals, whereas fit error reduction is larger for the mean values. Before blood pressure correction no difference exists between fit errors for mean and systolic values, but after correction fit errors for mean CBFV values are significantly lower compared to systolic fit errors. Blood pressure correction is more effective for averaged NVC responses based on mean CBFV values. This might be explained by the fact that the mechanism of cerebral autoregulation is basically controlling mean cerebral blood flow, which is more comparable with mean CBFV than with systolic CBFV values. Rosengarten et al compared systolic and diastolic $\mathrm{CBFV}$ responses [22] and concluded that both could be used to determine parameters of the control system model, although the values differed for the two conditions. They preferred using the systolic response, because this would be more robust and less susceptible to artifacts. They did not report results using mean CBFV responses.

Comparison of NVC parameters for the whole group did not show significant differences for the parameters except for a small difference in $\zeta$ for mean CBFV values. This suggests that although fit errors are significantly lower after blood pressure correction, NVC parameter values are not affected. However, parameters can be both increased or decreased after blood pressure correction which could result in no average change, whereas individual parameters change significantly. This was confirmed by the absolute differences between corrected and uncorrected parameters as shown in figure 5. To investigate if individual parameters changed significantly their changes were evaluated based on non- 
overlapping confidence bounds of parameter estimations. This shows that for mean CBFV parameters changed in $25-41 \%$ of the cases and for systolic CBFV responses in $15-31 \%$ of cases. Individual parameters therefore do change significantly and this could be of influence on findings earlier presented on NVC parameters.

Evaluating the precision of parameter estimations by the value of $\mathrm{C}_{\mathrm{P}}$ shows a significant decrease in confidence bound width for all NVC parameters. This means parameters are estimated more precisely leading to less intra-individual variation. This could enhance the power to detect differences between different patient groups, but could also lead to less pronounced effects shown with analyses performed on uncorrected responses. But, showing that blood pressure variation via cerebral autoregulation can have a significant improvement on NVC response fits advocates incorporation of cerebral autoregulation in the model used to fit these responses.

Recently, several investigators evaluated reproducibility of TCD-based evaluation of cerebral hemodynamic function $[5,8,10,23,30]$. Good reproducibility is of key importance for the clinical application of these tests, especially when used to evaluate therapeutic interventions or monitor disease course. Combining mechanisms such as neurovascular coupling, cerebral autoregulation and $\mathrm{CO}_{2}$ reactivity might help to disentangle the specific contributions of each mechanism and improve the reproducibility of the estimated parameters describing each process. Panerai et al [17] presented a promising approach using autoregressive models whereas we combined two known models for neurovascular coupling and cerebral autoregulation. Both are attempts for more reliable parameter estimation and reduction of unexplained variance. We also measured end-tidal $\mathrm{CO}_{2}$-data but did not yet use this as an extra model input. A different approach might be to build a more physiological based model that consists of components for different regulatory mechanisms: metabolic, neurogenic and myogenic. Payne [18] described such a model, which is a combination of a hemodynamic model and different feedback mechanisms adjusting arterial compliance and resistance. A similar approach is evaluated by Spronck [28].

In this study cerebral autoregulation is modeled on the stimulus averaged responses of blood pressure and cerebral blood flow velocity. However, cerebral autoregulation is basically not a visual stimulus related mechanism but is continuously reacting to blood pressure variability. Therefore it might be considered to evaluate the effect of cerebral autoregulation on the original non averaged raw signals. This needs to be investigated in further research. 


\section{Limitations}

With TCD only blood flow velocity can be measured and no absolute flow due to unknown blood vessel diameter. Because NVC responses are converted into relative responses this is no issue, as long as the vessel diameter remains constant during the evoked responses. PCA diameter is approximately $2.5 \mathrm{~mm}$. Huber et al [11] showed via angiography that cerebral arteries with a diameter of at least 2.5 mm do not show changes in diameter with hypercapnia, hyperventilation, hypertonic glucose and administration of papaverine. We assumed also visually evoked flow enhancement does not influence PCA diameter.

The combined $\mathrm{RG}_{\mathrm{CA}}$ model used, which is an addition of an NVC model and a CA model, might not be optimal. Both sub-systems, i.e. the NVC and CA system, are modeled with a second order model. For each system individually this was shown to be adequate $[20,29]$. However, if both systems work (partly) through the same mechanisms, which could well be the case, the combined system might be over dimensioned and less parameters might be necessary to describe the evoked response with the same precision. Currently the combined $\mathrm{RG}_{\mathrm{CA}}$ model consists of eight parameters. This is the same amount as the ARMA model of Panerai et al [17], but as already mentioned, they also incorporated the influence of $\mathrm{CO}_{2}$ from a third input. Improvements might be achieved by stepwise downsizing the model and evaluating if parameter reliability improves with a more simpler model. However, what is clear from our results is that parameter reliability improved in the $\mathrm{RG}_{\mathrm{CA}}$ model compared to the $\mathrm{RG}$ model.

Our data consists of a heterogeneous set from different patient groups and healthy elderly. Our aim was to study the influence of blood pressure related changes in CBFV in relation to quantification of NVC, which is a purely methodological approach. Since all data analysis is linear, all data should equally be effected by these methodological differences.

\section{Conclusion}

Blood pressure correction of NVC responses by including cerebral autoregulation in model fitting of averaged VEFR responses results in significantly lower fit errors. Blood pressure correction is more effective when mean instead of systolic CBFV responses are used. A substantial amount of NVC parameters change after blood pressure correction resulting in more narrow confidence bounds and due to that into more reliably estimates. Measurement and quantification of NVC should include beat-to-beat blood pressure measurement. 
Blood pressure corrected evoked flow responses

\section{Acknowledgements}

The authors thank Prof. R.B. Panerai (University of Leicester, UK) for his valuable contributions to the manuscript.

\section{Grants}

Research partly supported by: Internationale Stichting Alzheimer Onderzoek (ISAO) grant \# 06518 to JR.

\section{Disclosures}

No conflict of interest, financial or otherwise, are declared by the author(s).

\section{Authors contributions}

Author contributions: J.R., W.M., E.M. and E.G., conception and design of research; G.B., E.M. and E.G. analysed data; E.M. performed experiments; G.B. and E.G. drafted manuscript; J.R., W.M. and E.G. edited and revised manuscript; G.B., J.R., W.M., E.M. and E.G. approved final manuscript; G.B. and E.G. prepared figures. 


\section{References}

1. Aaslid R. Cerebral autoregulation and vasomotor reactivity. Front Neurol Neurosci 21: 216-228, 2006.

2. Aaslid R, Lindegaard KF, Sorteberg W, and Nornes H. Cerebral autoregulation dynamics in humans. Stroke 20: 45-52, 1989.

3. Azevedo E, Rosengarten B, Santos R, Freitas J, and Kaps M. Interplay of cerebral autoregulation and neurovascular coupling evaluated by functional TCD in different orthostatic conditions. J Neurol 254: 236-241, 2007.

4. Azevedo E, Santos R, Freitas J, Rosas M-J, Gago M, Garrett C, and Rosengarten B. Deep brain stimulation does not change neurovascular coupling in non-motor visual cortex: An autonomic and visual evoked blood flow velocity response study. Parkinsonism es Related Disorders 16 : 600-603, 2010.

5. Brodie FG, Atkins ER, Robinson TG, and Panerai RB. Reliability of dynamic cerebral autoregulation measurement using spontaneous fluctuations in blood pressure. Clin Sci (Lond) 116: 513-520, 2009.

6. Cupini LM, Matteis M, Troisi E, Sabbadini M, Bernardi G, Caltagirone $\mathrm{C}$, and Silvestrini M. Bilateral simultaneous transcranial Doppler monitoring of flow velocity changes during visuospatial and verbal working memory tasks. Brain 119: 1249-1253, 1996.

7. Duschek S, Heiss H, Schmidt MF, Werner NS, and Schuepbach D. Interactions between systemic hemodynamics and cerebral blood flow during attentional processing. Psychophysiology 47: 1159-1166, 2010.

8. Gommer E, Shijaku E, Mess W, and Reulen J. Dynamic cerebral autoregulation: different signal processing methods without influence on results and reproducibility. Med Biol Eng Comput 1-8, 2010.

9. Gommer ED, Martens EG, Aalten P, Shijaku E, Verhey FR, Mess WH, Ramakers IH, and Reulen JP. Dynamic Cerebral Autoregulation in Subjects with Alzheimer's Disease, Mild Cognitive Impairment, and Controls: Evidence for Increased Peripheral Vascular Resistance with Possible Predictive Value. J Alzheimers Dis 30: 805-813, 2012.

10. Hu K, Peng CK, Czosnyka M, Zhao P, and Novak V. Nonlinear assessment of cerebral autoregulation from spontaneous blood pressure and cerebral blood flow fluctuations. Cardiovasc Eng 8: 60-71, 2008.

11. Huber P, and Handa J. Effect of contrast material, hypercapnia, hyperventilation, hypertonic glucose and papaverine on the diameter of the 
cerebral arteries. Angiographic determination in man. Invest Radiol 2:1732, 1967.

12. Jorgensen LG, Perko G, Payne G, and Secher NH. Effect of limb anesthesia on middle cerebral response to handgrip. American Journal of Physiology - Heart and Circulatory Physiology 264: H553-H559, 1993.

13. Klingelhofer J, Matzander G, Sander D, Schwarze J, Boecker H, and Bischoff C. Assessment of Functional Hemispheric Asymmetry by Bilateral Simultaneous Cerebral Blood Flow Velocity Monitoring. J Cereb Blood Flow Metab 17: 577-585, 1997.

14. Martens EG, Peeters LL, Gommer ED, Mess WH, van de Vosse FN, Passos VL, and Reulen JP. The visually-evoked cerebral blood flow response in women with a recent history of preeclampsia and/or eclampsia. Ultrasound Med Biol 35: 1-7, 2009.

15. Moody M, Panerai RB, Eames PJ, and Potter JF. Cerebral and systemic hemodynamic changes during cognitive and motor activation paradigms. Am J Physiol Regul Integr Comp Physiol 288: R1581-1588, 2005.

16. Panerai RB, Moody M, Eames PJ, and Potter JF. Dynamic cerebral autoregulation during brain activation paradigms. Am J Physiol Heart Circ Physiol 289: H1202-1208, 2005.

17. Panerai RB, Salinet ASM, and Robinson TG. Contribution of arterial blood pressure and $\mathrm{PaCO} 2$ to the cerebrovascular responses to motor stimulation. Am J Physiol Heart Circ Physiol 302: H459-H466, 2012.

18. Payne SJ.A model of the interaction between autoregulation and neural activation in the brain. Math Biosci 204: 260-281, 2006.

19. Rosengarten B, Auch D, and Kaps M. Effects of Initiation and Acute Withdrawal of Statins on the Neurovascular Coupling Mechanism in Healthy, Normocholesterolemic Humans. Stroke 38: 3193-3197, 2007.

20. Rosengarten B, Huwendiek O, and Kaps M. Neurovascular coupling and cerebral autoregulation can be described in terms of a control system. Ultrasound Med Biol 27: 189-193, 2001.

21. Rosengarten B, Huwendiek O, and Kaps M. Neurovascular coupling in terms of a control system: validation of a second-order linear system model. Ultrasound Med Biol 27: 631-635, 2001.

22. Rosengarten B, Osthaus S, Auch D, and Kaps M. Effects of acute hyperhomocysteinemia on the neurovascular coupling mechanism in healthy young adults. Stroke 34: 446-451, 2003.

23. Salinet AS, Robinson TG, and Panerai RB. Reproducibility of cerebral and peripheral haemodynamic responses to active, passive and motor 
imagery paradigms in older healthy volunteers: A fTCD study.J Neurosci Methods 206: 143-150, 2012.

24. Schroeter ML, Cutini S, Wahl MM, Scheid R, and Yves von Cramon D. Neurovascular coupling is impaired in cerebral microangiopathy-An event-related Stroop study. Neuroimage 2006.

25. Silvestrini M, Troisi E, Matteis M, Razzano C, and Caltagirone C. Correlations of flow velocity changes during mental activity and recovery from aphasia in ischemic stroke. Neurology 50: 191-195, 1998.

26. Sitzer M, Knorr U, and Seitz RJ. Cerebral hemodynamics during sensorimotor activation in humans. Journal of Applied Physiology 77: 28042811, 1994.

27. Smith EE, Vijayappa M, Lima F, Delgado P, Wendell L, Rosand J, and Greenberg SM. Impaired visual evoked flow velocity response in cerebral amyloid angiopathy. Neurology 71: 1424-1430, 2008.

28. Spronck B, Martens EG, Gommer ED, and van de Vosse FN. A lumped parameter model of cerebral blood flow control combining cerebral autoregulation and neurovascular coupling. Am J Physiol Heart Circ Physiol 303: H1143-1153, 2012.

29. Tiecks FP, Lam AM, Aaslid R, and Newell DW. Comparison of static and dynamic cerebral autoregulation measurements. Stroke 26: 10141019, 1995.

30. van Beek AH, Olde Rikkert MG, Pasman JW, Hopman MT, and Claassen JA. Dynamic Cerebral Autoregulation in the Old Using a Repeated Sit-Stand Maneuver. Ultrasound Med Biol 36: 192-201, 2010.

31. Zaletel M, Strucl M, Pretnar-Oblak J, and Zvan B. Age-related changes in the relationship between visual evoked potentials and visually evoked cerebral blood flow velocity response. Funct Neurol 20:115-120, 2005.

32. Zaletel M, Strucl M, Rodi Z, and Zvan B. The relationship between visually evoked cerebral blood flow velocity responses and visualevoked potentials. Neuroimage 22: 1784-1789, 2004. 


\section{Chapter 6}

A new method for assessment of dynamic cerebral autoregulation and cerebral carbon dioxide reactivity during cardiopulmonary bypass

E E Ševerdija, E D Gommer, P W Weerwind, J P H Reulen, W H Mess, J G Maessen. A new method for assessment of dynamic cerebral auto-regulation and cerebral carbon dioxide reactivity during cardiopulmonary bypass. Submitted for publication 


\section{Abstract}

Coronary artery bypass graft (CABG) surgery is associated with significant morbidity and mortality. Worsened control of cerebral blood flow, due to hypertension, diabetes or other conditions impose patients peri-operatively to increased risk of neurological complications. However, evaluation of cerebral hemodynamics is not common practice. We present a technique to evaluate dynamic cerebral autoregulation (dCA) and cerebral carbon dioxide reactivity $\left(\mathrm{CO}_{2} \mathrm{R}\right)$ during normothermic nonpulsatile cardiopulmonary bypass (CPB). The technique uses continuous recording of invasive arterial blood pressure, middle cerebral artery blood flow velocity through transcranial Doppler sonography, absolute cerebral tissue saturation via near-infrared spectroscopy, in-line arterial carbon dioxide and pump flow measurement. dCA during CPB is estimated by transfer function analysis based on the response to blood pressure variation induced by cyclic $6 / \mathrm{min}$ changes of indexed pump flow from 2.0 to 2.4 up to 2.8 $1 / \mathrm{min} / \mathrm{m}^{2} . \mathrm{CO}_{2} \mathrm{R}$ is calculated from recordings of both cerebral blood flow velocity and cerebral tissue oxygenation. In 37 patients during $\mathrm{CPB}$ we tested the feasibility of our technique to estimate $\mathrm{dCA}$ and $\mathrm{CO}_{2} \mathrm{R}$ at hypocapnia $\left(\mathrm{p}_{\mathrm{a}} \mathrm{CO}_{2}=30\right.$ $\mathrm{mmHg})$, normocapnia $\left(\mathrm{p}_{\mathrm{a}} \mathrm{CO}_{2}=40 \mathrm{mmHg}\right)$, and hypercapnia $\left(\mathrm{p}_{\mathrm{a}} \mathrm{CO}_{2}=50\right.$ $\mathrm{mmHg})$. dCA phase decreased significantly $(\mathrm{p}<0.01)$ with increasing $\mathrm{p}_{\mathrm{a}} \mathrm{CO}_{2}$ from hypocapnia $(0.58 \pm 0.3 \mathrm{rad})$ to normocapnia $(0.31 \pm 0.2 \mathrm{rad})$ and hypercapnia $(0.1$ $\pm 0.1 \mathrm{rad}$ ). Also gain decreased ( $\mathrm{p}<0.01)$ with increasing $\mathrm{p}_{\mathrm{a}} \mathrm{CO}_{2}$ from hypocapnia $(3.4 \pm 2 \% / \mathrm{mmHg})$ to normocapnia $(2.2 \pm 0.9 \% / \mathrm{mmHg})$ and hypercapnia $(1.4 \pm$ $0.4 \% / \mathrm{mmHg}$ ). $\mathrm{CO}_{2} \mathrm{R}$ is preserved during $\mathrm{CPB}$, but significantly lower $(\mathrm{p}<0.01)$ for hypocapnia $(2.5 \pm 1 \% / \mathrm{mmHg})$ compared to hypercapnia $(5.0 \pm 2 \% / \mathrm{mmHg})$. Studying dynamic cerebral autoregulation and cerebrovascular reactivity during cardiopulmonary bypass is feasible by changing pump flow in a $6 / \mathrm{min}$ rate. Hypercapnia results in impaired dCA. Ongoing and future studies are expected to clarify optimal perfusion strategies for various patient populations scheduled for operations involving cardiopulmonary bypass. 


\section{Introduction}

Coronary artery bypass graft surgery (CABG) is a common operation that is associated with significant morbidity and mortality [41]. Although operative techniques, anaesthesia and cardiopulmonary bypass have improved outcomes over the past 10 years, there is inherent neurological risk associated with CABG. Stroke and postoperative cognitive decline, two major neurological outcomes, may be related to cerebral hypoperfusion during CABG [42]. The frequency of any postoperative cerebral injury has been estimated at between 6 and 28\% for encephalopathy alone. Moreover, $3 \%$ of patients undergoing coronary bypass surgery have been estimated to have serious neurological sequelae (death due to cerebral injury, stroke, transient ischemic attack, or stupor) [40]. Worsened control of cerebral blood flow, due to hypertension, diabetes or other conditions impose patients peri-operatively to increased risk of neurological complications [10]. Despite this, evaluation of cerebral hemodynamics is not common practice.

Cerebral autoregulation $(\mathrm{CA})$ and carbon dioxide reactivity $\left(\mathrm{CO}_{2} \mathrm{R}\right)$ are two important mechanisms for controlling cerebral blood flow (CBF). In healthy humans CBF is controlled over a cerebral perfusion pressure range of 60-150 $\mathrm{mmHg}$ [35] via vasomotor effectors that control cerebrovascular resistance [3]. Dysfunction of CA and impaired $\mathrm{CO}_{2} \mathrm{R}$ before and during $\mathrm{CPB}$ might contribute to neurological morbidity after cardiac surgery [24, 25, 45]. Currently, blood pressure management during $\mathrm{CPB}$ is based on maintaining a minimum mean arterial blood pressure (ABP) of approximately $50 \mathrm{mmHg}$ [44] and is neither monitored nor controlled for adequate function of CA and/or $\mathrm{CO}_{2} \mathrm{R}$.

In general, increasing $\mathrm{ABP}$ out of $\mathrm{CA}$ range may cause hyperperfusion with oedema formation [52], whereas reduction of CBF due to decreased ABP below the autoregulatory range may cause cerebral hypoperfusion and ischemia [35]. There are two animal studies [43, 46] and one in human subjects [11], indicating that $\mathrm{CBF}$ and mean $\mathrm{ABP}$ are not influenced by $\mathrm{CPB}$ indexed pump flow provided mean $\mathrm{ABP}$ is kept within the autoregulatory range. CBF will only decrease proportionally to a decrease in pump flow when mean ABP decreases below the subcritical perfusion pressure, i.e. the lower limit of autoregulation. Oppositely, increasing indexed pump flow leading may lead to hyperperfusion when mean $\mathrm{ABP}$ rises above the upper limit of autoregulation. Thus, individualizing ABP during $\mathrm{CPB}$ to be within a patient's $\mathrm{CA}$ working range might prevent either cerebral hypoperfusion due to low ABP and/or cerebral oedema from high ABP. However, lower and upper limits of autoregulation are not known pre- 


\section{Chapter 6}

operatively. Moreover, it is unethical to change blood pressure over such large ranges other measures are necessary to determine the level of cerebral blood flow regulation. Consequently, dynamic cerebral autoregulation (dCA) should be assessed during $\mathrm{CPB}$.

Instead of determining static lower and upper limits of cerebral autoregulation, $\mathrm{dCA}$ quantifies the response of $\mathrm{CBF}$ to transient and/or periodic changes in $\mathrm{ABP}$. Brady et al [7] have evaluated cerebral blood flow autoregulation continuously during CPB using Transcranial Doppler sonography (TCD) calculating crosscorrelation $(\mathrm{Mx})$ between slow waves $(\mathrm{f}<0.05 \mathrm{~Hz})$ of middle cerebral artery blood flow velocity (CBFV) and mean arterial blood pressure (ABP). Similarly, cerebral oximetry index (COx), recorded through Near-Infrared Spectroscopy (NIRS), was calculated for slow waves of cerebral oximetry and ABP. They show high values of $\mathrm{Mx}$ and $\mathrm{COx}$ during $\mathrm{CPB}$ suggesting impaired cerebral autoregulation. However, they do not mention whether sufficient blood pressure variability occured during CPB to reliably calculate signal cross-correlation. An important point since a patient on $\mathrm{CPB}$ usually shows very little spontaneous $\mathrm{ABP}$ variability. Furthermore, Mx is essentially an intermediate indicator of static CA and dynamic cerebral autoregulation, since it does not take into account the phase relationship in the autoregulatory response [1]. Apart from $\mathrm{Mx}$ we are not aware of any other method evaluating dCA during CPB.

A common approach to quantify dynamic cerebral autoregulation (dCA) is to analyse $\mathrm{ABP}$ oscillations as input and $\mathrm{CBFV}$, measured in the middle cerebral artery (MCA) using TCD, as output signal in the frequency domain using transfer function analysis (TFA) [31, 53]. Under the assumption of a constant MCA diameter $\mathrm{CBFV}$ is linearly related to $\mathrm{CBF}[6,48]$. A positive phase shift is present when $\mathrm{CBFV}$ oscillations precede $\mathrm{ABP}$ oscillations in the low frequency range around $0.1 \mathrm{~Hz}$ and is interpreted as intact dCA [13]. In a previous study in healthy volunteers under normal supine conditions we found average values of phase shift of $0.9 \pm 0.3 \mathrm{rad}[15] . \mathrm{CO}_{2} \mathrm{R}$ is an index of cerebrovascular dilatory capacity to an increase in arterial carbon dioxide $\left(\mathrm{p}_{\mathrm{a}} \mathrm{CO}_{2}\right)$ level, and is defined as the change in $\mathrm{CBF}$ per unit change in $\mathrm{p}_{\mathrm{a}} \mathrm{CO}_{2} \cdot \mathrm{CO}_{2} \mathrm{R}$ is not a dynamic measure but represents the capacity of arterioles to respond to changes in $\mathrm{p}_{\mathrm{a}} \mathrm{CO}_{2} \cdot \mathrm{CO}_{2} \mathrm{R}$ can readily be assessed using TCD [51] or NIRS [47]. Normal $\mathrm{CO}_{2} \mathrm{R}$ is estimated to be $2-3 \%$ decrease in CBF per mmHg decrease in $\mathrm{p}_{\mathrm{a}} \mathrm{CO}_{2}$ between 3-4\% increase per $\mathrm{mmHg}$ increase in $\mathrm{p}_{\mathrm{a}} \mathrm{CO}_{2}$ [9]. Additionally, the $\mathrm{CO}_{2} \mathrm{R}$ measured with NIRS in supine position is found to be $0.36 \pm 0.1$ per $\mathrm{mmHg}$ change in $\mathrm{p}_{\mathrm{a}} \mathrm{CO}_{2}[39$ ]. 
The TFA method for quantifying dCA is applicable only when sufficient variability in blood pressure subsequently is present leading to cerebral blood flow velocity variability. Because during $\mathrm{CPB}$ hardly any variation in $\mathrm{ABP}$ occurs, blood pressure variability could be induced by cyclic changes in pump flow. Diehl et al [14] showed in awake subjects that blood pressure variations can be induced by breathing at a paced rhythm of $6 / \mathrm{min}$. Analogue to this we induced an indexed pump flow change from 2.0 to $2.8 \mathrm{1} / \mathrm{min} / \mathrm{m}^{2}$ during CPB at a frequency of $6 / \mathrm{min}$. The present study was performed to test the $\mathrm{CBFV}$ response to pump flow induced oscillations in $\mathrm{ABP}$, and to evaluate cerebral autoregulation and cerebral carbon dioxide reactivity during normothermic $\mathrm{CPB}$ using nonpulsatile flow. 


\section{Patients and methods}

In this study 37 male patients aged between 43 and 70 years, were investigated who underwent elective CABG surgery using normothermic CPB. The Institutional Review Board of the MUMC approved the study and all subjects gave written informed consent. Patients were excluded if the temporal bone window was inadequate for transcranial ultrasonography. Other exclusion criteria consisted of neurological disorders (e.g. cerebrovascular accident), renal diseases (e.g. renal failure, defined as laboratory tests indicating values of twice the normal values or more [urea $\geq 50 \mathrm{U} / 1$, kreatinine $\geq 170 \mu \mathrm{mol} / 1$ ]), liver diseases (defined as laboratory tests indicating values of twice the normal values or more (ASAT $\geq 50$ $\mathrm{U} / 1$, ALAT $\geq 60 \mathrm{U} / 1, \mathrm{LD} \geq 600 \mathrm{U} / 1$ and $\gamma-\mathrm{GT} \geq 90 \mathrm{U} / \mathrm{l})$ ), severe pulmonary disorders (e.g. chronic obstructive pulmonary disease, emphysema), insulin and non insulin dependent diabetics, severe atherosclerosis of carotid or middle cerebral artery, and participation in an investigational drug trial within the preceding 30 days. Demographics of all subjects are presented in table 1.

Table 1 Patient baseline parameters $(n=37)$, mean (std)

\begin{tabular}{lc}
\hline Age $[$ years] & $61(6)$ \\
Weight $[\mathrm{kg}]$ & $86(11)$ \\
Height $[\mathrm{cm}]$ & $177(6)$ \\
BSA $[\mathrm{m} 2]$ & $2.03(0.16)$ \\
BMI $[\mathrm{kg} / \mathrm{cm} 2]$ & $27.9(4)$ \\
Hct $[\%]$ & $44(3)$ \\
Mean ABP [mmHg] & $88(10)$ \\
Mean CBFV left $[\mathrm{cm} / \mathrm{s}]$ & $43(11)$ \\
Mean CBFV right $[\mathrm{cm} / \mathrm{s}]$ & $42(10)$ \\
Mean SctO2 left $[\%]$ & $72(3)$ \\
Mean SctO2 right $[\%]$ & $70(2)$ \\
\hline
\end{tabular}


General anaesthesia was induced using weight-related dosing of sufentanil and etomidate, and muscle relaxation was achieved with pancuronium bromide. General anaesthesia was maintained during surgery using propofol. An initial dose of heparin, $300 \mathrm{IU} / \mathrm{kg}$ of body weight (Leo Pharmaceutical Products ${ }^{\circledR}$, Ballerup, Denmark), was injected into a central venous catheter before the initiation of CPB. When a target activated clotting time exceeded 400 seconds CPB was started. At the end of $\mathrm{CPB}$ heparin was reversed by protamine chloride (Valeant Pharmaceuticals ${ }^{\circledR}$, Eschborn, Germany) at a 1:1 ratio of the total loading dose before CPB. An extracorporeal circuit optimized (ECCo) for CPB system (Sorin Group Italia ${ }^{\circledR}$, Mirandola, Italy) was used consisting of a revolution disposable centrifugal pump (Sorin Group Italia ${ }^{\circledR}$, Mirandola, Italy), a phosphorylcholine coated $1.1 \mathrm{~m}^{2}$ hollow fiber oxygenator (Sorin Group Italia ${ }^{\circledR}$, Mirandola, Italy), a cell-saving device (Dideco Electa, Sorin Group Italia ${ }^{\circledR}$, Mirandola, Italy), $3 / 8$ " arterial and venous lines and an intermittently isolated hard-shell reservoir (D 790, Sorin Group Italia ${ }^{\circledR}$, Mirandola, Italy).

For dynamic assessment of $\mathrm{CA}$ and cerebral carbon dioxide reactivity during $\mathrm{CPB}, \mathrm{ABP}$, middle cerebral artery blood flow velocity, $\mathrm{p}_{\mathrm{a}} \mathrm{CO}_{2}$, absolute cerebral tissue oxygenation and $\mathrm{CPB}$ pump flow were continuously recorded. $\mathrm{ABP}$ was recorded from an intra-arterial pressure module (Philips Medical ${ }^{\circledR}$, The Netherlands), CBFV recordings of both left and right middle cerebral artery via two $2 \mathrm{MHz}$ TCD probes mounted on a head frame (ST3, Spencer Technologies ${ }^{\circledR}$, Seattle, USA) at a depth between 45 and $56 \mathrm{~mm}$, in-line arterial $\mathrm{p}_{\mathrm{a}} \mathrm{CO}_{2}$ measurement via an optical fluorescence and reflectance-based system (CDI-500, Terumo ${ }^{\circledR}$, Japan), absolute cerebral tissue saturation $\left(\mathrm{SctO}_{2}\right)$ via near-infrared spectroscopy (ForeSight, Casmed ${ }^{\circledR}$, USA) with the electrodes placed on the right and left forehead, whereas pump flow was recorded by an ultrasonic flow monitor (Transonic Systems Europe BV ${ }^{\circledR}$, Maastricht, The Netherlands). All data were sampled at $250 \mathrm{~Hz}$ and stored using an 8-channel acquisition system (IDEEQ, Maastricht Instruments ${ }^{\circledR}$, Maastricht, the Netherlands), and were subsequently down sampled to $5 \mathrm{~Hz}$ by averaging over $0.2 \mathrm{~s}$ intervals.

Similar to Diehl et al (7) who assessed CA in awake patients using triggered 6/min deep breathing to vary cardiac output, our method uses three level cyclic changes of indexed pump flow from a lower level of $2.0 \mathrm{l} / \mathrm{min} / \mathrm{m}^{2}$ to a base level of $2.4 \mathrm{l} / \mathrm{min} / \mathrm{m}^{2}$ and to an upper level of $2.8 \mathrm{l} / \mathrm{min} / \mathrm{m}^{2}$, with a frequency of 6 cycles $/ \mathrm{min}$ ( $2.5 \mathrm{~s}$ per indexed flow rate).

All measurements were performed after $\mathrm{CPB}$ (constant hematocrit, constant arterial blood temperature, constant arterial oxygen tension, and constant pump 
flow) was started, the aorta was cross clamped, cardioplegia was infused and the patient was hemodynamically stabilized. First, $\mathrm{p}_{\mathrm{a}} \mathrm{CO}_{2}$ was set to a hypocapnic level of $30 \mathrm{mmHg}$ and after this was stable for a minute mean CBFV was determined by averaging CBFV over ten seconds. Thereafter, 5 minutes of cyclic changes of indexed pump flow were induced. The flow was manually set to each of the three levels $\left(2.0,2.4\right.$ and $\left.2.81 / \mathrm{min} / \mathrm{m}^{2}\right)$ upon a verbal command indicating the $6 / \mathrm{min}$ rhythm. The 5 minute period was used to evaluate cerebral autoregulation. Subsequently, $\mathrm{p}_{\mathrm{a}} \mathrm{CO}_{2}$ was set to a normocapnic level of $40 \mathrm{mmHg}$ respectively to a hypercapnic level of $50 \mathrm{mmHg}$. At every level of $\mathrm{p}_{\mathrm{a}} \mathrm{CO}_{2}$ first ten seconds of steady flow were used to determine baseline mean CBFV followed by 5 minutes of cyclic changes of pump flow. The mean CBFV data acquired at each of the three levels of $\mathrm{p}_{\mathrm{a}} \mathrm{CO}_{2}$ were used to calculate $\mathrm{CO}_{2} \mathrm{R}$.

\section{Data analysis}

After recording and down sampling of the data, the transfer function yielding coherence, gain and phase shift angle between oscillation in CBFV and ABP was calculated, as described by Gommer et al [17]. The transfer function $H(f)$ was calculated by

$$
H(f)=\frac{S_{x y}(f)}{S_{x x}(f)},
$$

where $S_{x x}(f)$ is the auto spectrum of changes in arterial blood pressure and $S_{x y}(f)$ is the cross-spectrum between the ABP- and CBFV-signals. For calculating crossand autospectra also the power spectral densities of ABP and CBFV were calculated. The transfer function magnitude $|H(f)|$ and phase spectrum $\Phi(f)$ were derived from the real part $H_{R}(f)$ and imaginary part $H_{I}(f)$ of the complex transfer function as

$$
\begin{aligned}
& |H(f)|=\sqrt{\left[H_{R}(f)\right]^{2}+\left[H_{I}(f)\right]^{2}} \\
& \Phi(f)=\arctan \frac{H_{I}(f)}{H_{R}(f)} .
\end{aligned}
$$

The squared coherence function $\gamma^{2}(f)$ was estimated by

$$
\gamma^{2}(f)=\frac{\left|S_{x y}(f)\right|^{2}}{S_{x x}(f) S_{y y}(f)},
$$

where $S_{y y}(f)$ is the auto spectrum of changes in cerebral blood flow velocity. The squared coherence reflects the strength of the linear relationship between ABP and $\mathrm{CBFV}$ for each frequency on a scale from 0 to 1 . Gain and phase were evaluated at $0.1 \mathrm{~Hz}$, because this equals the frequency induced by varying pump flow. The gain is the magnitude of the transfer function between CBFV and ABP and is 
expressed as \% change per mmHg. The phase lag between CBFV and ABP as defined by phase angle shift is regarded to be the dominant and most relevant parameter to quantify dCA [29]. Theoretically, phase may range from $-\pi$ to $+\pi$ radians. In awake human in supine resting position, a positive phase angle around $0.9 \pm 0.3$ indicates normal autoregulation [15]. From the estimated transfer function a step response can be calculated. According to the method introduced by Tiecks et al [50] dCA can also be evaluated by assessing the autoregulation index (ARI). ARI is calculated by fitting the first 5 seconds of the step response function resulting from the transfer function analysis in the same way as described previously [15].

By changing $\mathrm{p}_{\mathrm{a}} \mathrm{CO}_{2}$ and measuring the mean $\mathrm{CBFV}$ at each level of $\mathrm{p}_{\mathrm{a}} \mathrm{CO}_{2}$, cerebral carbon dioxide reactivity can be evaluated. $\mathrm{CO}_{2} \mathrm{R}$ is expressed as the percent change in $\mathrm{SctO}_{2}$, and blood flow velocity in the middle cerebral artery per $\mathrm{mmHg}$ change in $\mathrm{p}_{\mathrm{a}} \mathrm{CO}_{2}$. The mean values of five minute recording of $\mathrm{SctO}_{2} / \mathrm{CBFV}$ at each $\mathrm{p}_{\mathrm{a}} \mathrm{CO}_{2}$ level are used to calculate both absolute and percentage change in $\mathrm{CBFV}$ and $\mathrm{SctO}_{2}$ using the formulae proposed by Kadoi et al [21].

$$
\begin{aligned}
& \mathrm{CO}_{2} R_{\text {NIRS,hypo }}=\frac{\operatorname{SctO}_{2}(40)-\mathrm{SctO}_{2}(30)}{(40-30) \cdot \operatorname{SctO}_{2}(40)} \cdot 100 \% \\
& \mathrm{CO}_{2} R_{\text {NIRS,hyper }}=\frac{\operatorname{SctO}_{2}(50)-\operatorname{SctO}_{2}(40)}{(50-40) \cdot \operatorname{SctO}_{2}(40)} \cdot 100 \% \\
& \mathrm{CO}_{2} R_{T C D, \text { hypo }}=\frac{C B F V(40)-C B F V(30)}{(40-30) \cdot C B F V(40)} \cdot 100 \% \\
& C O_{2} R_{T C D, \text { hyper }}=\frac{C B F V(50)-C B F V(40)}{(50-40) \cdot C B F V(40)} \cdot 100 \%
\end{aligned}
$$

where $\mathrm{SctO}_{2}$ and CBFV are the values measured for the specified level of $\mathrm{p}_{\mathrm{a}} \mathrm{CO}_{2}$.

\section{Statistics}

Statistic analyses were performed using the Statistical Package for Social Sciences (SPSS) version 16.0 for Windows. Data normality was tested using the Kolgomorov-Smirnov test. If normally distributed, the paired Student's t-test with Bonferroni correction was used to compare results between different levels of $\mathrm{p}_{\mathrm{a}} \mathrm{CO}_{2}$ otherwise the Wilcoxon signed-rank test was used. 
Chapter 6

Table 2 Cerebral autoregulation parameters during CPB; mean (std)

\begin{tabular}{|c|c|c|c|c|}
\hline $\mathrm{p}_{\mathrm{a}} \mathrm{CO}_{2}$ & $\begin{array}{c}30 \mathrm{mmHg} \\
\mathrm{n}=36\end{array}$ & $\begin{array}{c}40 \mathrm{mmHg} \\
\mathrm{n}=37\end{array}$ & $\begin{array}{c}50 \mathrm{mmHg} \\
\mathrm{n}=37\end{array}$ & \\
\hline Mean ABP $[\mathrm{mmHg}]$ & $76(11)$ & 80 (11) & $80(10)$ & \\
\hline Std ABP $[\mathrm{mmHg}]$ & $7.4(3)$ & $7.0(3)$ & $7.4(3)$ & \\
\hline $\begin{array}{l}\text { PSD ABP at } 0.1 \mathrm{~Hz} \\
{\left[\mathrm{mmHg}^{2} / \mathrm{Hz}\right] * 1000}\end{array}$ & $0.58(0.4)$ & $0.65(0.4)$ & $0.81(0.5)$ & $b^{*} c^{* *}$ \\
\hline Mean CBFV left $[\mathrm{cm} / \mathrm{s}]$ & $25(11)$ & $33(12)$ & $48(17)$ & $a^{* *} b^{* *} c^{* *}$ \\
\hline Std CBFV left $[\mathrm{cm} / \mathrm{s}]$ & $3.6(1)$ & $3.9(1)$ & $5.2(3)$ & $b^{* *} c^{*}$ \\
\hline $\begin{array}{l}\text { PSD CBFV left at } 0.1 \mathrm{~Hz} \\
{\left[(\mathrm{~cm} / \mathrm{s})^{2} / \mathrm{Hz}\right]^{*} 1000}\end{array}$ & $0.33(0.2)$ & $0.30(0.2)$ & $0.33(0.3)$ & \\
\hline Mean CBFV right $[\mathrm{cm} / \mathrm{s}]$ & $24(8)$ & $32(11)$ & $46(16)$ & $a^{* *} b^{* *} c^{* *}$ \\
\hline Std CBFV right $[\mathrm{cm} / \mathrm{s}]$ & $3.4(1)$ & $3.8(2)$ & $4.8(2)$ & $b^{* *} c^{*}$ \\
\hline $\begin{array}{l}\text { PSD CBFV right @ } 0.1 \mathrm{~Hz} \\
{\left[(\mathrm{~cm} / \mathrm{s})^{2} / \mathrm{Hz}\right]{ }^{*} 1000}\end{array}$ & $0.28(0.2)$ & $0.30(0.3)$ & $0.36(0.3)$ & \\
\hline Hct $[\%]$ & & $27(4)$ & & \\
\hline $\begin{array}{l}\mathrm{CO}_{2} \text { Reactivity TCD } \\
{[\% / \mathrm{mmHg}]}\end{array}$ & $2.5(1)$ & & $5.0(2)$ & $b^{* *}$ \\
\hline SctO2 NIRS [\%] & $65(4)$ & $66(4)$ & $69(3)$ & $a^{* *} b^{* *} c^{* *}$ \\
\hline $\begin{array}{l}\mathrm{CO}_{2} \text { Reactivity NIRS } \\
{[\% / \mathrm{mmHg}]}\end{array}$ & $0.25(0.3)$ & & $0.45(0.3)$ & $b^{*}$ \\
\hline Coherence at $0.1 \mathrm{~Hz}$ & $0.90(0.1)$ & $0.91(0.09)$ & $0.95(0.06)$ & $b^{*} c^{*}$ \\
\hline Gain at $0.1 \mathrm{~Hz}[\% / \mathrm{mmHg}]$ & $3.4(2)$ & $2.2(0.9)$ & $1.4(0.4)$ & $a^{* *} b^{* *} c^{* *}$ \\
\hline Phase at $0.1 \mathrm{~Hz}[\mathrm{rad}]$ & $0.58(0.3)$ & $0.31(0.2)$ & $0.10(0.1)$ & $a^{* *} b^{* *} c^{* *}$ \\
\hline ARI & $5.8(1)$ & $4.8(1)$ & $3.0(2)$ & $a^{* *} b^{* *} c^{* *}$ \\
\hline
\end{tabular}

a (30 vs. $40 \mathrm{mmHg}), \mathrm{b}(30$ vs. $50 \mathrm{mmHg})$, c (40 vs. $50 \mathrm{mmHg}),{ }^{*} \mathrm{p}<0.05^{* *} \mathrm{p}<0.01$ after Bonferroni correction 

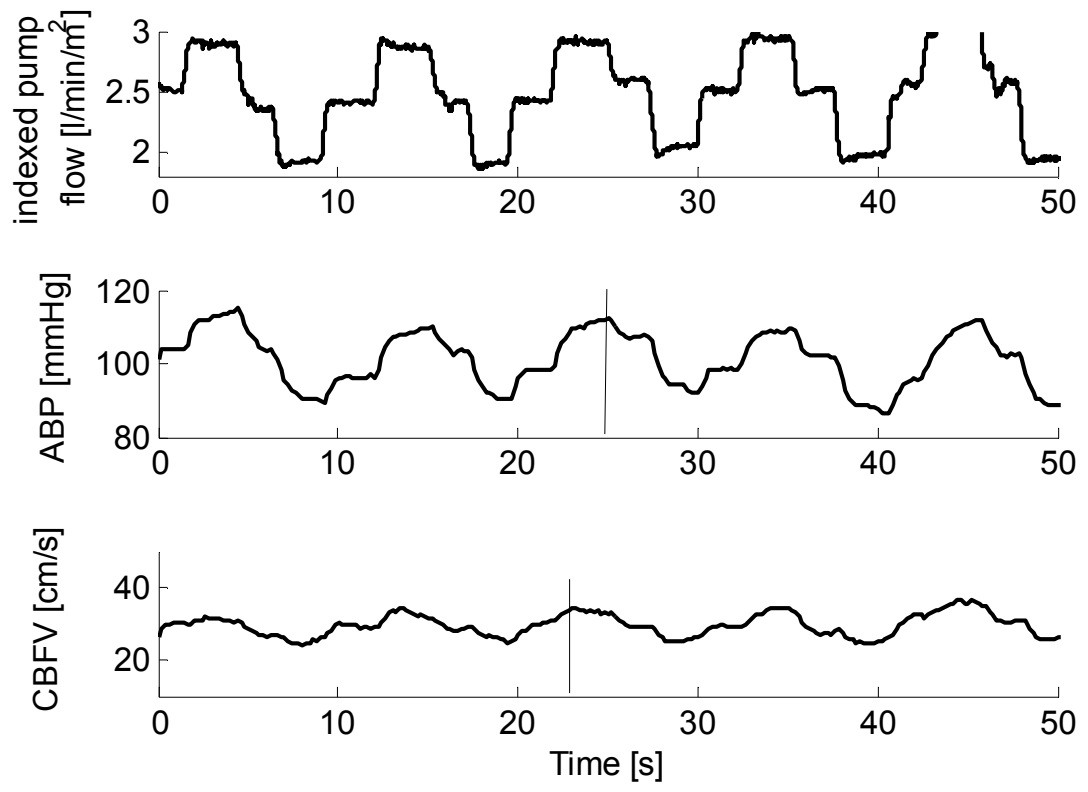

Figure 1 Effect of cyclic changing pump flow (upper panel) on arterial blood pressure (ABP, middle panel) and cerebral blood flow velocity (CBFV) in the middle cerebral arteries (lower panel) at $\mathrm{p}_{\mathrm{a}} \mathrm{CO}_{2}=40 \mathrm{mmHg}$ in a patient undergoing $\mathrm{CPB}$.

\section{Results}

Because of technical problems the measurement at $\mathrm{p}_{\mathrm{a}} \mathrm{CO}_{2}=30 \mathrm{mmHg}$ could not be performed in a single patient. All other measurements were recorded without problems. Figure 1 shows an example of the signals recorded during the induced changes in pump flow at $\mathrm{p}_{\mathrm{a}} \mathrm{CO}_{2}=40 \mathrm{mmHg}$ clearly showing the stepwise increase and decrease of indexed pump flow. ABP and CBFV vary with the same 6/min frequency. Based on these ABP and CBFV wave-shaped signals dCA was evaluated using transfer function analysis.

Table 2 shows group results for three $\mathrm{p}_{\mathrm{a}} \mathrm{CO}_{2}$ levels. Mean $\mathrm{ABP}$ and its standard deviation are not significantly different for $\mathrm{p}_{\mathrm{a}} \mathrm{CO}_{2}=30 \mathrm{mmHg}$ (hypocapnia) compared to 40 (normocapnia) and $50 \mathrm{mmHg}$ (hypercapnia), whereas power spectral density at $0.1 \mathrm{~Hz}$ is significantly higher for hypercapnia compared to both hypocapnia $(\mathrm{p}<0.05)$ and normocapnia $(\mathrm{p}<0.01)$. Figure 2 shows power spectral density for ABP and CBFV. The ABP peak at $0.1 \mathrm{~Hz}$ increases with $\mathrm{p}_{\mathrm{a}} \mathrm{CO}_{2}$ which is not true for the CBFV peak. 

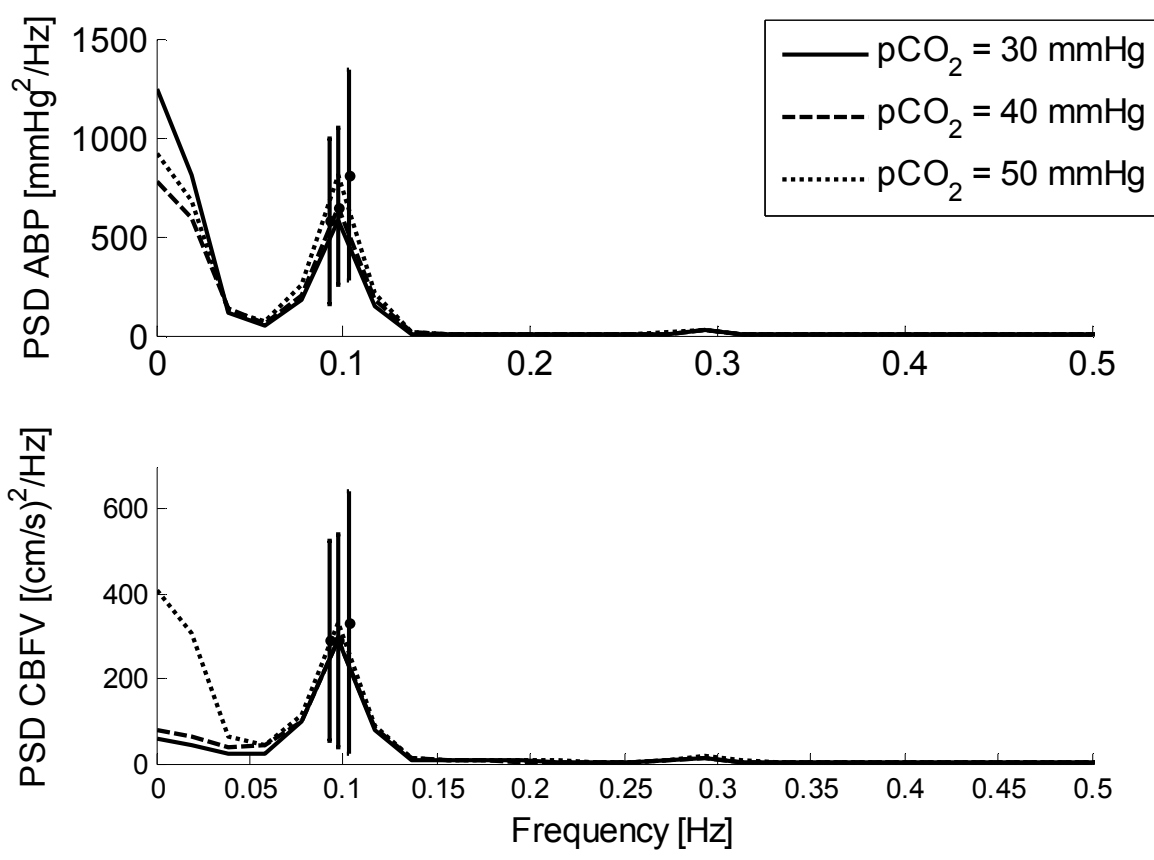

Figure 2 Group averaged power spectral densities of ABP and CBFV during cardiopulmonary bypass at three different $\mathrm{p}_{\mathrm{a}} \mathrm{CO}_{2}$ levels. Error bars represent \pm 1 standard deviation at $0.1 \mathrm{~Hz}$.

Since no significant differences are found between left and right MCA CBFV only left-right averaged results are presented for $\mathrm{CO}_{2} \mathrm{R}$ and dCA. In the left and right MCA mean CBFV is significantly lower $(\mathrm{p}<0.01)$ for hypocapnia compared to both normocapnia and hypercapnia. Although ABP standard deviation was equal for all three $\mathrm{p}_{\mathrm{a}} \mathrm{CO}_{2}$ levels, $\mathrm{CBFV}$ standard deviation is significantly lower for hypocapnia compared to both normocapnia $(\mathrm{p}<0.05)$ and hypercapnia $(\mathrm{p}<0.01)$. No significant differences were found for CBFV power spectral density at $0.1 \mathrm{~Hz}$.

Figure 3 shows group averaged dCA plots. Coherence is significantly higher $(\mathrm{p}<0.05)$ for hypercapnia compared to both hypocapnia and normocapnia. dCA parameters gain, phase and ARI are significantly higher $(\mathrm{p}<0.01)$ for hypocapnia compared to both normo- and hypercapnia. Moreover, normocapnia values are significantly lower $(\mathrm{p}<0.01)$ than hypercapnia values. Figure 4 shows group averaged dCA step responses for the three $\mathrm{p}_{\mathrm{a}} \mathrm{CO}_{2}$ levels. The initial step response rise decreases from hypocapnia to normocapnia and hypercapnia whereas the end level is highest for hypercapnia indicating impaired autoregulation. 
TCD based $\mathrm{CO}_{2} \mathrm{R}$ is significantly higher $(\mathrm{p}<0.01)$ for the hypercapnic change (5.0 $\pm 2 \% / \mathrm{mmHg})$ compared to the hypocapnic change $(2.5 \pm 1 \% / \mathrm{mmHg})$.
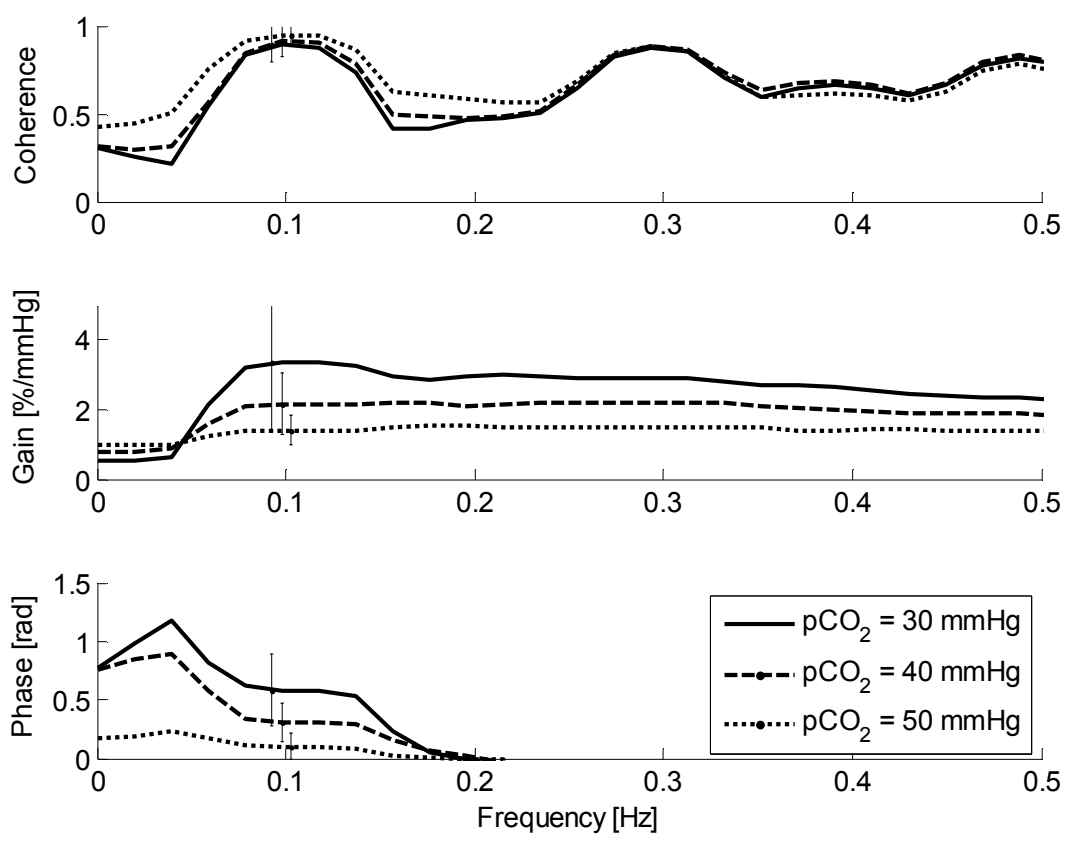

Figure 3 Group averaged transfer function analysis showing averaged coherence (upper panel), gain (middle panel) and phase (lower panel) during cardiopulmonary bypass at three different $\mathrm{p}_{\mathrm{a}} \mathrm{CO}_{2}$ levels. Error bars represent \pm 1 standard deviation at $0.1 \mathrm{~Hz}$.

Figure 5 shows NIRS $\mathrm{CO}_{2} \mathrm{R}$ measurements for a typical patient for the three conditions. It shows that cerebral oxygenation increases with the $\mathrm{p}_{\mathrm{a}} \mathrm{CO}_{2}$ level. Similarly to TCD based $\mathrm{CO}_{2} \mathrm{R}$, NIRS based $\mathrm{CO}_{2} \mathrm{R}$ is calculated and is also significantly higher $(\mathrm{p}<0.05)$ for the hypercapnic change $(0.45 \pm 0.3 \% / \mathrm{mmHg})$ compared to the hypocapnic change $(0.25 \pm 0.3 \% / \mathrm{mmHg})$.

\section{Discussion}

In this study we demonstrated the feasibility to induce arterial blood pressure variations for $\mathrm{dCA}$ evaluation by changing $\mathrm{CPB}$ pump flow at 6 cycles/min. The induced stepwise changes in pump flow by repetitively switching between three levels of indexed pump flow resulted in a staircase shaped pump flow pattern. 
Due to compliance of the arterial vascular bed a more smoothed almost sinusoidal variation is induced in arterial blood pressure. The resulting cerebral blood flow velocity shows a similar sinusoidal shape. The resemblance of $A B P$ and CBFV signals to "pure" sine waves is confirmed by their power spectral densities which show a clear peak at $0.1 \mathrm{~Hz}$ and an almost hundredfold lower peak at the third harmonic at $0.3 \mathrm{~Hz}$. The width of the peak at $0.1 \mathrm{~Hz}$ is partly due to the three-point triangular spectral smoothing that was applied [17]. Since the evoked flow changes are square wave shaped only odd harmonics $\left(3^{\text {rd }}, 5^{\text {th }}\right.$, etc. $)$ are expected. For transfer function analysis of dCA the induced variations are well suited, as shown in figure 3.

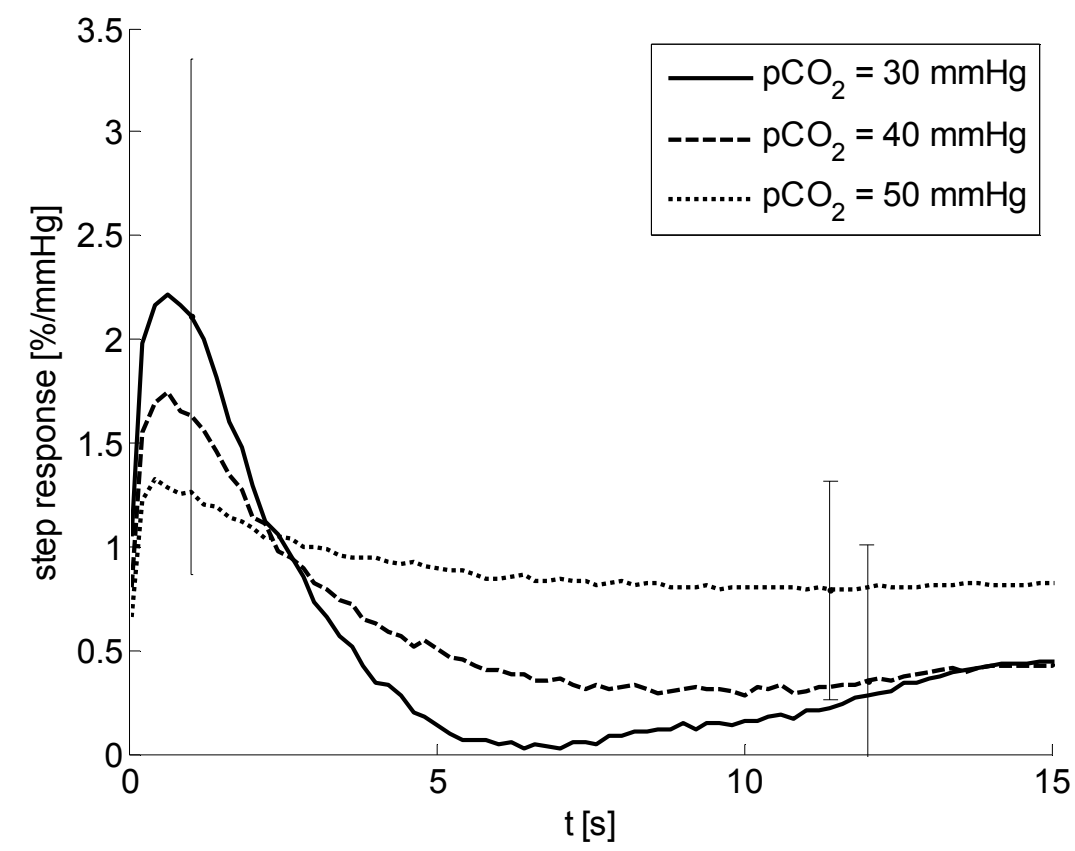

Figure 4 Group averaged step responses during cardiopulmonary bypass at three different $\mathrm{p}_{\mathrm{a}} \mathrm{CO}_{2}$ levels. Error bars represent the largest \pm 1 standard deviation.

To evaluate dCA during CPB sufficient blood pressure variability combined with coherent CBFV variability is necessary for reliable transfer function estimation. Little is known about spontaneous ABP variability during CPB. Nicolet et al [26] hypothesized that in absence of cardio respiratory variability during $\mathrm{CPB}$ extreme situations in physiologic control of CBF should be revealed. During extracorporeal circulation they found no significant coherence between $A B P$ and $\mathrm{CBFV}$, possibly due to different dominant frequencies occurring in $\mathrm{ABP}(9 \mathrm{mHz})$ 
and CBFV $(70 \mathrm{mHz})$. The authors hypothesized CPB provokes a highly protective mechanism, thereby reducing $\mathrm{CBF}$ fluctuations by deactivation of $\mathrm{B}$ waves, revealing monotonous $\mathrm{UB}$ waves. The $0.1 \mathrm{~Hz}$ frequency we induced showed very high $(\geq 0.9)$ coherence between $\mathrm{ABP}$ and $\mathrm{CBFV}$, reflecting a strong linear relation between variability in $\mathrm{ABP}$ and $\mathrm{CBFV}$. dCA phase, which decreased from hypocapnia to hypercapnia, showed to be independent on coherence since coherence was already high for each level of $\mathrm{p}_{\mathrm{a}} \mathrm{CO}_{2}$.

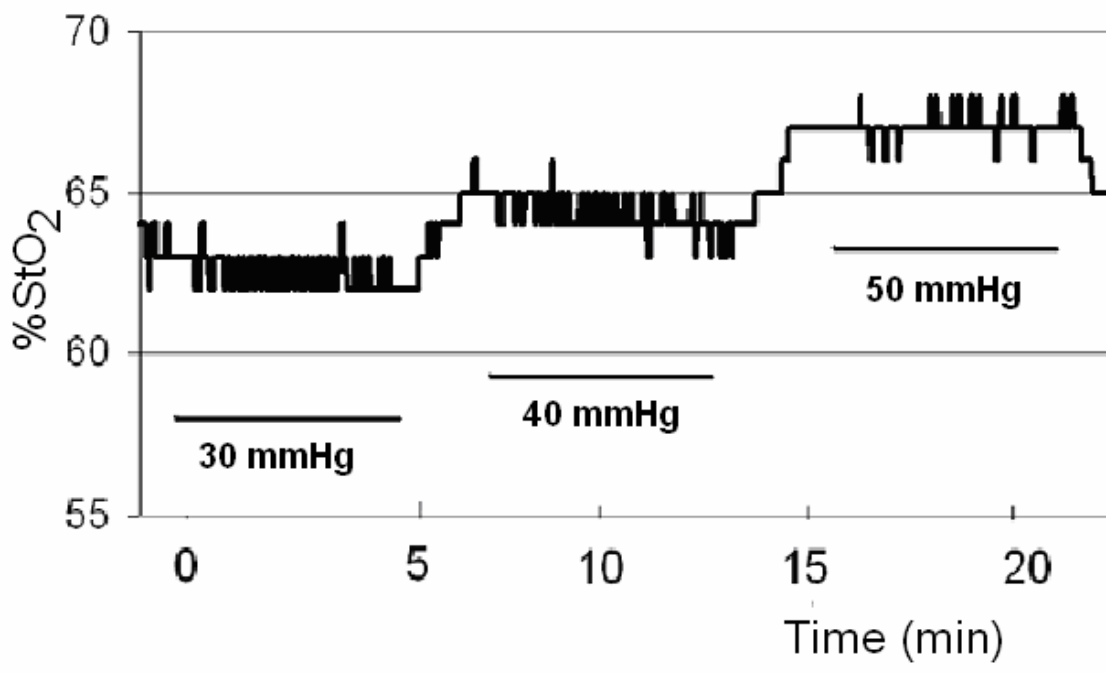

Figure 5 Example measurement of five minute episodes of cerebral carbon dioxide reactivity testing by near-infrared spectroscopy $\left(\mathrm{SctO}_{2}\right)$ at hypocapnia $(30 \mathrm{mmHg})$, normocapnia $(40 \mathrm{mmHg})$ and hypercapnia $(50 \mathrm{mmHg})$.

The sinusoidal varying $\mathrm{ABP}$ and $\mathrm{CBFV}$ signals also allow visual appreciation of phase shift between $A B P$ and $C B F V$. Figure 1 shows a positive phase shift since $\mathrm{CBFV}$ waves are occurring earlier than $\mathrm{ABP}$ waves as indicated by the vertical lines. The actual value of this phase shift is hard to estimate due to the variation in individual wave shapes, but it can be reliably estimated through transfer function analysis. This is calculated offline, but can be implemented online as well. Group averaged results show a phase shift at $0.1 \mathrm{~Hz}$ of approximately 0.3 rad in normocapnia (figure 2) which is markedly reduced compared to phase angles of 0.9 rad under normal circumstances $[15,16]$. Reduced phase during CPB may partly be due to the effect of anaesthesia [12] although two studies showed that anaesthesia through propofol preserved cerebral autoregulation [38, 49]. Propofol decreases systemic vascular resistance which can be troublesome in 


\section{Chapter 6}

hypovolemic bleeding patients and cerebral autoregulation can be compromised [18] which is improbable in patients undergoing CPB. Ogawa et al [27] showed impaired dCA due to hypervolemic hemodilution with increased gain after infusion of $15-30 \mathrm{ml} / \mathrm{kg}$ of saline with a mean Hct of $43 \%$. Also phase decreased, but this did not reach statistical significance. Hemodilution during CPB therefore seems to be a plausible explanation for the reduced dCA capability, since Hct with $27 \%$ is much lower in our study group.

Because the induced sinusoidal variations at $0.1 \mathrm{~Hz}$ are the main content of $A B P$ and CBFV signals and their shapes are very similar, coherence is high, even close to 1, expressing the strong linear relation between ABP and CBFV. Because the shape is not a purely sinusoidal, also at higher harmonics $(0.3 \mathrm{~Hz}$ and $0.5 \mathrm{~Hz})$ coherence is high. These high coherence values are unprecedented in beating heart measurement and indicate a strong linear relationship between ABP and CBFV under the conditions of this study. It also shows that in univariate analysis all variance of $C B F V$ can be explained by ABP variance and coherence can reach values of nearly 1 when signal to noise ratio is high. However, in beating heart measurements, these signal to noise ratios are not easily achieved possibly due to others sources of $\mathrm{CBFV}$ variations that are not related to $\mathrm{ABP}$ variations, such as the influence of changing $\mathrm{p}_{\mathrm{a}} \mathrm{CO}_{2}$ and/or sympathetic tone as was shown by others studying multiple coherence $[33,37]$.

Gain decreases with increasing $\mathrm{p}_{\mathrm{a}} \mathrm{CO}_{2}$, not only at $0.1 \mathrm{~Hz}$, but also at higher 0.3 and $0.5 \mathrm{~Hz}$ frequencies at which dCA is thought not to function. In an earlier study in awake lacunar infarct patients we found decreased gain after acetazolamide infusion [17]. Since acetazolamide is an anhydrase inhibitor it has the same effect as hypercapnia. Others did not report dCA gain [5] or report no reduction [4] or even an increase [53] at hypercapnia. However, they all investigated much smaller number (10-14) of subjects. An explanation for our finding is that decreased gain with increased $\mathrm{p}_{\mathrm{a}} \mathrm{CO}_{2}$ might be due to increased vessel dilation causing less responsiveness to changes in pressure. Given the different results for dCA gain it seems not to be a very robust parameter of dCA as Panerai demonstrated using simulations with the Tiecks model [29].

The reduction of the initial rise of the step response function, which is clearly shown in figure 4, may be explained by a decrease in critical closing pressure. With increasing $\mathrm{p}_{\mathrm{a}} \mathrm{CO}_{2}$ a decrease of critical closing pressure occurs as was shown by others $[4,32]$. Since we investigated patients during CPB we cannot evaluate critical closing pressure using the available per beat techniques $[2,30$, 
34]. Regression analysis of the $0.1 \mathrm{~Hz}$ waves in our data to determine zero-flow pressure could be performed, but its relation with critical closing pressure is unclear.

For phase angle the impairment of dCA with increasing $\mathrm{p}_{\mathrm{a}} \mathrm{CO}_{2}$ is much clearer. At $0.1 \mathrm{~Hz}$, phase angle clearly decreases with increasing $\mathrm{p}_{\mathrm{a}} \mathrm{CO}_{2}$, whereas at 0.3 and $0.5 \mathrm{~Hz}$, phase angle is close to zero for all levels of $\mathrm{p}_{\mathrm{a}} \mathrm{CO}_{2}$, indicating that $\mathrm{dCA}$ is not functioning at these higher frequencies. Also, the decrease in ARI with $\mathrm{p}_{\mathrm{a}} \mathrm{CO}_{2}$ confirms decreased dCA functioning. Impairment of dCA with hypercapnia was also shown by others using a thigh cuff deflation method as well as by transfer function analysis $[3,4,22]$.

dCA results, especially high coherence and worsened autoregulation at increasing levels of $\mathrm{p}_{\mathrm{a}} \mathrm{CO}_{2}$, demonstrate that our technique is feasible and permits dCA quantification during $\mathrm{CPB}$. In this way testing of dCA may facilitate e.g. studies on the effect of different perfusion strategies or the effect of impaired dCA on post surgery stroke incidence. Recently, Ono et al [28] showed that impaired cerebral autoregulation during $\mathrm{CPB}$, as evaluated by $\mathrm{Mx}$, is associated with increased postoperative stroke risk. Their Mx measure for cerebral autoregulation is difficult to compare with dCA transfer function analysis parameters, since it concerns different frequencies. We analysed the ABP-CBFV relation at the induced $0.1 \mathrm{~Hz}$ frequency, whereas for Mx with a 10 second time resolution 30 consecutive points were used to calculate a cross correlation between ABP and $\mathrm{CBFV}$ or $\mathrm{SctO}_{2}$ and so, for $\mathrm{Mx}$ much lower frequencies $(\mathrm{f}<0.05 \mathrm{~Hz})$ are considered.

The effect of $\mathrm{p}_{\mathrm{a}} \mathrm{CO}_{2}$ on $\mathrm{CBFV}$ and $\mathrm{SctO}_{2}$ shows intact $\mathrm{CO}_{2} \mathrm{R}$ in the range between 30 and $50 \mathrm{mmHg}$ during $\mathrm{CPB}$ although there is a clear $\mathrm{CO}_{2} \mathrm{R}$ asymmetry for hypocapnia compared to hypercapnia. Other investigators showed an exponential relationship between end tidal $\mathrm{CO}_{2}$ and $\mathrm{CBFV}[20,23]$. Since we controlled for arterial $\mathrm{CO}_{2}$ which is strongly correlated to end tidal $\mathrm{CO}_{2}[8,36]$ the relationship between arterial $\mathrm{CO}_{2}$ and $\mathrm{CBFV}$ can also be considered to be exponential. This explains why for hypocapnia $\mathrm{CO}_{2} \mathrm{R}$ is lower compared to $\mathrm{CO}_{2} \mathrm{R}$ for hypercapnia.

\section{Limitations}

With TCD due to unknown blood vessel diameter no absolute flow but blood flow velocity is measured. Huber et al [19] showed via angiography that cerebral arteries with a diameter of at least $2.5 \mathrm{~mm}$ do not show changes in diameter with 
hypercapnia, hyperventilation, hypertonic glucose and administration of papaverine. Therefore the assumption of constant vessel diameter seems legitimate for this study.

\section{Conclusion}

Studying $\mathrm{dCA}$ and $\mathrm{CO}_{2} \mathrm{R}$ during $\mathrm{CPB}$ is feasible by changing pump flow at a $6 / \mathrm{min}$ rate. Induced blood pressure variations result in high coherence for transfer function analysis, indicating that $\mathrm{ABP}$ and $\mathrm{CBFV}$ are strongly linearly related. Moreover, increased arterial $\mathrm{CO}_{2}$ levels clearly lead to impaired cerebral autoregulation. Future studies that use TCD and NIRS to evaluate $\mathrm{dCA}$ and $\mathrm{CO}_{2} \mathrm{R}$ will hopefully clarify optimal perfusion strategies for various patient populations scheduled for operations involving CPB.

\section{Acknowledgements}

The authors thank Prof. R.B. Panerai (University of Leicester, UK) for his valuable contributions to the manuscript.

\section{References}

1. Aaslid R. Cerebral autoregulation and vasomotor reactivity. Front Neurol Neurosci 21: 216-228, 2006.

2. Aaslid R, Lash SR, Bardy GH, Gild WH, and Newell DW. Dynamic pressure-flow velocity relationships in the human cerebral circulation. Stroke 34: 1645-1649, 2003.

3. Aaslid R, Lindegaard KF, Sorteberg W, and Nornes H. Cerebral autoregulation dynamics in humans. Stroke 20: 45-52, 1989.

4. Ainslie PN, Celi L, McGrattan K, Peebles K, and Ogoh S. Dynamic cerebral autoregulation and baroreflex sensitivity during modest and severe step changes in arterial PCO2. Brain Res 1230: 115-124, 2008.

5. Birch AA, Dirnhuber MJ, Hartley-Davies R, Iannotti F, and Neil-Dwyer G. Assessment of autoregulation by means of periodic changes in blood pressure. Stroke 26: 834-837, 1995.

6. Bishop CC, Powell S, Rutt D, and Browse NL. Transcranial Doppler measurement of middle cerebral artery blood flow velocity: a validation study. Stroke 17: 913-915, 1986.

7. Brady K, Joshi B, Zweifel C, Smielewski P, Czosnyka M, Easley RB, and Hogue CW, Jr. Real-Time Continuous Monitoring of Cerebral Blood 
Flow Autoregulation Using Near-Infrared Spectroscopy in Patients Undergoing Cardiopulmonary Bypass. Stroke 41: 1951-1956, 2010.

8. Brothers RM, Ganio MS, Hubing KA, Hastings JL, and Crandall CG. End-tidal carbon dioxide tension reflects arterial carbon dioxide tension in the heat-stressed human with and without simulated hemorrhage. American Journal of Physiology - Regulatory, Integrative and Comparative Physiology 300: R978-R983, 2011.

9. Brugniaux JV, Hodges AN, Hanly PJ, and Poulin MJ. Cerebrovascular responses to altitude. Respir Physiol Neurobiol 158: 212-223, 2007.

10. Caplan LR, and Hennerici M. Impaired clearance of emboli (washout) is an important link between hypoperfusion, embolism, and ischemic stroke. Arch Neurol 55: 1475-1482, 1998.

11. Cook DJ, Proper JA, Orszulak TA, Daly RC, and Oliver WC,Jr. Effect of pump flow rate on cerebral blood flow during hypothermic cardiopulmonary bypass in adults. J Cardiothorac Vasc Anesth 11: 415-419, 1997.

12. Dagal A, and Lam AM. Cerebral autoregulation and anesthesia. Curr Opin Anaesthesiol 22: 547-552, 2009.

13. Diehl RR, Linden D, Lucke D, and Berlit P. Phase relationship between cerebral blood flow velocity and blood pressure. A clinical test of autoregulation. Stroke 26: 1801-1804, 1995.

14. Diehl RR, Linden D, Lucke D, and Berlit P. Spontaneous blood pressure oscillations and cerebral autoregulation. Clin Auton Res 8: 7-12, 1998.

15. Gommer E, Shijaku E, Mess W, and Reulen J. Dynamic cerebral autoregulation: different signal processing methods without influence on results and reproducibility. Med Biol Eng Comput 1-8, 2010.

16. Gommer ED, Martens EG, Aalten P, Shijaku E, Verhey FR, Mess WH, Ramakers IH, and Reulen JP. Dynamic Cerebral Autoregulation in Subjects with Alzheimer's Disease, Mild Cognitive Impairment, and Controls: Evidence for Increased Peripheral Vascular Resistance with Possible Predictive Value. J Alzheimers Dis 30: 805-813, 2012.

17. Gommer ED, Staals J, van Oostenbrugge RJ, Lodder J, Mess WH, and Reulen JP. Dynamic cerebral autoregulation and cerebrovascular reactivity: a comparative study in lacunar infarct patients. Physiol Meas 29: 1293-1303, 2008.

18. Grathwohl KW, Black IH, Spinella PC, Sweeney J, Robalino J, Helminiak J, Grimes J, Gullick R, and Wade CE. Total intravenous anesthesia including ketamine versus volatile gas anesthesia for combat-related operative traumatic brain injury. Anesthesiology 109: 44-53, 2008. 
19. Huber P, and Handa J. Effect of contrast material, hypercapnia, hyperventilation, hypertonic glucose and papaverine on the diameter of the cerebral arteries. Angiographic determination in man. Invest Radiol 2:1732, 1967.

20. Ide K, Eliasziw M, and Poulin MJ. Relationship between middle cerebral artery blood velocity and end-tidal PCO2 in the hypocapnichypercapnic range in humans. Journal of Applied Physiology 95: 129-137, 2003.

21. Kadoi Y, Saito S, Goto F, and Fujita N. The effect of diabetes on the interrelationship between jugular venous oxygen saturation responsiveness to phenylephrine infusion and cerebrovascular carbon dioxide reactivity. Anesth Analg 99: 325-331, 2004.

22. Low DA, Wingo JE, Keller DM, Davis SL, Cui J, Zhang R, and Crandall CG. Dynamic cerebral autoregulation during passive heat stress in humans. Am J Physiol Regul Integr Comp Physiol 296: R1598-1605, 2009.

23. Markwalder T-M, Grolimund P, Seiler RW, Roth F, and Aaslid R. Dependency of Blood Flow Velocity in the Middle Cerebral Artery on EndTidal Carbon Dioxide Partial Pressure - A Transcranial Ultrasound Doppler Study. J Cereb Blood Flow Metab 4: 368-372, 1984.

24. MurkinJM. The role of CPB management in neurobehavioral outcomes after cardiac surgery. Ann Thorac Surg 59: 1308-1311, 1995.

25. Murkin JM, Martzke JS, Buchan AM, Bentley C, and Wong CJ. A randomized study of the influence of perfusion technique and $\mathrm{pH}$ management strategy in 316 patients undergoing coronary artery bypass surgery. I. Mortality and cardiovascular morbidity.J Thorac Cardiovasc Surg 110: 340-348, 1995.

26. Nicolet J, Gillard T, Gindre G, Cervenansky F, Duale C, Bazin JE, De Riberolles C, Schoeffler P, and Lemaire JJ. Modifications of spontaneous cerebral blood flow oscillations during cardiopulmonary bypass. Acta Neurochir Suppl 95: 337-339, 2005.

27. Ogawa Y, Iwasaki K, Aoki K, Shibata S, Kato J, and Ogawa S. Central hypervolemia with hemodilution impairs dynamic cerebral autoregulation. Anesth Analg 105: 1389-1396, 2007.

28. Ono M, Joshi B, Brady K, Easley RB, Zheng Y, Brown C, Baumgartner $\mathrm{W}$, and Hogue CW. Risks for impaired cerebral autoregulation during cardiopulmonary bypass and postoperative stroke. British Journal of Anaesthesia 109: 391-398, 2012.

29. Panerai RB. Cerebral autoregulation: from models to clinical applications. Cardiovasc Eng 8: 42-59, 2008. 
30. Panerai RB. The critical closing pressure of the cerebral circulation. Med Eng Phys 25: 621-632, 2003.

31. Panerai RB, Dawson SL, and Potter JF. Linear and nonlinear analysis of human dynamic cerebral autoregulation. Am J Physiol 277: H1089-1099, 1999.

32. Panerai RB, Deverson ST, Mahony P, Hayes P, and Evans DH. Effects of $\mathrm{CO} 2$ on dynamic cerebral autoregulation measurement. Physiol Meas 20: 265-275, 1999.

33. Panerai RB, Eames PJ, and Potter JF. Multiple coherence of cerebral blood flow velocity in humans. Am J Physiol Heart Circ Physiol 291: H251H259, 2006.

34. Panerai RB, Salinet ASM, Brodie FG, and Robinson TG. The influence of calculation method on estimates of cerebral critical closing pressure. Physiological Measurement 32: 467-482, 2011.

35. Paulson OB, Strandgaard S, and Edvinsson L. Cerebral autoregulation. Cerebrovasc Brain Metab Rev 2: 161-192, 1990.

36. Peebles K, Celi L, McGrattan K, Murrell C, Thomas K, and Ainslie PN. Human cerebrovascular and ventilatory $\mathrm{CO} 2$ reactivity to end-tidal, arterial and internal jugular vein PCO2.J Physiol 584: 347-357, 2007.

37. Peng T, Rowley A, Ainslie P, Poulin M, and Payne S. Multivariate System Identification for Cerebral Autoregulation. Annals of Biomedical Engineering 36: 308-320, 2008.

38. Petersen KD, Landsfeldt U, Cold GE, Petersen CB, Mau S, Hauerberg J, Holst $\mathrm{P}$, and Olsen KS. Intracranial pressure and cerebral hemodynamic in patients with cerebral tumors: a randomized prospective study of patients subjected to craniotomy in propofol-fentanyl, isoflurane-fentanyl, or sevoflurane-fentanyl anesthesia. Anesthesiology 98: 329-336, 2003.

39. Rasmussen P, Dawson EA, Nybo L, van Lieshout JJ, Secher NH, and Gjedde A. Capillary-oxygenation-level-dependent near-infrared spectrometry in frontal lobe of humans. J Cereb Blood Flow Metab 27: 10821093, 2007.

40. Roach GW, Kanchuger M, Mangano CM, Newman M, Nussmeier N, Wolman R, Aggarwal A, Marschall K, Graham SH, Ley C, Ozanne G, Mangano DT, Herskowitz A, Katseva V, and Sears R. Adverse Cerebral Outcomes after Coronary Bypass Surgery. New England Journal of Medicine 335: 1857-1864, 1996.

41. Rosamond W, Flegal K, Friday G, Furie K, Go A, Greenlund K, Haase N, Ho M, Howard V, Kissela B, Kittner S, Lloyd-Jones D, McDermott M, Meigs J, Moy C, Nichol G, O'Donnell CJ, Roger V, Rumsfeld J, Sorlie P, 
Steinberger J, Thom T, Wasserthiel-Smoller S, and Hong Y. Heart disease and stroke statistics--2007 update: a report from the American Heart Association Statistics Committee and Stroke Statistics Subcommittee. Circulation 115: e69-171, 2007.

42. Rudolph JL, Sorond FA, Pochay VE, Haime M, Treanor P, Crittenden MD, and Babikian VL. Cerebral Hemodynamics during Coronary Artery Bypass Graft Surgery: The Effect of Carotid Stenosis. Ultrasound in Medicine er Biology 35: 1235-1241, 2009.

43. Sadahiro M, Haneda K, and Mohri H. Experimental study of cerebral autoregulation during cardiopulmonary bypass with or without pulsatile perfusion. J Thorac Cardiovasc Surg 108: 446-454, 1994.

44. Schell RM, Kern FH, Greeley WJ, Schulman SR, Frasco PE, Croughwell ND, Newman M, and Reves JG. Cerebral blood flow and metabolism during cardiopulmonary bypass. Anesth Analg 76: 849-865, 1993.

45. Schmidt M, Scheunert T, Steinbach G, Schirmer U, Marx T, Freitag N, and Reinelt $\mathrm{H}$. Hypertension as a risk factor for cerebral injury during cardiopulmonary bypass. Protein S100B and transcranial Doppler findings. Anaesthesia 56: 733-738, 2001.

46. Schwartz AE, Sandhu AA, Kaplon RJ, Young WL, Jonassen AE, Adams DC, Edwards NM, Sistino JJ, Kwiatkowski P, and Michler RE. Cerebral blood flow is determined by arterial pressure and not cardiopulmonary bypass flow rate. Ann Thorac Surg 60:165-169; discussion 169-170, 1995.

47. Smielewski P, Kirkpatrick P, Minhas P, Pickard JD, and Czosnyka M. Can cerebrovascular reactivity be measured with near-infrared spectroscopy? Stroke 26: 2285-2292, 1995.

48. Sorteberg W, Lindegaard KF, Rootwelt K, Dahl A, Nyberg-Hansen R, Russell D, and Nornes H. Effect of acetazolamide on cerebral artery blood velocity and regional cerebral blood flow in normal subjects. Acta Neurochir (Wien) 97: 139-145, 1989.

49. Strebel S, Lam AM, Matta B, Mayberg TS, Aaslid R, and Newell DW. Dynamic and static cerebral autoregulation during isoflurane, desflurane, and propofol anesthesia. Anesthesiology 83: 66-76, 1995.

50. Tiecks FP, Lam AM, Aaslid R, and Newell DW. Comparison of static and dynamic cerebral autoregulation measurements. Stroke 26: 10141019, 1995.

51. Totaro R, Marini C, Baldassarre M, and Carolei A. Cerebrovascular reactivity evaluated by transcranial Doppler: reproducibility of different methods. Cerebrovasc Dis 9: 142-145, 1999. 
Assessment of dCA during CPB

52. van Mook WNKA, Rennenberg RJMW, Schurink GW, van Oostenbrugge RJ, Mess WH, Hofman PAM, and de Leeuw PW. Cerebral hyperperfusion syndrome. The Lancet Neurology 4: 877-888, 2005.

53. Zhang R, Zuckerman JH, Giller CA, and Levine BD. Transfer function analysis of dynamic cerebral autoregulation in humans. Am J Physiol 274: H233-241, 1998. 

Chapter 7

General Discussion 


\section{General Discussion}

Cerebral autoregulation is a homeostatic mechanism to compensate cerebral blood flow (CBF) for changes in cerebral perfusion pressure (CPP) [3, 27]. Much research in the field of cerebral autoregulation has been aimed at noninvasively investigating this mechanism, eventually leading to a diagnostic tool. Using TCD, CBFV can be determined as a noninvasive measure for CBF assuming the diameter of the insonated vessel remains constant [2]. CPP is the difference between arterial blood pressure $(\mathrm{ABP})$ and intracranial pressure (ICP). Using finger arterial pressure measurement variations in ABP can be noninvasively measured. Efforts have been made to estimate ICP noninvasively $[13,31,32]$, but since the accuracy is low these methods still have limited value [28]. If intracranial pressure is not expected to be elevated as e.g. in head injury patients, ICP often is assumed to be constant and only variation in $\mathrm{ABP}$ is considered for $\mathrm{dCA}$ evaluation. In this thesis we have mainly been focusing on the univariate transfer function analysis of TCD recorded CBFV variations in relation to $\mathrm{ABP}$ variations. The aim of this thesis was to establish and improve clinical applicability of dynamic cerebral autoregulation evaluation. Our aim has been twofold, a methodological and a clinical one. First, the use of transfer function analysis for quantifying dynamic cerebral autoregulation has been evaluated. The influence of different signal analysis aspects has been studied as well as the influence of different ways of inducing blood pressure variations. Secondly, our aim was to evaluate clinical application of the developed dCA analysis. Dynamic cerebral autoregulation has been studied in several patient groups and the results have been analyzed in relation to disease state.

In this chapter methodological aspects, clinical applications, limitations and future perspectives of dCA evaluation will be discussed. Methodological aspects that will be addressed are blood pressure stimuli for dCA evaluation, transfer function analysis results, reproducibility and multivariate analysis.

\section{Methodological aspects}

\section{Blood pressure variation}

Transfer function analysis between ABP as input and CBFV as output is only reliable with sufficient variation of the input. Spontaneous fluctuations of ABP may occur due to autonomic variability of heart rate resulting in cardiac output changes and eventually blood pressure variation. These spontaneous $A B P$ variations have the advantage not to depend on active patient 
cooperation. Variation in ABP can also be provoked e.g. by respiration $[8,30]$, squat-stand maneuvers $[4,7,40]$ or in- and deflating leg cuffs $[1,14,36]$. With in- and expiration lung volume in- and decreases resulting in de- and increasing stroke volume of the heart, leading to cyclic variation in blood pressure. Asking a subject to perform these respiration maneuvers at a fixed rate results in $\mathrm{ABP}$ variation at the same rate which is also known as respiratory sinus arrhythmia. More powerful oscillations further challenge $\mathrm{dCA}$ and therefore enhance clinical significance of autoregulation tests. Single or repetitive squatting followed by standing (squat-stand) elicits transient changes in central blood volume and peripheral vascular resistance, leading to large changes in arterial pressure and heart rate. Although leading to less powerful blood pressure variations than squat-stand, sit-stand maneuvers may also be used. Van Beek et al [38] showed that this method was well tolerated to study dCA in elderly.

In patients undergoing cardiopulmonary bypass surgery hardly any spontaneous oscillations of blood pressure occur. This since autonomic control cannot influence heart rate when the heart is not beating. So, for studying dCA in these circumstances evoked blood pressure changes are needed. Using cyclic variation of pump flow we could induce variations in $\mathrm{ABP}$ leading to variations in $\mathrm{CBFV}$ (chapter 6). For these evoked ABP oscillations power spectral densities at $0.1 \mathrm{~Hz}$ are higher than for sit-stand maneuvers but lower than for squat-stand maneuvers. The high coherence between $\mathrm{ABP}$ and $\mathrm{CBFV}$ for the oscillations evoked during cardiopulmonary bypass cannot be explained by the high power oscillations alone. Possibly the lack of other sources of variation and so the lack of noise, may have also contributed to this.

Not all studies on dCA evaluation using transfer function analysis report both power spectral density of $\mathrm{ABP}$ and $\mathrm{CBFV}$ and their coherence, but from the available data it seems that coherence increases with magnitude of $\mathrm{ABP}$ variations. A small pilot study (unpublished results) we performed in healthy volunteers seems to confirm this relation, but needs further investigation. Possibly there might also be a relation between the magnitude of ABP variations and $\mathrm{dCA}$ results.

\section{Transfer function analysis}

In chapter 2 different settings for transfer function analysis (TFA) were implemented and dCA results were compared. Transfer function analysis starts with estimating the auto spectra of and cross spectra between ABP and CBFV. However, before estimating the spectra first the offset, respectively low 
frequency trend, of both signal needs to be removed. This was either performed by mean removal or using a smoothness priors detrending method [35]. Spectral estimates can be calculated either through epoch averaging using the Welch method [39] or by spectral smoothing [37]. For both several parameter settings need to be chosen, such as window length, overlap percentage, tapering. Combining detrending and spectral estimate methods four different signal processing combinations have been compared.

For dCA parameters evaluated with the four different combinations in chapter 2 no differences have been found. This could lead to the conclusion that it makes no difference which parameter settings are chosen for TFA. However, this was only investigated with data using either spontaneous blood pressure variations or paced breathing induced blood pressure variations. Furthermore, the four parameter combinations compared are not all possible options. Currently, initiated by the Cerebral Autoregulation Research Network (CARNet, www.car-net.org), a multicenter study (11 participating centers including our hospital) is performed to compare different TFA methods for quantifying dCA. Each center will analyze the same data set using their own standard way of analyzing dCA recordings. Results will be compared between centers to evaluate the influence of different TFA parameter settings. The data set used in this study only consists of recordings using spontaneous blood pressure variations. When dCA is being challenged more by higher amplitude ABP variations e.g. by squat-stand maneuvers possibly other effects of different TFA parameter settings could arise. Hopefully, this study and the cooperation in this CARNet research group will contribute to standardization of TFA in dCA.

In single input-single output TFA, coherence is an indication for the fraction of output variation that can linearly be explained by the input variation. For a perfect linear input-output relation coherence equals one. Coherence can be lower than one for several reasons: 1) if there is noise involved, resulting in input and/or output variation that is not explained by the linear transfer function, 2) if other variation from an additional input contributes to the output; multiple coherence [23] can be used to investigate this, 3) if the transfer function is non-linear; non-linear techniques may be used [12, 15-18, 21].

Giller, who was the first to investigate frequency-dependent behavior of cerebral autoregulation, suggested coherence as a measure of dCA [10]. With intact CA, due to changes in CVR, changes in ABP will be attenuated in CBFV, resulting in low coherence, whereas at the other extreme, if $\mathrm{CA}$ is impaired, CBFV will tend to follow the changes in ABP leading to high values of 
coherence. In chapter 6 we reported high coherence values of nearly one using induced blood pressure changes during $\mathrm{CPB}$. According to Giller's theory these high coherence values would indicate impaired autoregulation, but for different levels of $\mathrm{p}_{\mathrm{a}} \mathrm{CO}_{2}$ different values of phase were found each with high coherence. Coherence itself therefore does not seem to be a good measure for dCA.

To identify if gain or phase is the dominant dCA parameter Panerai [20] simulated the effect of varying ARI on TFA parameters using data generated by the Tiecks-model [36]. By generating data for all ten ARI values ten different transfer functions may be constructed. By combining each of the phase spectra with the gain spectrum of ARI 0 ten step responses can be generated with varying phase and constant gain. In the same way this can be done for an ARI 9 response. Similarly this procedure can be repeated with constant phase and varying gain. The overall conclusion of this simulation was that phase is dominant and that a high gain value should not be accepted as an indication of impaired autoregulation in the presence of higher values of phase.

\section{Reproducibility}

An important property for clinical applicability of a test is reproducibility of its results. When measurements are less reliable or when wide day-to-day variation occurs, it is difficult to identify statistically significant change e.g. between normal and pathological situation. A measure for reproducibility is the intraclass correlation coefficient (ICC). For a widely used physiological parameter as for example systolic blood pressure measurement using an automated blood pressure unit ICC values above 0.8 have been found [34] which is considered good to excellent reproducibility [9]. ICC values between 0.40 and 0.75 are considered to represent fair to good reproducibility whereas values less than 0.40 represent poor reproducibility.

Not many studies have been performed investigating reproducibility of dCA parameters. Studies reporting ICC are performed for spontaneous ABP variations using TFA analysis $[5,11,38]$ and multimodal pressure flow analysis [12] and for paced breathing [11] and sit-stand maneuvers using TFA analysis [38]. Apart from differences in dCA analysis methods also different parameters are reported, which complicates comparison of results between studies. However, a common finding is high reproducibility (ICC $>0.8$ ) for recorded mean finger $\mathrm{ABP}$ and mean $\mathrm{CBFV}$ values using TCD. Only van Beek et al [38] report an ICC of 0.32 for ABP, which is an odd finding. They also report invalid negative ICC for phase in the very low frequency band, which 
make their results dubious. For dCA parameters ICCs are below 0.75 and especially for ARI ICC is very low $(\sim 0.5)$ although these values still are considered to represent fair to good reproducibility [9]. High ICC for mean $\mathrm{ABP}$ and mean CBFV and reduced ICC for dCA parameters deducted from $\mathrm{ABP}$ and $\mathrm{CBFV}$ recordings make clear that either dCA tests or analysis techniques or both need to be improved to increase reproducibility to allow clinical applicability of dCA tests.

Due to low subject numbers between 10 and 20 in studies on reproducibility of dCA parameters confidence intervals for the reported ICC values are very wide. Much larger studies with over 200 subjects on reproducibility are needed to narrow down these confidence intervals of ICC values, but more important is finding ways to improve dCA test reproducibility.

Poor reproducibility of dCA parameters might also be due to true variation of autoregulation. Recent studies in which dCA was evaluated at a one minute time resolution suggest dCA varies over this time range [22, 24, 26]. Further research is needed to investigate what causes the variability of dCA parameters.

\section{Multivariate analysis}

By definition dCA simply involves the univariate relationship between ABP and $\mathrm{CBFV}$, but in practice it is difficult to study this relation without interference of covariates. The influence of covariates may result in decreased coherence. To cope with this, either one has to record under conditions of stable covariates or these covariates need to be monitored and corrected for in the results.

An important covariate is arterial $\mathrm{CO}_{2}$ level, since $\mathrm{CO}_{2}$ as a potent vasodilator largely influences $\mathrm{CBFV}$. In our reproducibility study in chapter 2 we have recorded end-tidal $\mathrm{CO}_{2}$ level $\left(e \mathrm{eCO}_{2}\right)$ in part of the measurements. These recordings showed that et $\mathrm{CO}_{2}$ was decreased in $6 / \mathrm{min}$ breathing compared with normal breathing and dCA phase was increased in these measurements. These findings have also been reported by Reinhard et al using paced breathing [30] and are consistent with our results during $\mathrm{CPB}$ in chapter 6 , where dCA phase decreases with increasing $\mathrm{p}_{\mathrm{a}} \mathrm{CO}_{2}$. This implies $\mathrm{p}_{\mathrm{a}} \mathrm{CO}_{2}$ always needs to be monitored.

Better than monitoring et $\mathrm{CO}_{2}$ and explaining its influence on dCA results, is incorporating recorded etCO $\mathrm{C}_{2}$ as extra input of the transfer function or mathematical model. Recently, a multivariate autoregressive moving average model has been demonstrated to simultaneously analyze the influence of ABP, 
$\mathrm{CO}_{2}$ and motor stimulation when performing repetitive elbow movements [25]. This method also allows for simultaneous evaluation of dCA and neurovascular coupling (NVC).

In chapter 5 we have demonstrated that interaction of dCA during evaluation of NVC may be investigated using a two input-one output transfer function analysis, with both ABP and neural stimulus as inputs and CBFV as output. NVC parameters were more reliably estimated with the blood pressure corrected model than with the basic NVC model. A different approach from a physiological base is combining a lumped parameter model of the circulation with a set of differential equations for physiological mechanisms underlying both dCA and NVC [33]. Using this approach dCA seems mainly dominated by myogenic regulation, whereas NVC can be explained mainly by neurogenic regulation.

\section{Clinical applications}

From previous paragraphs it is clear that methodological improvements need to be made before dCA testing may become a clinically applicable test in the individual. Nevertheless, many studies have been performed in patient groups. However, due to lack of standardization in TFA analysis and lack of normative values, comparison between different studies is difficult. Therefore, each center needs to have its own control group or perform within-subject comparison only, e.g. by comparing between a baseline situation and a situation of impaired dCA after inhalation of $5 \% \mathrm{CO}_{2}$. Aiming for standardized tests analyzed with standardized software would improve result comparability between centers.

In chapter 3 we have studied dCA before and after acetazolamide (ACZ) infusion in a group of patients with small vessel disease (SVD). ACZ infusion may be used to investigate cerebrovascular reactivity (CVR) but due to its vasodilatory effect also a reduction on dCA performance is expected. We have shown that in SVD patients baseline dCA phase was not reduced as compared to dCA phase in healthy controls (Chapter 2). After ACZ infusion which increased CBFV by $67 \%$, phase was decreased only by $27 \%$ and not reduced to zero phase shift, meaning after ACZ infusion still some autoregulatory capacity remains. This implies CVR testing using ACZ infusion is not determining maximum reactivity since further vasodilation is still possible. In chapter 4 we have shown no difference in dCA parameters between patients with Alzheimer's disease (AD) and subjects with mild cognitive impairment (MCI) compared to healthy elderly. In this study, dCA has been studied using spontaneous blood pressure variations. Claassen et al [6] also 


\section{Chapter 7}

studied $\mathrm{dCA}$ in $\mathrm{AD}$ patients. They found, compared to healthy elderly increased gain in the low frequency band $(0.07-0.2 \mathrm{~Hz})$ while using squatstand maneuvers to evoke ABP variation. These maneuvers might, because of larger $\mathrm{ABP}$ variation, challenge $\mathrm{dCA}$ more and therefore make $\mathrm{dCA}$ evaluation more sensitive to differences in cerebral blood flow control.

\section{Limitations}

Our studies maybe have insufficiently addressed the influence of $\mathrm{CO}_{2}$ on dynamic cerebral autoregulation. Both in the studies in healthy volunteers described in chapter 2 and in the study in patients and healthy elderly in chapter 4 et $\mathrm{CO}_{2}$ measurements have been performed. In chapter 2 etCO $\mathrm{C}_{2}$ have been recorded in nine of 19 subjects both during spontaneous and paced breathing. Especially during paced breathing, where subjects are likely to increase their tidal volumes and expire more $\mathrm{CO}_{2}$, influence of etCO $\mathrm{C}_{2}$ could be seen; et $\mathrm{CO}_{2}$ dropped after the start of paced breathing resulting in lower $\mathrm{CBFV}$. The interaction of this etCO $\mathrm{C}_{2}$ change in relation to measures of $\mathrm{dCA}$ should be further investigated. From the study in chapter 6 we know that with lower $\mathrm{p}_{\mathrm{a}} \mathrm{CO}_{2}$ both dCA gain and phase are increased. Therefore, $\mathrm{p}_{\mathrm{a}} \mathrm{CO}_{2}$ always needs to be monitored when investigating dCA.

\section{Future directions}

From the results in this thesis and the progressing research in the field of dynamic cerebral autoregulation new directions in dCA analysis need to evolve towards multivariate analysis. The complex interaction between arterial blood pressure, $\mathrm{p}_{\mathrm{a}} \mathrm{CO}_{2}$ and metabolic stimuli such as visual stimulation needs to be disentangled by developing new experimental setups that try to control the strength of each individual stimulus and analyze the resulting $\mathrm{CBFV}$ in relation to each of the stimuli.

Unless ABP is constant, the continuous process of dCA is interfering with any other cerebral hemodynamic process studied, e.g. neurovascular coupling. When quantifying NVC, CBFV variation is partly caused by the response to e.g. visual stimuli and partly by the dCA response to ABP variation. In chapter 5 we showed estimated NVC parameters are more reliable when also taking into account the effects of dCA on CBFV. When studying dCA, other processes influencing blood pressure and/or cerebral blood flow might need to be studied.

A process that might need to be investigated in conjunction with dCA quantification is the baroreceptor response. Baroreceptors sense in- and decreases of blood pressure in the aorta and carotid arteries and send a 
corresponding signal to the central nervous system. This results in a reaction of the autonomic nervous system: one of the responses is decreasing respectively increasing heart rate to restore blood pressure. Ogoh et al [19] showed baroreceptor reflex plays an important role in dCA when ABP is rapidly decreased. They showed attenuated dCA after full cardiac autonomic (sympathetic-cholinergic) blockade using the leg cuff method. The exact relationship between baroreceptor reflex and $\mathrm{dCA}$ needs to be further investigated.

Another regulatory mechanism that is underexposed but is important for cerebral metabolism is brain temperature regulation [29]. This could be very interesting to study, especially since there is increased attention in brain cooling after stroke and very little is known about the consequences of brain cooling e.g. for cerebral autoregulation.

\section{Conclusions}

The work in this thesis was aimed at investigating methodology of dCA evaluation in order to improve clinical applicability. From this work the following conclusions can be drawn:

- Reproducibility of dCA evaluation based on either spontaneous blood pressure variations or paced breathing is too low for a reliable clinical test.

- Cerebrovascular reactivity index is higher in Alzheimer's disease compared to healthy elderly, whereas dCA parameters do not differ.

- Quantification of NVC is more reliable when incorporating a dCA model for correction of blood pressure related blood flow velocity variations.

- Generating blood pressure variations by cyclic changes of pump speed in a machine for extra corporeal circulation facilitate studying dCA during cardiac bypass surgery. 


\section{References}

1. Aaslid R, Blaha M, Sviri G, Douville CM, and Newell DW. Asymmetric dynamic cerebral autoregulatory response to cyclic stimuli. Stroke 38: 1465-1469, 2007.

2. Aaslid R, and Brubakk AO. Accuracy of an ultrasound Doppler servo method for noninvasive determination of instantaneous and mean arterial blood pressure. Circulation 64: 753-759, 1981.

3. Aaslid R, Lindegaard KF, Sorteberg W, and Nornes H. Cerebral autoregulation dynamics in humans. Stroke 20: 45-52, 1989.

4. Birch AA, Dirnhuber MJ, Hartley-Davies R, Iannotti F, and NeilDwyer $\mathrm{G}$. Assessment of autoregulation by means of periodic changes in blood pressure. Stroke 26: 834-837, 1995.

5. Brodie FG, Atkins ER, Robinson TG, and Panerai RB. Reliability of dynamic cerebral autoregulation measurement using spontaneous fluctuations in blood pressure. Clin Sci (Lond) 116: 513-520, 2009.

6. Claassen JA, Diaz-Arrastia R, Martin-Cook K, Levine BD, and Zhang R. Altered cerebral hemodynamics in early Alzheimer disease: a pilot study using transcranial Doppler. J Alzheimers Dis 17: 621-629, 2009.

7. Claassen JA, Levine BD, and Zhang R. Dynamic cerebral autoregulation during repeated squat-stand maneuvers. J Appl Physiol 106: 153-160, 2009.

8. Diehl RR, Linden D, Lucke D, and Berlit P. Phase relationship between cerebral blood flow velocity and blood pressure. A clinical test of autoregulation. Stroke 26:1801-1804,1995.

9. Fleiss JL. The Design and Analysis of Clinical Experiments. New York: John Wiley, 1986.

10. Giller CA. The frequency-dependent behavior of cerebral autoregulation. Neurosurgery 27: 362-368, 1990.

11. Gommer E, Shijaku E, Mess W, and Reulen J. Dynamic cerebral autoregulation: different signal processing methods without influence on results and reproducibility. Med Biol Eng Comput 1-8, 2010.

12. Hu K, Peng CK, Czosnyka M, Zhao P, and Novak V. Nonlinear assessment of cerebral autoregulation from spontaneous blood pressure and cerebral blood flow fluctuations. Cardiovasc Eng 8: 6071, 2008.

13. Kashif FM, Verghese GC, Novak V, Czosnyka M, and Heldt T. Model-Based Noninvasive Estimation of Intracranial Pressure from 
Cerebral Blood Flow Velocity and Arterial Pressure. Science Translational Medicine 4: 129ra144, 2012.

14. Katsogridakis E, Bush G, Fan L, Birch AA, Simpson DM, Allen R, Potter JF, and Panerai RB. Random perturbations of arterial blood pressure for the assessment of dynamic cerebral autoregulation. Physiological Measurement 33: 103, 2012.

15. Kouchakpour H, Allen R, and Simpson DM. Nonlinear, multipleinput modeling of cerebral autoregulation using Volterra Kernel estimation. In: Engineering in Medicine and Biology Society (EMBC), 2010 Annual International Conference of the IEEE2010, p. 2375-2378.

16. Marmarelis V, Shin D, and Zhang R. Linear and nonlinear modeling of cerebral flow autoregulation using principal dynamic modes. Open Biomed Eng J 6: 42-55, 2012.

17. Marmarelis VZ. Identification of nonlinear biological systems using Laguerre expansions of kernels. Ann Biomed Eng 21: 573-589, 1993.

18. Mitsis GD, Poulin MJ, Robbins PA, and Marmarelis VZ. Nonlinear modeling of the dynamic effects of arterial pressure and $\mathrm{CO} 2$ variations on cerebral blood flow in healthy humans. IEEE Trans Biomed Eng 51: 1932-1943, 2004.

19. Ogoh S, Tzeng YC, Lucas SJ, Galvin SD, and Ainslie PN. Influence of baroreflex-mediated tachycardia on the regulation of dynamic cerebral perfusion during acute hypotension in humans. J Physiol 2009.

20. Panerai RB. Cerebral autoregulation: from models to clinical applications. Cardiovasc Eng 8: 42-59, 2008.

21. Panerai RB, Dawson SL, and Potter JF. Linear and nonlinear analysis of human dynamic cerebral autoregulation. Am J Physiol 277: H10891099, 1999.

22. Panerai RB, Dineen NE, Brodie FG, and Robinson TG. Spontaneous fluctuations in cerebral blood flow regulation: contribution of PaCO2.J Appl Physiol japplphysiol.00857.02010, 2010.

23. Panerai RB, Eames PJ, and Potter JF. Multiple coherence of cerebral blood flow velocity in humans. Am J Physiol Heart Circ Physiol 291: H251-H259, 2006.

24. Panerai RB, Eames PJ, and Potter JF. Variability of time-domain indices of dynamic cerebral autoregulation. Physiol Meas 24: 367-381, 2003.

25. Panerai RB, Salinet ASM, and Robinson TG. Contribution of arterial blood pressure and $\mathrm{PaCO} 2$ to the cerebrovascular responses to motor 
stimulation. American Journal of Physiology - Heart and Circulatory Physiology 302: H459-H466, 2012.

26. Panerai RB, Sammons EL, Smith SM, Rathbone WE, Bentley S, Potter JF, and Samani NJ. Continuous estimates of dynamic cerebral autoregulation: influence of non-invasive arterial blood pressure measurements. Physiol Meas 29: 497-513, 2008.

27. Paulson OB, Strandgaard S, and Edvinsson L. Cerebral autoregulation. Cerebrovasc Brain Metab Rev 2: 161-192, 1990.

28. Raboel PH, Bartek J, Jr., Andresen M, Bellander BM, and Romner B. Intracranial Pressure Monitoring: Invasive versus Non-Invasive Methods-A Review. Crit Care Res Pract 2012: 950393, 2012.

29. Rango M, Arighi A, and Bresolin N. Brain temperature: What do we know? Neuroreport 23: 483-487, 2012.

30. Reinhard M, Muller T, Guschlbauer B, Timmer J, and Hetzel A. Transfer function analysis for clinical evaluation of dynamic cerebral autoregulation - a comparison between spontaneous and respiratoryinduced oscillations. Physiol Meas 24: 27-43, 2003.

31. Schmidt B, Czosnyka M, Raabe A, Yahya H, Schwarze JJ, Sackerer D, Sander D, and Klingelhofer J. Adaptive noninvasive assessment of intracranial pressure and cerebral autoregulation. Stroke 34: 84-89, 2003.

32. Schmidt B, Schwarze JJ, Czosnyka M, Sander D, Wittich I, and Klingelhofer J. A method for a simulation of continuous intracranial pressure curves. Comput Biomed Res 31: 231-243, 1998.

33. Spronck B, Martens EG, Gommer ED, and van de Vosse FN. A lumped parameter model of cerebral blood flow control combining cerebral autoregulation and neurovascular coupling. Am J Physiol Heart Circ Physiol 303: H1143-1153, 2012.

34. Stanforth PR, Gagnon J, Rice T, Bouchard C, Leon AS, Rao DC, Skinner JS, and Wilmore JH. Reproducibility of Resting Blood Pressure and Heart Rate Measurements: The HERITAGE Family Study. Annals of Epidemiology 10: 271-277, 2000.

35. Tarvainen MP, Ranta-Aho PO, and Karjalainen PA. An advanced detrending method with application to HRV analysis. IEEE Trans Biomed Eng 49: 172-175, 2002.

36. Tiecks FP, Lam AM, Aaslid R, and Newell DW. Comparison of static and dynamic cerebral autoregulation measurements. Stroke 26: 10141019, 1995. 
37. Timmer J, Lauk M, and Deuschl G. Quantitative analysis of tremor time series. Electroencephalogr Clin Neurophysiol 101: 461-468, 1996.

38. van Beek AH, Olde Rikkert MG, Pasman JW, Hopman MT, and Claassen JA. Dynamic Cerebral Autoregulation in the Old Using a Repeated Sit-Stand Maneuver. Ultrasound Med Biol 36: 192-201, 2010.

39. Welch PD. The use of fast Fourier transform for the estimation of power spectra: a method based on time averaging over short, modified periodograms. IEEE Trans Audio Electroacoust 15: 70-73, 1967.

40. Zhang R, Claassen JA, Shibata S, Kilic S, Martin-Cook K, DiazArrastia R, and Levine BD. Arterial-cardiac baroreflex function: insights from repeated squat-stand maneuvers. Am J Physiol Regul Integr Comp Physiol 297: R116-123, 2009. 

Summary 


\section{Summary}

Maintenance of adequate cerebral blood flow is essential for normal brain function and survival. The brain receives approximately $15 \%$ of cardiac output and is responsible for approximately $20 \%$ of total body oxygen consumption. Because of the brain's limited ability to store energy cerebral blood flow needs to be controlled effectively. To adjust cerebral blood flow to an adequate level the diameter of cerebral vessels is changed. Cerebral blood flow (CBF) is dynamically adjusted to changes in the perfusion pressure, the metabolic activity of the brain, humoral factors and autonomic nerve activity.

Control of cerebral blood flow to adjust for changes in cerebral perfusion pressure is called cerebral autoregulation. Disease states of the brain may impair or abolish cerebral autoregulation. The exact function of cerebral autoregulation still remains unclear. Three different mechanisms are thought to play a role contributing to cerebral autoregulation: metabolic, myogenic and neurogenic regulation.

Being able to reliably estimate parameters of dynamic cerebral autoregulation with sufficient reproducibility by non-invasive means could improve patient diagnostics and management for several conditions. To assess cerebral autoregulation at least recording of blood pressure and a measure for $\mathrm{CBF}$ are required. This thesis aims to improve clinical applicability of testing dynamic cerebral autoregulation (dCA). The first aim of this thesis is to evaluate the use of transfer function analysis (TFA) for quantifying dynamic cerebral autoregulation. Secondly our aim is to evaluate clinical application of the developed dCA analysis.

In chapter 2 dCA reproducibility, quantified by intraclass correlation coefficient, is evaluated for different methodological approaches of TFA. Analysis compared raw data pre-processing by mean subtraction versus smoothness priors detrending. Furthermore, spectral density estimation by averaging of subsequent time windows versus smoothing the whole recording spectrum. No significant influence of pre-processing and spectral estimation on dCA parameters was found. Therefore, there seems to be no need to prescribe a specific signal-processing regime. Poor reproducibility of gain and phase was found. Based on reproducibility, no preference can be made for morning versus afternoon measurements. Neither for spontaneous versus paced breathing.

In chapter 3 is evaluated if, and to what degree dCA was changed after acetazolamide (ACZ) infusion. Using a body weight adjusted ACZ infusion 
cerebrovascular reactivity (CVR) was assessed in 29 first ever lacunar stroke patients. After ACZ infusion, median phase angle decreased significantly ( $\mathrm{p}<0.005$ Wilcoxon) to $0.77 \mathrm{rad}$ compared to a pre-test baseline value of 1.05 rad, indicating less efficient dCA due to ACZ. However, post-test phase values are still mostly within the normal range. It can be concluded that CVR testing with body weight adjusted infusion of ACZ lowers dCA performance but by no means exhausts $\mathrm{dCA}$, suggesting that in this way maximal CVR is not determined.

Cerebrovascular dysfunction plays a role not only in vascular causes of cognitive impairment but also in Alzheimer's disease (AD). In chapter 4 we hypothesized that dynamic cerebral autoregulation is impaired in patients with $\mathrm{AD}$ compared to subjects with mild cognitive impairment (MCI) and controls (C). Apart from measures of $\mathrm{dCA}$ also cerebrovascular resistance index CVRi was computed. dCA in supine position was quantified based on spontaneous blood pressure variations by computation of the linear transfer function between arterial blood pressure and MCA cerebral blood flow velocity. Results were also evaluated using a 3-parameter windkessel model (WKM).

CVRi was significantly higher in $\mathrm{AD}$ compared to both $\mathrm{MCI}$ and C. Five MCI patients who converted to AD during the course of the study also had higher CVRi compared to non-converters. No significant differences in dCA gain and phase were found. In terms of the WKM approach, in the order $\mathrm{C} \rightarrow \mathrm{MCI} \rightarrow \mathrm{AD}$ groups showed about equal arterial resistance and peripheral compliance, but increased peripheral vasculature resistance. In AD patients compared to MCI patients and controls we found increased CVRi, whereas dCA parameters do not seem to differentiate AD patients. For MCI patients CVRi might have predictive value in developing AD.

Chapter 5 describes how parameter estimation of visually evoked flow responses (VEFR) may be improved when the effect of blood variations through dCA are incorporated in the model. VEFR are often quantified using a dynamic model of neurovascular coupling (NVC). The VEFR is seen as the model's response to a visual step input stimulus. However, the continuously active process of dynamic cerebral autoregulation (dCA) compensating cerebral blood flow for blood pressure fluctuations may induce changes of cerebral blood flow velocity (CBFV) as well. The effect of blood pressure variability on VEFR is evaluated by separately modeling the dCA induced effects of beat-to-beat measured blood pressure related CBFV changes.

VEFR NVC parameters of 71 subjects are estimated using two models: the RG model is a well-known second order dynamic NVC model. The second $\mathrm{RG}_{\mathrm{CA}}$ 
model extends the RG model with a CBFV contributing component being the output of a dCA model driven by blood pressure as input.

Both models were evaluated for mean and systolic CBFV VEFR responses. The model-to-data fit errors from the $\mathrm{RG}_{\mathrm{CA}}$ mean and systolic blood pressure corrected model were significantly lower compared to the RG model: mean $0.8 \% \pm 0.6$ vs. $2.4 \% \pm 2.8 \mathrm{p}<0.001$ systolic $1.5 \% \pm 1.2$ vs. $2.2 \% \pm 2.6 \mathrm{p}<0.001$. The confidence bounds of all $\mathrm{RG}_{\mathrm{CA}}$ estimated NVC model parameters were significantly $(\mathrm{p}<0.005)$ narrowed.

In conclusion, blood pressure correction of NVC responses by including cerebral autoregulation in model fitting of averaged VEFR responses results in significantly lower fit errors and by that in more reliable model parameter estimation. Blood pressure correction is more effective when mean instead of systolic CBFV responses are used. Measurement and quantification of NVC should include beat-to-beat blood pressure measurement.

In chapter 6 a technique is introduced to evaluate dCA during normothermic nonpulsatile cardiopulmonary bypass (CPB). The technique uses continuous recording of invasive arterial blood pressure, middle cerebral artery blood flow velocity through transcranial Doppler sonography, in-line arterial carbon dioxide and pump flow measurement. dCA during CPB is estimated by TFA based on the response to blood pressure variation induced by cyclic 6/min changes of indexed pump flow from 2.0 to 2.4 up to $2.8 \mathrm{l} / \mathrm{min} / \mathrm{m} 2$. In 37 patients during $\mathrm{CPB}$ we tested the feasibility of our technique to estimate $\mathrm{dCA}$ at hypocapnia $\left(\mathrm{p}_{\mathrm{a}} \mathrm{CO}_{2}=30 \mathrm{mmHg}\right)$, normocapnia $\left(\mathrm{p}_{\mathrm{a}} \mathrm{CO}_{2}=40 \mathrm{mmHg}\right)$, and hypercapnia $\left(\mathrm{p}_{\mathrm{a}} \mathrm{CO}_{2}=50 \mathrm{mmHg}\right)$. dCA phase decreased significantly $(\mathrm{p}<0.01)$ with increasing $\mathrm{p}_{\mathrm{a}} \mathrm{CO}_{2}$ from hypocapnia $(0.58 \pm 0.3 \mathrm{rad})$ to normocapnia $(0.31 \pm 0.2 \mathrm{rad})$ and hypercapnia $(0.1 \pm 0.1 \mathrm{rad})$. Also gain decreased $(\mathrm{p}<0.01)$ with increasing $\mathrm{p}_{\mathrm{a}} \mathrm{CO}_{2}$ from hypocapnia $(3.4 \pm 2 \% / \mathrm{mmHg})$ to normocapnia $(2.2 \pm 0.9 \% / \mathrm{mmHg})$ and hypercapnia $(1.4 \pm 0.4 \% / \mathrm{mmHg})$. Studying dynamic cerebral autoregulation during cardiopulmonary bypass is feasible by changing pump flow in a $6 / \mathrm{min}$ rate. Hypercapnia results in impaired dCA. Ongoing and future studies are expected to clarify optimal perfusion strategies for various patient populations scheduled for operations involving cardiopulmonary bypass.

In chapter 7 the main findings and shortcomings of this thesis are discussed as well as their implications for future research. 
Samenvatting 


\section{Samenvatting}

Voor de normale hersenfunctie en in extremis voor overleving is een adequate handhaving van de cerebrale bloedstroom van essentieel belang. Ongeveer $15 \%$ van de cardiale uitstroom gaat naar het brein, dat ongeveer $20 \%$ van het totale lichaamszuurstofverbruik voor zijn rekening neemt. Vanwege de beperkte mogelijkheden van het brein voor energieopslag is het nodig de cerebrale doorbloeding goed te reguleren. Het aanpassen van de cerebrale bloedstroom gebeurt door de doorsnede van de cerebrale vaten te veranderen. De cerebrale bloedstroom wordt dynamisch aangepast aan veranderingen in de perfusiedruk, de metabole activiteit van het brein, hormonale factoren en autonome zenuwactiviteit.

De regulering van de cerebrale bloedstroom voor veranderingen in de perfusiedruk wordt cerebrale autoregulatie genoemd. Bij verschillende ziektebeelden kan de functie van cerebrale autoregulatie verstoord of zelfs afwezig zijn. De exacte werking van cerebrale autoregulatie is nog slecht begrepen. De volgende drie verschillende mechanismen worden gedacht een rol te spelen: metabole, myogene and neurogene regulatie.

Het betrouwbaar, voldoende reproduceerbaar en niet-invasief kunnen meten van dynamische cerebrale autoregulatie (dCA) kan de diagnostiek en behandeling van meerdere ziektebeelden verbeteren. Minimaal is het hiervoor nodig continu de bloeddruk en een maat voor de cerebrale bloedstroom te meten. Dit proefschrift heeft als doel de klinische toepasbaarheid te verbeteren van dynamische cerebrale autoregulatietesten. Als eerste wordt het gebruik van de methode op basis van overdrachtsfunctieanalyse (OFA) onderzocht. Ten tweede wordt de klinische toepassing van de ontwikkelde methode getest in diverse patiëntengroepen.

In hoofdstuk 2 is de reproduceerbaarheid van dCA onderzocht voor verschillende OFA parameterkeuzes, gekwantificeerd met de intraklasse correlatie coëfficiënt. Twee verschillende data voorbewerkingstappen worden vergeleken, namelijk het verwijderen van de offset door de gemiddelde waarde af te trekken en een complexere methode genaamd smoothness priors detrending. Daarnaast wordt de schatting van de spectrale dichtheid bepaald op twee manieren, namelijk door middeling van opeenvolgende segmenten en door het gladder maken van het spectrum. Er worden geen significante invloeden gevonden voor de voorbewerkingstappen noch voor de methode van spectrale schatting. Dit maakt dat er geen noodzaak lijkt voor het voorschrijven van een specifiek signaalbewerkingsregime. Voor zowel fase als gain wordt een matige reproduceerbaarheid gevonden. Op basis van de 
reproduceerbaarheid kan geen voorkeur worden bepaald voor het meten in de ochtend of de middag noch voor spontane versus opgelegde ademhaling. In hoofdstuk 3 is onderzocht of, en in welke mate dCA verandert na infusie van acetazolamide (ACZ). Met een lichaamsgewichtgecorrigeerde dosis van 15 $\mathrm{mg} / \mathrm{kg} \mathrm{ACZ}$ wordt de cerebrovasculaire reactiviteit (CVR) getest in 29 eerst optredende lacunaire stroke patiënten. Na ACZ infusie, neemt de mediaan van de fasehoek significant ( $p<0.005$ Wilcoxon) af tot 0.77 rad vergeleken met een uitgangswaarde van $1.05 \mathrm{rad}$, wat duidt op een minder efficiënte dCA ten gevolge van ACZ. Echter, de fasewaarden zijn na infusie nog grotendeels in het normale bereik. Derhalve kan worden geconcludeerd dat het testen van CVR met een ACZ dCA prestaties vermindert maar niet leidt tot uitschakeling van het regelmechanisme, wat suggereert dat niet de maximale CVR is bepaald.

Verminderde functie van de cerebrale vaten speelt niet alleen een rol in de oorzaak van vermindering van de cognitieve functie, maar ook in de ziekte van Alzheimer (AZ). In hoofdstuk 4 wordt verondersteld dat dynamische cerebrale autoregulatie functie verminderd is in patiënten met $A Z$ vergeleken met ouderen met mild cognitive impairment (MCI) en een gezonde oudere controlegroep (C). Naast de maten voor dCA wordt ook de cerebrovasculaire weerstandsindex CVWi berekend. dCA wordt in liggende positie gekwantificeerd op basis van spontane bloeddrukvariaties door berekening van de lineaire overdrachtsfunctie tussen arteriële bloeddruk en cerebrale bloedstroomsnelheid in de middelste cerebrale arterie (MCA). De resultaten worden tevens gekwantificeerd door middel van een 3-parameter windketel model (WKM).

CVWi is significant hoger in AZ vergeleken met zowel MCI als C. Vijf MCI patiënten die gedurende de studie converteerden naar $\mathrm{AZ}$ hebben ook een hogere CVWi vergeleken met de niet-geconverteerde. Er worden geen significante verschillen gevonden in dCA gain and fase. Voor het WKM wordt in de volgorde $\mathrm{C} \rightarrow \mathrm{MCI} \rightarrow \mathrm{AZ}$ een ongeveer gelijke arteriële weerstand en perifere compliantie gevonden, maar een verhoogde perifere vaatweerstand. In $\mathrm{AZ}$ patiënten wordt vergeleken met $\mathrm{MCI}$ patiënten en controles een verhoogde CVWi gevonden, terwijl op basis van dCA parameters AZ patiënten niet kunnen worden onderscheiden. Voor MCI patiënten zou een verhoogde CVWi een voorspellende waarde kunnen hebben voor het ontwikkelen van AZ.

Hoofdstuk 5 laat zien dat parameterschatting van visueel opgewekte flow responsies (VOFR) verbeterd kan worden door in het model te corrigeren voor de effecten van bloeddrukvariaties via dCA. VOFR worden doorgaans 
gekwantificeerd door middel van een dynamisch model van neurovasculaire koppeling (NVK). De VOFR wordt beschouwd als het antwoord van het model op een stapvormige visuele stimulus. De gemeten flow bestaat echter ook uit een component die ontstaat door de reactie van het continu actieve proces van dCA op optredende bloeddrukvariaties. Dit effect wordt geëvalueerd door het NVK-model uit te breiden met een model voor dCA.

VOFR NVC parameters van 71 patiënten zijn geschat met twee modellen: het $R G$ model is het bekende $2^{\mathrm{e}}$ orde dynamische $\mathrm{NVC}$ model. Het tweede $\mathrm{RG}_{\mathrm{CA}}$ model is een uitbreiding van het RG model met een CBFV component die de uitgang is van een dCA model met bloeddruk als ingang.

Beide modellen worden geëvalueerd voor gemiddelde en systole CBFV VOFR responsies. De model-naar-data fitfouten zijn voor het $\mathrm{RG}_{\mathrm{CA}}$ gemiddelde en systole bloeddrukgecorrigeerde model significant lager vergeleken met het RG model: gemiddelde $0.8 \% \pm 0.6$ vs. $2.4 \% \pm 2.8 \mathrm{p}<0.001$, systole $1.5 \% \pm 1.2$ vs. $2.2 \% \pm 2.6 \mathrm{p}<0.001$. De betrouwbaarheidsintervallen van alle $R_{C A}$ geschatte NVC model parameters zijn significant $(\mathrm{p}<0.005)$ smaller.

Geconcludeerd kan worden dat bloeddrukcorrectie bij het fitten van NVC responsies, door dCA toe te voegen aan het model, leidt tot significant lagere fitfouten en tot betrouwbaardere schattingen van de modelparameters. Bloeddrukcorrectie blijkt effectiever wanneer gemiddelde in plaats van systole CBFV responsies worden gebruikt. Bij meting en kwantificering van NVC dient daarom slag-op-slag bloeddrukmeting plaats te vinden.

In hoofdstuk 6 wordt een methode geïntroduceerd om dCA te evalueren tijdens normothermische nonpulsatiele cardiopulmonaire bypasschirurgie (CPB). Deze methode berust op continue meting van invasieve arteriële bloeddruk, MCA bloedstroomsnelheid door transcraniele Doppler sonografie, in-lijn arteriële $\mathrm{CO}_{2}$ concentratie en pompflow. dCA wordt tijdens $\mathrm{CPB}$ bepaald door middel van TFA, gebaseerd op geïnduceerde bloeddrukvariatie door cyclische $6 / \mathrm{min}$ veranderingen van pompflow van 2.0 naar 2.4 tot 2.8 $1 / \mathrm{min} / \mathrm{m} 2$. In 37 patiënten wordt tijdens CPB de haalbaarheid getest van deze methode om dCA te evalueren bij hypocapnie $\left(\mathrm{p}_{\mathrm{a}} \mathrm{CO}_{2}=30 \mathrm{mmHg}\right)$, normocapnie $\left(\mathrm{p}_{\mathrm{a}} \mathrm{CO}_{2}=40 \mathrm{mmHg}\right)$ en hypercapnie $\left(\mathrm{p}_{\mathrm{a}} \mathrm{CO}_{2}=50 \mathrm{mmHg}\right)$. $\mathrm{dCA}$ fase neemt significant af $(\mathrm{p}<0.01)$ bij toename van $\mathrm{p}_{\mathrm{a}} \mathrm{CO}_{2}$ van hypocapnie $(0.58$ $\pm 0.3 \mathrm{rad})$ naar normocapnie $(0.31 \pm 0.2 \mathrm{rad})$ en hypercapnie $(0.1 \pm 0.1 \mathrm{rad})$. Ook de gain neemt af $(\mathrm{p}<0.01)$ bij toegenomen $\mathrm{p}_{\mathrm{a}} \mathrm{CO}_{2}$ van hypocapnie $(3.4 \pm 2$ $\% / \mathrm{mmHg})$ naar normocapnie $(2.2 \pm 0.9 \% / \mathrm{mmHg})$ en hypercapnie $(1.4 \pm 0.4$ $\% / \mathrm{mmHg}$ ). Geconcludeerd kan worden dat evaluatie van dCA tijdens CPB mogelijk is door het veranderen van de pompflow in een ritme van $6 / \mathrm{min}$. Hypercapnie resulteert in verminderde dCA. Verdere studies kunnen 


\section{Samenvatting}

mogelijk leiden tot optimale perfusiestrategiën bij procedures waarbij bypasschirurgie nodig is.

In hoofdstuk 7 worden de belangrijkste bevindingen en tekortkomingen van dit proefschrift en hun betekenis voor toekomstig onderzoek besproken. 

Dankwoord 


\section{Dankwoord}

Oorspronkelijk was mijn plan om het dankwoord op te zetten met analogieën verwijzend naar de wielersport, waarbij de individuele prestatie alleen tot stand kan komen door goed teamwerk en waarbij verschillende rollen zijn weggelegd voor de teammanager, ploegleider, trainers, coaches, knechten en waterdragers. Gezien de huidige crisis waarin de wielersport zich bevindt en de slechte associaties die dit mogelijk zou oproepen heb ik daar toch maar van afgezien.

Afzien is trouwens wel een woord dat van toepassing is op mijn en misschien wel elk promotietraject, maar wanneer het doel dan eindelijk is bereikt vergeet je dat al weer gauw en besef je dat je veel mensen dank verschuldigd bent die belangrijk zijn geweest om dit traject tot een goed einde te brengen.

Allereerst, prof. dr. Mess, beste Werner, wil ik jou als $1^{\mathrm{e}}$ promotor bedanken voor het geven van de mogelijkheid om te promoveren binnen jouw afdeling Klinische Neurofysiologie. Je aanstekelijke enthousiasme voor de toepassing van ultrageluid binnen de neurologie heeft ook mij geraakt. Je creatieve ideeën hebben tot verrassende methoden geleid. Het is prettig om met je samen te werken.

Professor Panerai PhD, dear Ronney, I am very grateful for your contribution as $2^{\text {nd }}$ promoter of my thesis project. With your huge expertise in the field of cerebral autoregulation you helped me a lot and your critical notes and suggestions on all my papers have been extremely valuable. My visits to Leicester always gave me a big motivational boost. Maybe I should have come more often. All the best for you and Ivone and I hope you will enjoy your future life after you are retired now.

Dr. ir. Reulen, beste Jos, een copromotor levert traditioneel een grote bijdrage aan het promotiewerk. Dat geldt zeker ook voor jou. Het cerebrovasculair onderzoek was bovendien ook voor jou een redelijk nieuw terrein, waarin jij je net als ik hebt moeten inwerken. Jouw succesvolle inspanning om onderzoeksgeld te verwerven bij de Alzheimerstichting was zeer belangrijk voor het onderzoek. Je vele kritische vragen en consciëntieuze correcties op de artikelen hebben dit boekje zeker beter gemaakt. Dank voor alle ondersteuning in de afgelopen jaren. 
Dr. ir. Martens, beste Esther, we zijn jaren samen opgetrokken in het cerebrovasculaire onderzoek, jij op het gebied van de neurovasculaire koppeling en ik op het gebied van de cerebrale autoregulatie. We hebben ooit gefilosofeerd over een duo-promotie, maar dat was toch lastig op twee universiteiten en je was me ruim te snel af. Dank voor het werk dat we samen tot een goed einde hebben gebracht en succes met je opleiding tot klinisch fysicus.

Prof. dr. van Oostenbrugge, beste Robert, in het begin van het onderzoekstraject, toen je nog geen hoogleraar en afdelingshoofd was, hebben we vele discussies gehad over het meten van dynamische cerebrale autoregulatie bij patiënten met lacunaire infarcten, sub-arachnoïdale bloedingen, etc.. Je bent altijd erg geïnteresseerd en bent waarschijnlijk degene die me het vaakst gevraagd heeft hoe het met mijn boekje ging. Je enthousiasme heeft me zeker geholpen.

Dr. Staals, beste Julie, je hebt laten zien een dokter en onderzoeker te zijn die niet aarzelt om zelf de naald in je te laten steken. Je wilde zelf ervaren wat acetazolamide met je doet en hoe zo'n onderzoek in zijn werk gaat. Dank voor de studie die we samen hebben kunnen doen.

Prof. dr. Verhey, dr. Aalten, dr. Ramakers, beste Frans, Paulien en Inez, dankzij jullie hebben we samen een mooi onderzoek kunnen doen in patiënten met de ziekte van Alzheimer en MCI. Hopelijk leidt dit nog tot meer mooie publicaties.

Beste Ervin, dat onderzoek moeilijk te plannen is, is wel gebleken uit de tijd die nodig bleek om de metingen voor hoofdstuk 5 te verzamelen, maar de eerste resultaten zijn hoopgevend. Ik wens je veel succes met het afronden van jouw proefschrift.

Bedankt Boris, Eri, Guy, Bart, Vera, Jochem, Arthur, Anne. Jullie hebben als afstudeer- en stagestudenten meegewerkt aan het onderzoek. Jullie hebben allemaal een belangrijke bijdrage geleverd aan deelaspecten, ook al is jullie werk niet allemaal in dit boekje opgenomen.

Prof. dr. ir. van de Vosse, beste Frans, bedankt voor je bijdrage als voorzitter van de beoordelingscommissie. Hopelijk kunnen we de samenwerking op gebied van modellen van cerebrale autoregulatie voortzetten. De overige 
leden van de beoordelingscommissie, dr. J. Claassens, prof. dr. J.G. van Dijk, prof. dr. H. Kingma en prof. dr. M. Post, wil ik bedanken voor de inspanning om mijn proefschrift te lezen en te beoordelen.

Dank aan alle patiënten en gezonde vrijwilligers die aan de diverse onderzoeken hebben meegedaan.

Dr. ir. van Waalwijk van Doorn, beste Ernst, als mijn opleider heb je me ook voor onderzoek enthousiast gemaakt. We hadden destijds grote plannen. Dit is door allerlei omstandigheden anders gelopen.

Beste Jeroen Hopman, het is er dan toch van gekomen. Bij jou heb ik als eerste kennis gemaakt met het vak van klinisch fysicus, waarvoor ik uiteindelijk in Maastricht ben beland. Onze onderzoeksinspanningen, nu zo'n 20 jaar geleden, om tot automatische analyse van het neonatale EEG te komen blijken nog steeds actueel en houden ook mij weer bezig.

Beste Pim, we samen al heel wat rondjes rond het MUMC, of mogen het (nog) niet zo noemen, gelopen. We hebben daarbij al heel wat balletjes opgeworpen en veel besproken over de toestand in de wereld of wetenschappelijke zaken. Daar gaan we nog wel even mee door. We moeten toch ooit een keer nul fouten kunnen halen in de wetenschapsquiz.

De sfeer op de afdeling Klinische Neurofysiologie is altijd heel prettig en dat komt niet in de laatste plaats door de gezellige groep laboranten en secretaresses. Alle (ex-)collega's bedankt daarvoor. Het regelmatig eten van een stuk taart maakt dit allemaal nog aangenamer. Als de morellenoogst dit jaar weer goed is neem ik weer een taart mee.

Beste Vetmannen, ik heb het afgelopen jaar een beetje teveel laten afweten, maar dat ga ik nu weer goedmaken. Dit wordt weer een mooi wielerjaar, met zo nu en dan een leuk verzetje.

Beste Toussaint en Stefan, bedankt dat jullie mij terzijde willen staan als mijn paranimfen.

Lieve pa en ma, hoe ver ik ook van huis ben afgedreven, mijn wortels liggen nog altijd bij jullie. Jullie hebben mij mede gemaakt tot wat ik nu ben. Bedankt voor jullie onvoorwaardelijke steun en interesse. 
Lieve Bram en Esther, jullie zijn absoluut mijn grootste motivatie om dit boekje uiteindelijk af te maken. Ik heb nu weer meer tijd om leuke dingen met jullie te doen.

Lieve Anke, we hebben het ons de laatste jaren niet makkelijk gemaakt. Jij hebt een opleiding Veiligheidskunde gedaan die je uiteindelijk moest afronden naast een drukke nieuwe baan. Mijn promotie liet maar op zich wachten. Vooral dankzij jouw harde werk en het draaiende houden van ons gezin staan we waar we nu staan en mogen we dat eindelijk samen vieren. Laten we nog lang van het Limburgse leven genieten. 



\section{List of publications}

Gommer E, Shijaku E, Mess W, and Reulen J. Dynamic cerebral autoregulation: different signal processing methods without influence on results and reproducibility. Med Biol Eng Comput 1-8, 2010.

Gommer ED, Martens EG, Aalten P, Shijaku E, Verhey FR, Mess WH, Ramakers IH, and Reulen JP. Dynamic Cerebral Autoregulation in Subjects with Alzheimer's Disease, Mild Cognitive Impairment, and Controls: Evidence for Increased Peripheral Vascular Resistance with Possible Predictive Value. J Alzheimers Dis 30: 805-813, 2012.

Gommer ED, Staals J, van Oostenbrugge RJ, Lodder J, Mess WH, and Reulen JP. Dynamic cerebral autoregulation and cerebrovascular reactivity: a comparative study in lacunar infarct patients. Physiol Meas 29: 1293-1303, 2008 .

Gommer ED, Vanspauwen TJ, Miklosi M, Wen JG, Kinder MV, Janknegt RA, and van Waalwijk van Doorn ES. Validity of a non-invasive determination of the isovolumetric bladder pressure during voiding in men with LUTS. Neurourol Urodyn 18: 477-486, 1999.

Gradaus R, Gonska BD, Stellbrink C, Cron T, Tebbenjohanns J, BlomstromLundqvist C, Himmrich E, Salerno JA, Osswald S, Gommer ED, Van Veen BK, and Bocker D. European clinical experience with a dual chamber single pass sensing and pacing defibrillation lead. Pacing Clin Electrophysiol 25: 1079-1086, 2002.

Kinder M, Gommer E, Janknegt R, and van Waalwijk van Doorn E. Recording the detrusor electromyogram is still a difficult and controversial enterprise. Neurourol Urodyn 17: 571-573, 1998.

Kinder MV, Gommer ED, Janknegt RA, and van Waalwijk van Doorn ES. A method for the electromyographic mapping of the detrusor smooth muscle. Arch Physiol Biochem 105: 673-690, 1997.

Kinder MV, van Waalwijk van Doorn ES, Gommer ED, and Janknegt RA. A non-invasive method for bladder electromyography in humans. Arch Physiol Biochem 106: 2-11, 1998. 
Martens EG, Peeters LL, Gommer ED, Mess WH, van de Vosse FN, Passos VL, and Reulen JP. The visually-evoked cerebral blood flow response in women with a recent history of preeclampsia and/or eclampsia. Ultrasound Med Biol 35: 1-7, 2009.

Meel-van den Abeelen ASS, Lagro J, Gommer ED, Reulen JPH, and Claassen JAHR. Baroreflex function is reduced in Alzheimer's disease: a candidate biomarker? Neurobiol Aging 34: 1170-1176, 2013.

Spronck B, Martens EGHJ, Gommer ED, and van de Vosse FN. A lumped parameter model of cerebral blood flow control combining cerebral autoregulation and neurovascular coupling. Am J Physiol Heart Circy Physiol 303: H1143-H1153, 2012.

van Waalwijk van Doorn ES, and Gommer ED. Ambulatory urodynamics. Curr Opin Obstet Gynecol 7: 378-381, 1995. 


\section{Curriculum Vitae}

Erik Gommer was born on February 10, 1968 in Emmen, the Netherlands. He grew up in Assen were he completed his secondary education (VWO) in 1986 at the Christelijke Scholengemeenschap Assen. In 1992 he graduated in electrical engineering at the Twente university. Additionally, he got a certificate in biomedical engineering. After his study he completed his social service in the pediatrics department of the St. Radboud university hospital performing research on neonatal EEG analysis. In 1994 he started his training as a clinical physicist in the Maastricht university hospital, which he completed in 1998. He then worked for a few years as a scientist at Medtronic Bakken Research Center. In 2001 he returned to the Maastricht university hospital. First as an employee of the Interuniversity Cardiology Institute in the Netherlands and from 2002 as clinical physicist at the biomedical engineering department, where he started his research on cerebral autoregulation. Since 2010 he is clinical physicist at the department of clinical neurophysiology.

Erik is married to Anke de Vries. They have two children: Bram en Esther. 